Portland State University

PDXScholar

$1-1-2010$

\title{
Investigating Freeway Speed-Flow Relationships for Traffic Assignment Applications
}

Meead Saberi Kalaee

Portland State University

Follow this and additional works at: https://pdxscholar.library.pdx.edu/open_access_etds Let us know how access to this document benefits you.

Recommended Citation

Saberi Kalaee, Meead, "Investigating Freeway Speed-Flow Relationships for Traffic Assignment Applications" (2010). Dissertations and Theses. Paper 33.

https://doi.org/10.15760/etd.33

This Thesis is brought to you for free and open access. It has been accepted for inclusion in Dissertations and Theses by an authorized administrator of PDXScholar. Please contact us if we can make this document more accessible: pdxscholar@pdx.edu. 


\title{
Investigating Freeway Speed-Flow Relationships for Traffic Assignment Applications
}

\author{
by \\ Meead Saberi Kalaee
}

\begin{abstract}
A thesis submitted in partial fulfillment of the requirements for the degree of
\end{abstract}

\author{
Master of Science \\ in \\ Civil and Environmental Engineering
}

Thesis Committee:

Miguel A. Figliozzi, Chair

Christopher M. Monsere

John Gliebe

Portland State University

(C)2010 
Abstract

Developments in high resolution traffic sensors over the past decades are providing a wealth of empirical speed-flow data. Travel demand models use speed-flow relationships to assign traffic flows to network links. However, speed-flow relationships have not been revalidated against new detailed traffic sensor data. Therefore, it is necessary to revisit speed-flow relationships based on actual measured conditions on network links rather than assuming constant speed-flow relationships over entire highway network systems.

Speed-flow relationships have been particularly difficult to calibrate and estimate when traffic volumes approach capacity, i.e. when the v/c ratio approaches one. This thesis empirically evaluates the speed-flow relationships for $\mathrm{v} / \mathrm{c}<1$ using field data. For congested conditions (v/c $>1$ ) a theoretical approach is taken. A new methodology to determine the distribution of the activation of bottlenecks, bottleneck duration, and bottleneck deactivation is proposed. This thesis is a new contribution to understand the stochastic nature of freeway capacity as well as bottleneck duration, activation, and deactivation. Unlike previous research efforts, this thesis studies speed-flow 
relationships at the lane level and later presents a method to estimate speedflow relationships at the link level. 


\section{To my parents,}

for your love and support through every joy and hardship

you made me who I am today 


\section{Acknowledgments}

First and foremost I would like to thank God for all the things he has given me and for bringing me to this point in my life.

I want to acknowledge my great family who has always supported me.

I express my infinite love to my parents and my brother and his wife.

I owe so much to my former advisor Robert Bertini. His example is one I will always like to follow. I am also grateful to my thesis advisor Miguel Figliozzi because of his patience, support, and invaluable advice throughout the process of completing this work. I want to acknowledge Christopher Monsere and John Gliebe who have been an inspiration for me in their teaching and their knowledge and for serving on my committee.

I am full of gratitude to the Department of Civil and Environmental Engineering and OTREC staff who have always been there for me.

Finally I want to acknowledge all my friends in the ITS Lab who have helped me in so many ways while I have been at Portland State University. Especially Alex Bigazzi, Sirisha Kothuri, Kaveh Shabani, and Heba Alwakiel who spent hours reviewing this thesis. I am also thankful to Somaye Asadi, Shahrbanou Madadgar, and Kouros Monsef for their support and friendship. 


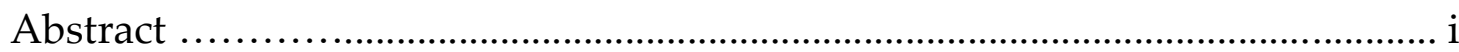

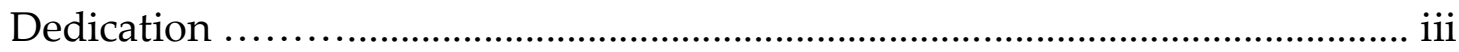

Acknowledgments ............................................................................................. iv

List of Tables .................................................................................................... vii

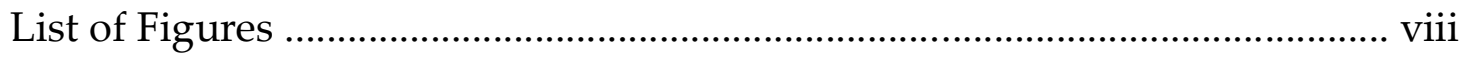

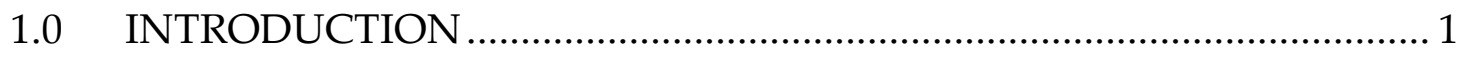

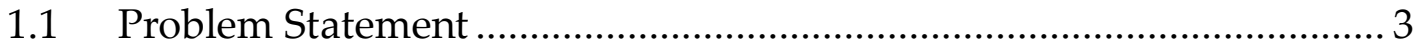

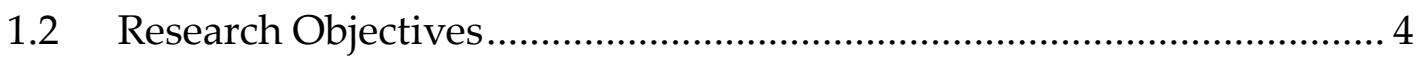

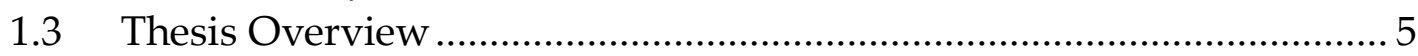

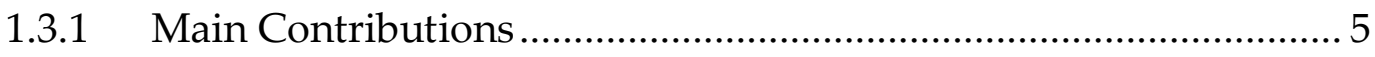

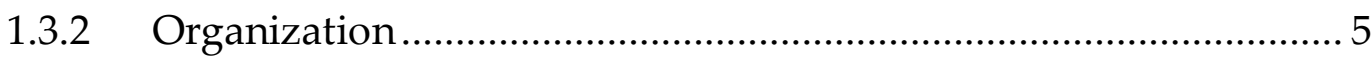

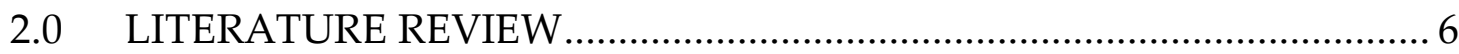

$2.1 \quad$ Traffic Assignment ............................................................................... 6

2.2 Speed-Flow Relationships for Planning Applications ………................ 8

2.3 Calibration of Speed-Flow Models ......................................................... 18

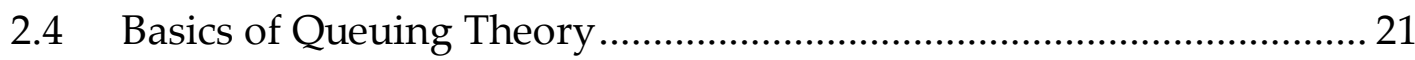

2.5 Detailed Analysis of the Akçelik Model................................................. 25

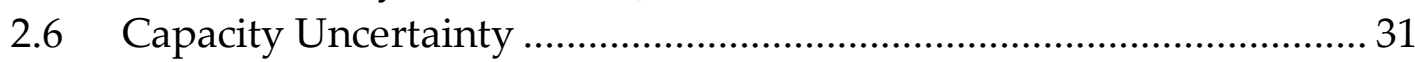

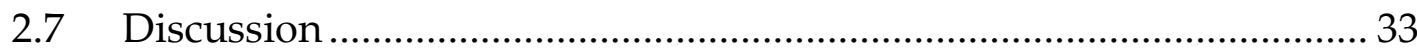

2.7.1 Gaps in Current Research............................................................. 33

2.7.2 Potential Improvements ................................................................ 33

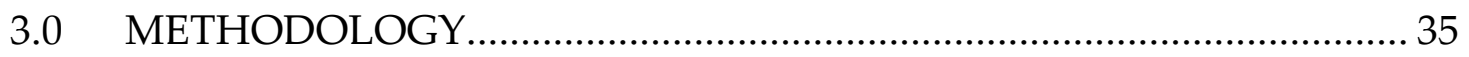

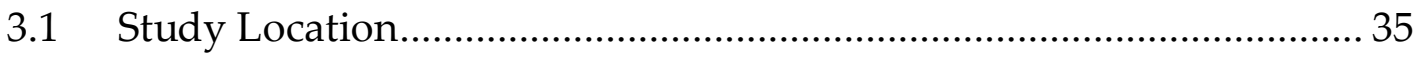

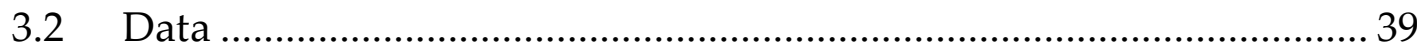

3.3 Segmentation of Congested and Uncongested Regimes ....................... 40

3.3.1 Rescaled Cumulative Curve ........................................................... 42 
vi

3.3.2 Traffic State Identification .................................................... 45

3.4 Parameter Measurement............................................................. 46

3.4.1 Travel Time Estimation......................................................... 46

3.4.2 Capacity Measurement ........................................................... 48

3.4.3 Free-Flow Speed Measurement................................................ 49

3.5 Quantile Function.................................................................... 49

4.0 DATA ANALYSIS .................................................................. 51

4.1 Measured Free-Flow Speed, Speed at Capacity, and Capacity .......... 51

4.2 Goodness of Fit Tests ................................................................... 54

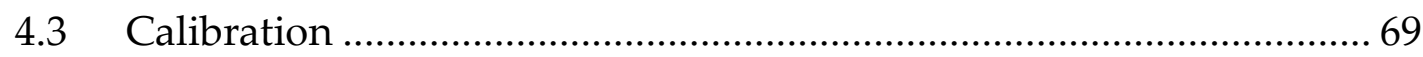

4.4 Capacity as a Random Variable ................................................... 83

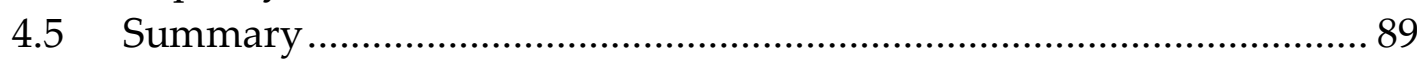

5.0 MODIFICATIONS .................................................................. 91

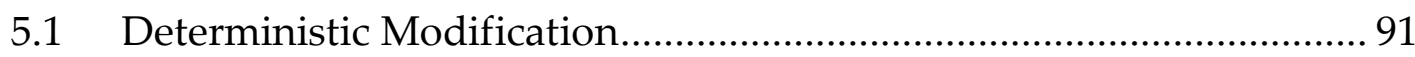

5.1.1 Speed-Flow Relationship at Link Level.................................... 96

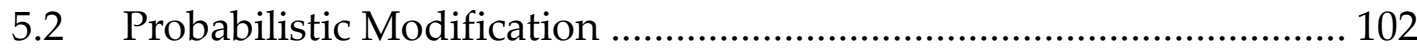

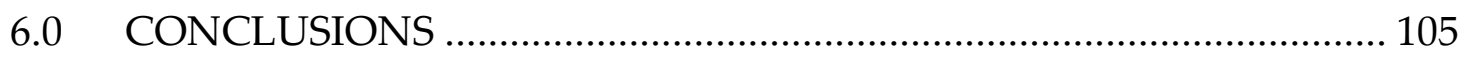

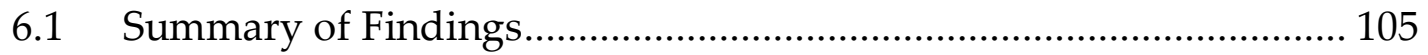

6.2 Future Work......................................................................... 106

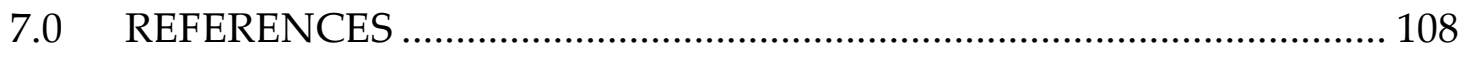

Appendices

A. DAILY CAPACITY AND FREE-FLOW SPEED ............................ 116

B. CAPACITY DISTRIBUTIONS .................................................... 120

C. TRAVEL TIME DISTRIBUTIONS ................................................. 124 
List of Tables

Table 2.1 Akçelik suggested values for $J_{A}$

Table 4.1 Goodness of fit to observed data for $\mathrm{v} / \mathrm{c}<1$

Table 4.2 Calibration results

72

Table 4.3 Comparison of the bias, RMSE, and MAE for uncalibrated and calibrated travel time estimation functions

Table 4.4 Left measured breakdown flows and maximum flows

Table 5.1 Statistical description of free-flow speed, speed at capacity, speed within the queue, breakdown flow, and maximum flow at link level

Table 5.2 Statistical description of bottleneck activation, deactivation, and duration time at link level 
List of Figures

Figure 1.1 Typical speed-flow and travel time-flow relationship $\quad 2$

Figure 2.1 Speed-flow relationships for freeway segments 10

Figure 2.2 Comparison of travel time estimation functions for v/c $<1 \quad 17$

Figure 2.3 Comparison of travel time estimation functions for v/c $>1 \quad 18$

Figure 2.4 Hypothetical arrival and departure curves 23

Figure 2.5 Stationary arrival and departure curves for oversaturated

conditions

Figure 2.6 Akçelik model versus queuing theory for $T=1 \mathrm{hr} \quad 28$

Figure 2.7 Sensitivity of the Akçelik model to T 29

Figure 2.8 Sensitivity of the Akçelik model to delay parameter 30

Figure 2.9 Sensitivity of the Akçelik model to free-flow speed 31

Figure 3.1 Portland Metropolitan Area freeway network 36

Figure 3.2 Map of ORE 217 Southbound, Portland, OR 37

Figure 3.3 Speed Map of ORE 217 Southbound, Portland, OR on

Thursday, April 8, 2010

Figure 3.4 Schematic map of the study locations: milepost 1.92

(Beaverton-Hillsdale Hwy) and milepost 3.12 (Denney Rd) 
Figure 3.5 Rescaled cumulative curve construction, ORE 217 SB,

milepost 4.35, January 22, 2010

Figure 3.6 Traffic state identification, ORE 217 SB, milepost 4.35,

January 22, 2010

Figure 3.7 Equivalent hourly flows versus average travel times plus/minus one standard deviation of travel times for left lane at milepost 4.35, on January 22th, 2010

Figure 3.8 Time-series of average flows and speeds for left lane at milepost 4.35, on January 22th, 2010

Figure $4.1 \quad$ Box plots of free-flow speed

Figure 4.2 Box plots of speed at capacity

Figure 4.3 Box plots of capacity

Figure 4.4 Comparison of travel time estimation functions against

field data for $\mathrm{v} / \mathrm{c}<1$ for the left lane at milepost 1.92

Figure 4.5 Comparison of travel time estimation functions against

field data for $\mathrm{v} / \mathrm{c}<1$ for the right lane at milepost 1.92

Figure 4.6 Comparison of travel time estimation functions against

field data for $\mathrm{v} / \mathrm{c}<1$ for the left lane at milepost 3.12 
Figure 4.7 Comparison of travel time estimation functions against

field data for $\mathrm{v} / \mathrm{c}<1$ for the right lane at milepost 3.12

Figure 4.8 Residual values for the tested equations for the left lane at milepost 1.92

Figure 4.9 Residual values for the tested equations for the right lane at milepost 1.92

Figure 4.10 Residual values for the tested equations for the left lane at milepost 3.12

Figure 4.11 Residual values for the tested equations for the right lane at milepost 3.12

Figure 4.12 Bias values for different ranges of $\mathrm{v} / \mathrm{c}$ ratio 66

Figure 4.13 RMSE values for different ranges of $\mathrm{v} / \mathrm{c}$ ratio 67

Figure 4.14 MAE values for different ranges of v/c ratio 68

Figure 4.15 Comparison of calibrated travel time estimation functions against field data for $\mathrm{v} / \mathrm{c}<1$ for the left lane at milepost 1.92 
Figure 4.16 Comparison of calibrated travel time estimation functions against field data for $\mathrm{v} / \mathrm{c}<1$ for the right lane at milepost 1.92

Figure 4.17 Comparison of calibrated travel time estimation functions against field data for $\mathrm{v} / \mathrm{c}<1$ for the left lane at milepost 3.12

Figure 4.18 Comparison of calibrated travel time estimation functions against field data for $\mathrm{v} / \mathrm{c}<1$ for the right lane at milepost 3.12

Figure 4.19 Residual values for the tested calibrated equations for the left lane at milepost 1.92

Figure 4.20 Residual values for the tested calibrated equations for the right lane at milepost 1.92

Figure 4.21 Residual values for the tested calibrated equations for the left lane at milepost 3.12

Figure 4.22 Residual values for the tested calibrated equations for the right lane at milepost 3.12 
Figure 4.23 Bias values of calibrated models for different ranges of

$\mathrm{v} / \mathrm{c}$ ratio

Figure 4.24 RMSE values of calibrated models for different ranges of $\mathrm{v} / \mathrm{c}$ ratio

Figure 4.25 MAE values of calibrated models for different ranges of

$\mathrm{v} / \mathrm{c}$ ratio

Figure 4.26 Empirical cumulative distribution of 5-minute breakdown

flows

Figure 4.27 Empirical cumulative distribution of 5-minute maximum

flows

Figure 4.28 Average on-ramp volumes at study locations

Figure 4.29 Empirical cumulative distribution of 15-minute breakdown

flows

Figure 4.30 Empirical cumulative distribution of 15-minute maximum

flows

Figure 5.1 The delay experienced by vehicle $i$ in a queue with stationary arrival and departure rates 
Figure 5.2 Empirical cumulative distributions of vehicles' speed

within the queue

Figure 5.3 Time dependent travel time and v/c ratio relationship

Figure 5.4 Empirical cumulative distributions of bottleneck activation and deactivation times over all the lanes at milepost 1.92 and milepost 3.12

Figure 5.5 Comparison of the calibrated BPR functions for $\mathrm{v} / \mathrm{c}<1$ at lane and link levels

Figure 5.6 Probabilistic volume vs. travel time relationship for the left lane at milepost 1.92 against field data from 5 days 


\subsection{INTRODUCTION}

For the past 40 years, transportation professionals have used a four-step approach in modeling transportation demand. The classical four-step urban transportation planning procedure includes trip generation, trip distribution, modal split, and traffic assignment. In this procedure, the transportation system can be viewed as a conventional economic system with demand and supply subsystems. The demand side is comprised by mode specific origindestination (O-D) matrices. The supply side of a transportation system is comprised by a network represented by links, nodes and their associated costs. Link costs are usually a function of a number of factors including link distance, free-flow speed, capacity, and a speed-flow relationship. In traffic assignment, an O-D trip matrix is loaded onto the network and a set of link flows is produced. The speed-flow relationship is important as it relates the use of the network links to link travel time and thus link travel cost (Ortúzar and Willumsen, 2001).

The relationship between speed and flow has been a topic of intense research in traffic flow theory. Assuming that traffic conditions on a given road segment are stationary and that drivers behave the same way, on 
average, under the same average conditions, a relationship between speed and flow exists $(T R B, 1975)$. A speed-flow relationship is usually presented as in figure 1.1, left. For traffic assignment applications, this relationship is presented in terms of travel time per unit distance versus flow as in figure 1.1, right.
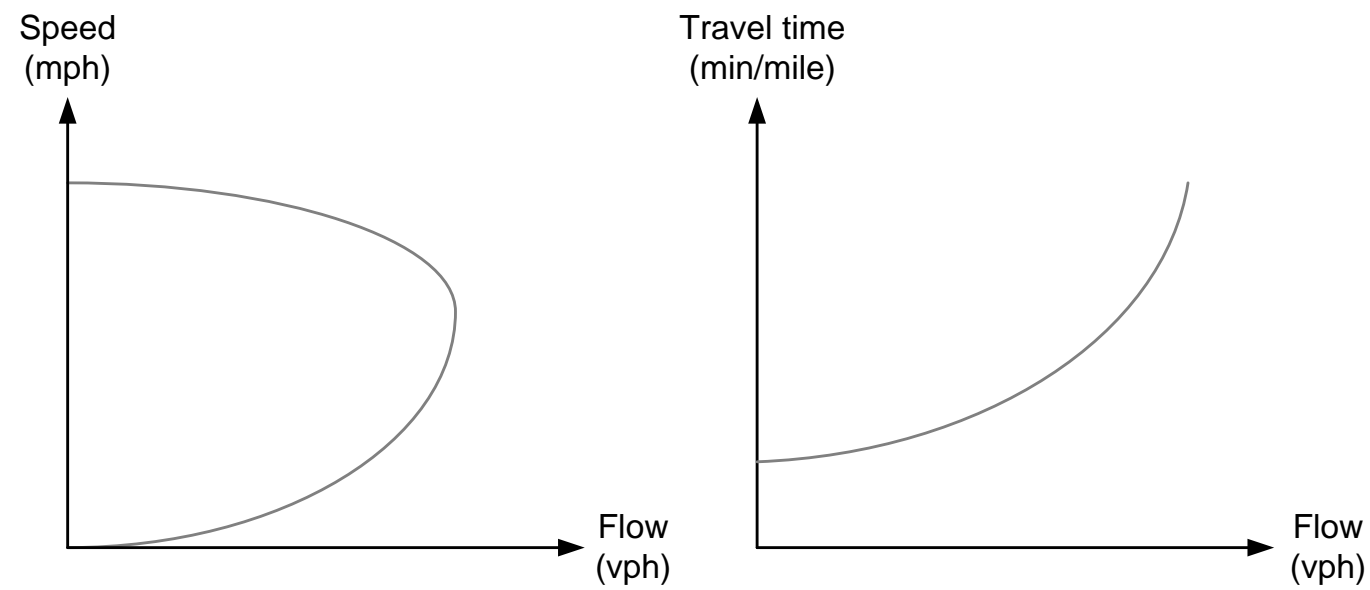

\section{Figure 1.1. Typical speed-flow and travel time-flow relationship}

It is worth mentioning that the flow used in the travel time-flow relationship for planning applications is demand flow while the flow utilized in traffic flow theory is detected flow at a section of a road. In free-flow traffic conditions, prior to queue formation, detected flow equals demand flow at the detection point. As soon as a queue forms, the usual counting procedures (by loop detectors, tubes, or etc.) are only able to count the served demand, and not the demand flow (Dowling and Skabardonis, 2008). 


\subsection{Problem Statement}

Travel demand models use speed-flow relationships to assign traffic to links in a network. Speed-flow equations currently used for planning applications do not adequately reflect travel times on transportation facilities. In addition, developments in high resolution traffic sensors over the past 50 years have provided a wealth of empirical speed-flow data. Improved speedflow relationships can be developed based on actual empirical measurements, rather than assuming constant relations over entire highway network systems. The use of volume-to-capacity (v/c) ratio based models also raises concerns about the accuracy of their predictions because existing constant capacity analysis methodologies for use in traffic assignment are inadequate. This study empirically evaluates the speed-flow relationships for $\mathrm{v} / \mathrm{c}<1$ using field data. For congested conditions $(\mathrm{v} / \mathrm{c}>1)$ a theoretical approach is taken. This research also presents an initial step toward understanding the stochastic nature of freeway capacity as well as bottleneck duration, activation, and deactivation. 


\subsection{Research Objectives}

The objective of this research is to investigate freeway speed-flow relationships and evaluate existing speed-flow equations used for planning applications. In particular, this study focuses on:

1. Testing the accuracy of different speed-flow equations using field data,

2. Calibrating existing speed-flow models, and

3. Improving existing speed-flow relationships by incorporating new concepts of queuing theory, specifically taking into account the probabilistic nature of capacity.

Note that the primary interest is not to derive an alternative speed-flow model, but to gain more insight into the relationship of travel time and flow, and particularly whether existing models estimate travel time with an acceptable accuracy. Some modifications to existing models will be also presented. 


\subsection{Thesis Overview}

\subsubsection{Main Contributions}

The two main contributions of this research are to: (1) evaluate the accuracy of existing speed-flow relationships used in planning and calibrate them for selected locations, and (2) propose modifications for speed-flow equations taking into account the variation of capacity.

\subsubsection{Organization}

This thesis is organized as follows. Chapter 2 will provide a literature review of different speed-flow relationships and discusses some of the prior work done in relation to capacity estimation. Chapter 3 will present the data used in this research as well as the methodology used to estimate travel time, capacity, and free-flow speed and to segment congested and uncongested regimes. Chapter 4 will report results of the performed data analysis, accuracy tests, calibration of speed-flow equations as well as capacity estimation. Chapter 5 will propose modifications to existing speed-flow models for congested conditions. Finally, Chapter 6 will summarize findings and discuss suggested future work. 


\subsection{LITERATURE REVIEW}

Speed-flow relationships have been studied to a large extent in the literature. The first section of this chapter provides a brief introduction about traffic assignment. The second section reviews existing speed-flow models for planning applications. The third section discusses calibration of speed-flow models. The fourth section reviews basics of queuing theory. The fifth section reviews one of the existing models, Akçelik model, in detail. In the sixth section capacity uncertainty will be discussed. To conclude this chapter, gaps in current research are discussed.

\subsection{Traffic Assignment}

Static traffic assignment (STA) has been used by planners for decades to estimate current and future use of transportation networks. A limitation of STA is its inability to capture the dynamic aspects of traffic and networks. However, due to its simplicity and relatively good results in most cases, it is still widely used. More recently, dynamic traffic assignment (DTA) models have been developed to better represent route and departure time choices in a time-varying environment. DTA loads a road network with time varying O-D demand matrices. The time-varying nature of the demand in DTA has the 
potential to better account for queuing in congested networks. However, DTA adds complexity to traffic propagation in the network. Merchant and Nemhauser (1978a, 1978b) suggested that commonly used travel time-flow equations in STA are not appropriate for dynamic networks. They suggested the use of an "exit function" to capture congestion on a link. An exit function computes the outflow as a function of the number of vehicles on the link. Exit flow functions have been criticized as they typically violate the first in-first Out (FIFO) condition on the links (Carey, 1986, 1987). Other formulations including simulation based models, cell transmission based models (Daganzo, 1994), and microscopic models have been also proposed. Temporal extension of static travel time-flow functions has been used in Janson (1991), Ran et al. (1996), and Chen and Hsueh (1998). Ben-Akiva et al. (1998) divided network links into moving and queuing sections in DynaMIT. The static function is applied only to the moving part in their simulation model. Mahmassani (2001) moves vehicles on links based on a modified Greenshield type speed-density relationship in DYNASMART. Other traffic flow models could also be applied. Since travel time-flow relationships tend to be convex, they are convenient to use in assignment procedures. 


\subsection{Speed-Flow Relationships for Planning Applications}

The most well known speed-flow equation is a function proposed by the Bureau of Public Roads (1964), known as the standard BPR function:

$$
V=\frac{V_{0}}{\left(1+a\left(\frac{v}{c}\right)^{b}\right)}
$$

where

$V=$ average travel speed $(\mathrm{mph})$,

$V_{0}=$ free-flow speed $(\mathrm{mph})$

$v=$ demand volume (vph),

$c=$ practical capacity (vph) (about 80 percent of the maximum capacity),

$a=0.15$, and $b=4$.

Note that a clear definition of capacity and ways to measure it were not

provided by the BPR. In the BPR function, parameter $a$ determines the ratio of free-flow travel time to the travel time at capacity and parameter $b$ determines how rapidly travel time increases from the free-flow travel time. Higher values of $b$ make estimated travel time less sensitive to $\mathrm{v} / \mathrm{c}$ ratio until v/c approaches 1 . For $\mathrm{v} / \mathrm{c}$ ratios greater than 1 , higher values of $b$ cause travel time to be more sensitive to v/c ratio. Dowling et al. (1997) and Dowling and 
Skabardonis (1993) empirically evaluated the standard BPR function (with $a$ $=0.15$ and $b=4)$ with field data and concluded that the standard BPR function overestimates travel times at v/c ratios between 0.8 and 1 and underestimates travel times in queued conditions. A number of limitations of the standard BPR function are identified in Skabardonis and Dowling (1997). The BPR function is based on data that do not reflect today's traffic operating conditions. It also does not take into account characteristics of different facilities. Also it highly underestimates travel times for congested conditions.

The standard BPR function was used as outlined above in the Highway Capacity Manual (HCM) (1965). The HCM (1985) described the same speedflow relationship with higher sensitivity of speeds to traffic volumes for the low volumes. The HCM (1994) described a different shape for the speed-flow relationship based on empirical observations. Most recently, the HCM (2000) describes speed-flow relationships for different classes of roads (See figure 2.1). One of the major drawbacks of the relationship proposed by the HCM (2000) is its inability to estimate speeds for v/c ratios greater than 1. A review of different speed-flow functions can be found in Branston (1976). 


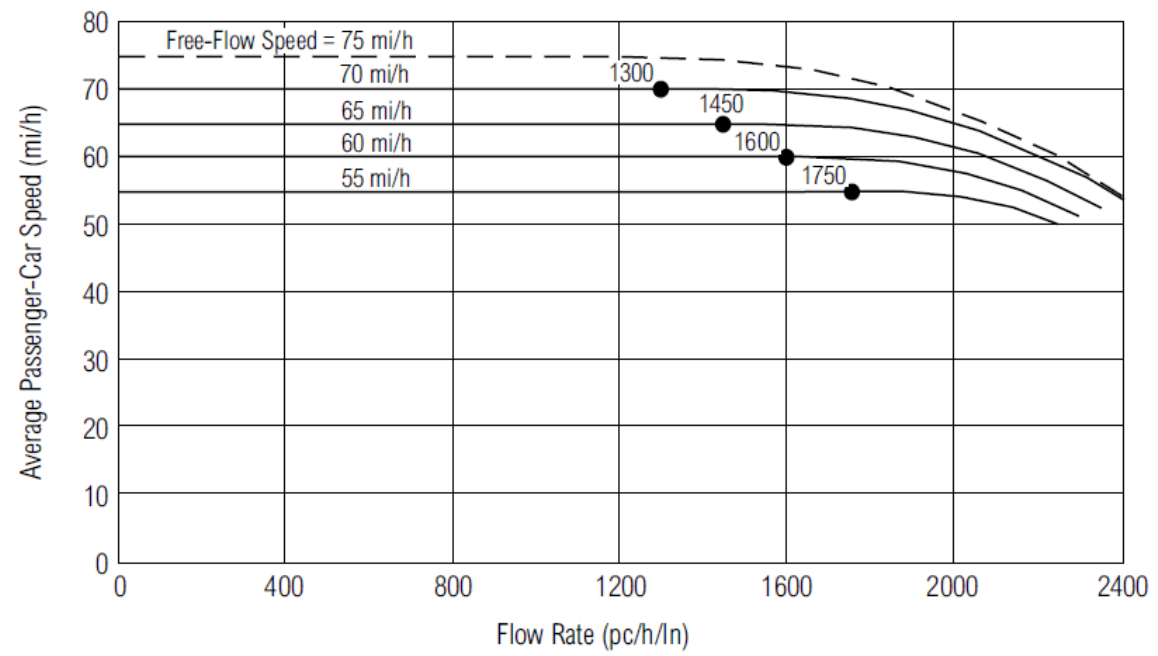

Figure 2.1. Speed-Flow Relationships for Freeway Segments (HCM, 2000)

Calibrated versions of BPR with different values of parameters $a$ and $b$ are proposed in Singh (1995), Kurth et al. (1996), Dowling et al. (1997), Skabardonis and Dowling (1997), Singh (1999), and Hansen et al. (2005). For example, the Portland metropolitan planning organization (METRO) uses a slightly modified version of the BPR function where $a=0.15$, and $b=7$ (Hansen et al., 2005):

$$
t=t_{0}\left(1+a\left(\frac{v}{0.75 c}\right)^{b}\right)
$$

where

$t=$ average travel time per unit distance, $t_{0}=$ free-flow travel time per unit distance, 
Because of a number of limitations of the BPR function, conical functions were introduced by Spiess (1990):

$$
\begin{gathered}
t=t_{0} \cdot f(x) \\
f(x)=2+\sqrt{\alpha^{2}(1-x)^{2}+\beta^{2}}-\alpha(1-x)-\beta
\end{gathered}
$$

where

$t=$ average travel time per unit distance,

$t_{0}=$ free-flow travel time per unit distance,

$x=\mathrm{v} / \mathrm{c}$ ratio (no clear definition of capacity was provided),

$\beta=\frac{2 \alpha-1}{2 \alpha-2}$, and $\alpha$ is a number greater than 1.

It is shown in Spiess (1990) that the conical function compared to the standard BPR function has better computational efficiency in the assignment process, in spite of its apparently more complex formula. METRO uses a calibrated version of the conical function where $\alpha=7$ and $\beta=1.0833$ (Hansen et al., 2005).

Davidson (1966, 1978) proposed a travel time function based on concepts of queuing theory:

$$
t=t_{0}\left[1+\frac{J_{D} X}{(1-X)}\right]
$$


where

$t=$ average travel time per unit distance,

$t_{0}=$ free-flow travel time per unit distance,

$X=$ degree of saturation (volume-to-capacity ratio; no clear definition of capacity was provided), and

$J_{D}=$ delay parameter (time per unit distance).

Davidson derived equation 2.5 from the steady-state delay formulation for a single channel queuing system with random arrival rates and exponentially distributed service rates. In this system, delay can be calculated as follows:

$$
d=\frac{1}{Q}+\frac{X}{Q(1-X)}
$$

where

$d=$ delay (time unit),

$Q=$ capacity $(\mathrm{veh} / \mathrm{hr})$, and

$X=$ degree of saturation (volume-to-capacity ratio).

The first term in equation 2.6 represents the service time and the second term represents the queuing delay. To obtain equation 2.5 from equation 2.6, 
Davidson multiplied the queuing delay term by a delay factor $\left(J_{D}\right)$ and assumed that the service time equals free-flow travel time.

$$
t_{0}=\frac{1}{Q}
$$

The Davidson equation has a definitional inconsistency which was first raised by Golding (1977) and then further discussed by Akçelik (1991). The inconsistency of the Davidson equation is due to its implication that the capacity can be defined as the inverse of free-flow travel time and the degree of saturation as volume times free-flow travel time, which is meaningless. Davidson function estimates finite travel times for v/c smaller than 1 and negative travel times for $\mathrm{v} / \mathrm{c}$ greater than 1 . It estimates an infinite travel time for $\mathrm{v} / \mathrm{c}$ equal to 1 .

Akçelik (1991) developed a time-dependent version of Davidson's function using the steady-state delay equation for a single channel queuing system:

$$
t=t_{0}+0.25 T\left[(X-1)+\sqrt{(X-1)^{2}+\frac{8 J_{A} X}{c T}}\right]
$$

where

$t=$ average travel time per unit distance, 
$t_{0}=$ free-flow travel time per unit distance,

$X=$ degree of saturation (volume-to-capacity ratio; no clear definition of capacity was provided),

$T=$ duration of analysis period $(\mathrm{h})$,

$c=$ capacity $(\mathrm{vph})$, and

$J_{A}=$ delay parameter (unitless).

The delay parameter $J_{A}$ corresponds to the quality of service provided by the road section and is independent of the traffic flow but sensitive to the value of travel time at capacity (Akçelik, 1994; Dowling et al., 2004). A detailed analysis of the Akçelik equation is presented in section 2.4.

Chapter 30 of the HCM 2000 suggests the following modified speedflow equation:

$$
t=t_{0}+D_{q}+0.25 T\left[(X-1)+\sqrt{(X-1)^{2}+\frac{16 J L^{2} X}{T^{2}}}\right]
$$

where

$t=$ average travel time $(\mathrm{h})$,

$t_{0}=$ free-flow travel time $(\mathrm{h})$,

$D_{q}=$ delay due to leftover queue from prior hour (h),

$X=$ degree of saturation (volume-to-capacity ratio), 
$T=$ duration of analysis period $(\mathrm{h})$,

$L=$ segment length (mi), and

$J$ = delay parameter $\left(h^{2} / m i^{2}\right)$.

The delay caused by leftover queue is computed as follows as in Dowling et al. (2004):

$$
D_{q}=\frac{Q(1+u) t}{2 c T}
$$

where

$Q=$ leftover queue from prior period (veh),

$c=$ capacity $(\mathrm{veh} / \mathrm{hr})$,

$t=$ duration of unserved demand; $t=0$ if $Q=0$ else $t=\min \left\{T, \frac{Q}{c[1-\min (1, X)]}\right\}$

$u=$ delay parameter; $u=0$ if $\mathrm{Q}=0$ else $u=1-\frac{c T}{Q}[1-\min (1, X)]$

Dowling and Skabardonis (2008) proposed a simplified version of the Akçelik equation as follows:

$$
t=t_{0}+0.25 T\left((X-1)+\sqrt{(X-1)^{2}+J X}\right)
$$

where all variables are the same as defined before in equation 2.9. Parameter $J$ in equation 2.11 is unitless. 
In this equation, the constant multiplier 8 for the Akçelik $J_{A}$ parameter is subsumed within the $J$ calibration parameter itself and the variable capacity is dropped. An hour-long analysis period is assumed.

A study by Dowling and Skabardonis (2008) showed that the Akçelik curve is consistent with the theoretical delay due to queuing for $\mathrm{v} / \mathrm{c}>1$. $T$ in the Akçelik equation is the time interval during which a constant flow rate persists. In addition, the Akçelik equation assumes no initial queue at the start of the flow period. However, the extra delay caused by the leftover queue from the prior period can be calculated and added to the Akçelik equation as in Dowling et al. (2004) and HCM (2000).

Figure 2.2 shows a comparison of the standard BPR, METRO updated BPR, METRO conical, Davidson, Akçelik, and the HCM 2000 speed-flow curves with a free-flow speed of $60 \mathrm{mph}$, link length of 1 mile, 1 hour long analysis period, and a capacity of 2,300 vehicle per hour per lane (vphpl) for v/c smaller than 1. Delay parameters $J_{A}=0.1$ and $J=0.04 h r^{2} / m i^{2}$ are used for the Akçelik and HCM 2000 equations, respectively. The Akçelik curve closely matches the HCM 2000 curve. The Davidson and METRO conical models predicts higher average travel times when compared to the other models. The travel times predicted by the standard BPR and METRO BPR 
models are constantly higher than those predicted by Akçelik and HCM2000 except for v/c ratios close to 1.

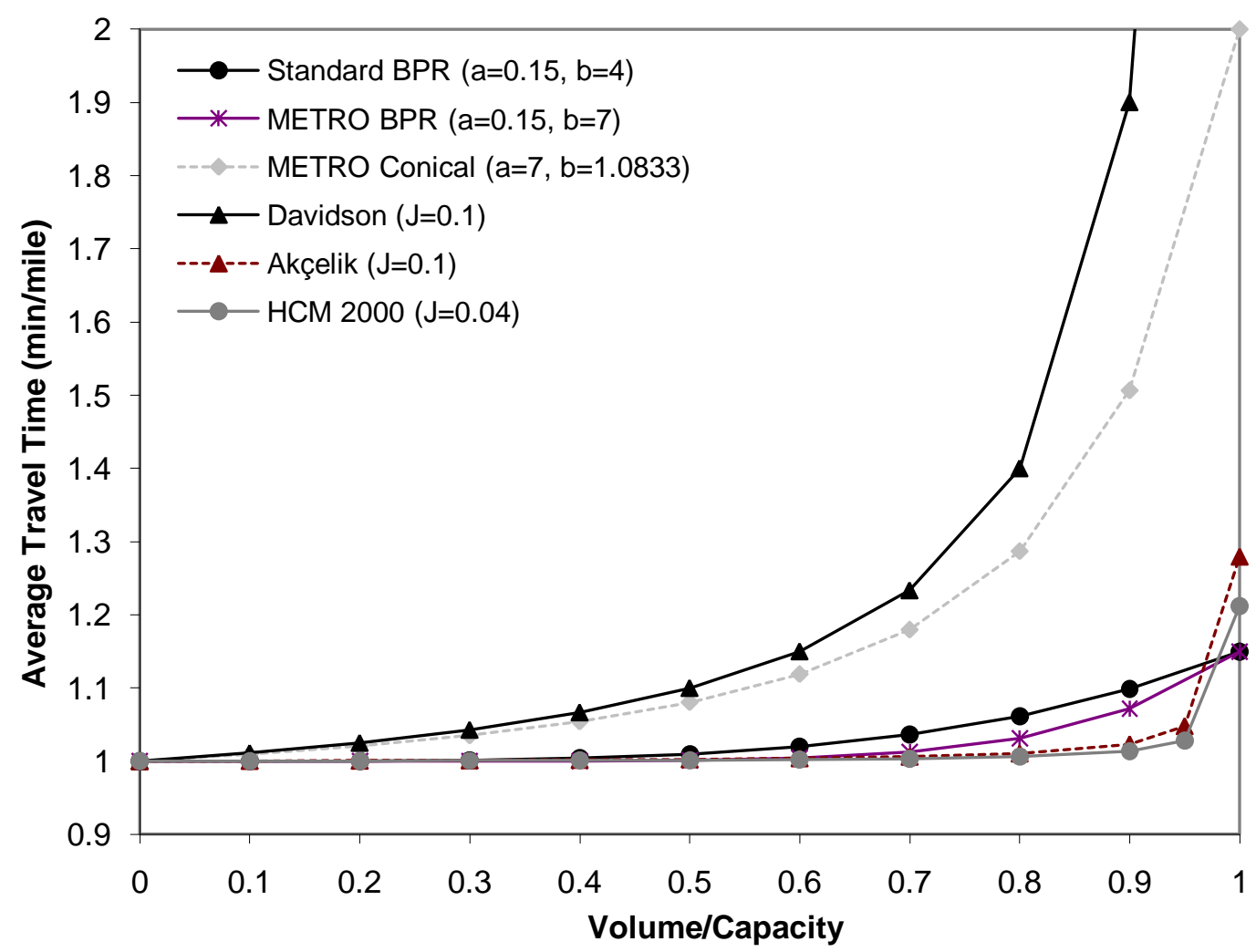

Figure 2.2. Comparison of travel time estimation functions for v/c $<1$.

Figure 2.3 compares the same travel time estimation functions for v/c greater than 1. The Davidson function is excluded from this figure since it only estimates travel times for v/c smaller than 1. The Akçelik curve closely matches the HCM2000 curve. Travel times estimated by the Akçelik and HCM2000 models increase almost linearly for v/c ratios greater than 1 . While the BPR curves increase nonlinearly. For METRO BPR formulation (with 
$a=0.15$ and $b=7$ ), the travel time estimates increase rapidly with higher $v / c$ ratios. The conical function behaves as a quasi-linear function for large v/c ratios.

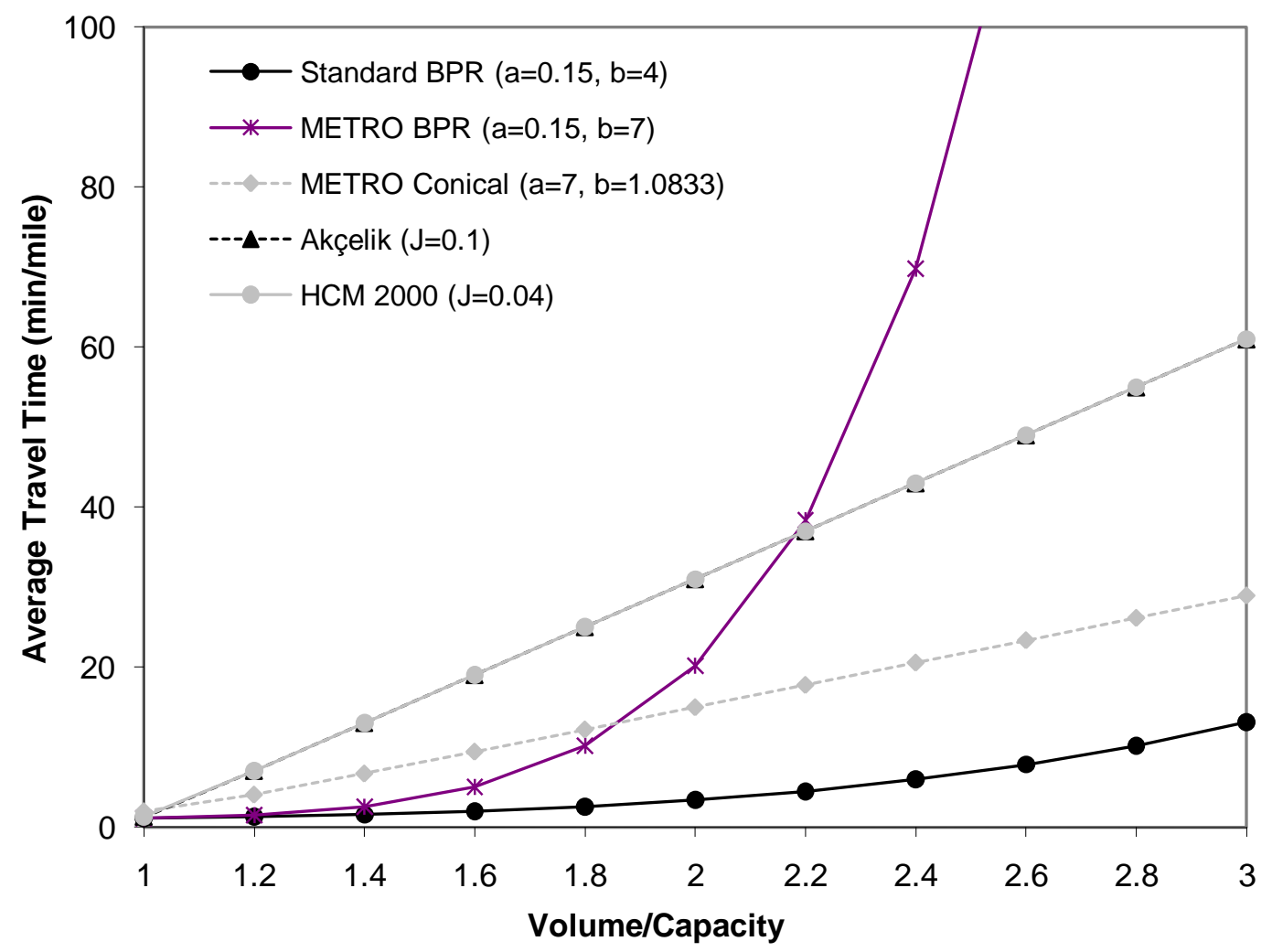

Figure 2.3. Comparison of travel time estimation functions for $v / c>1$.

\subsection{Calibration of Speed-Flow Models}

In the literature, calibration of speed-flow equations has not been given enough attention despite its importance. As mentioned earlier, calibrated versions of BPR, fitted to speed-flow data from different sites, with different 
values of parameters $a$ and $b$ are proposed in Singh (1995), Kurth et al. (1996), Dowling et al. (1997), Skabardonis and Dowling (1997), Singh (1999), and Hansen et al. (2005). As mentioned earlier, currently METRO uses an updated version of the BPR function where $a=0.15$, and $b=7$ (Hansen et al., 2005).

The conical function like all the other models presented can be calibrated by fitting its curve to observed speed-flow data. METRO has developed a calibrated version of the conical function where $\alpha=7$ and $\beta=1.0833$ (Hansen et al., 2005).

The delay parameter $\left(J_{A}\right)$ in the Akçelik model corresponds to the quality of service on the road which depends on the frequency of delaycausing elements in the road section. To obtain rough estimates of the delay parameter, Akçelik provided the following formula:

$$
J_{A}=\frac{2 c}{T}\left(t_{C}-t_{0}\right)^{2}
$$

where $t_{C}$ is the value of travel time at capacity (in hours per mile) and the rest of the variables are the same as defined before in equation 2.8. For example, using $\frac{t_{C}}{t_{0}}=1.5, c=2,000 \mathrm{vphpl}, T=1$ hour, and a free-flow speed of $60 \mathrm{mph}$, 
$J_{A}=0.28$ is found. If $\frac{t_{C}}{t_{0}}=2$, then for the same values of capacity and free-

flow speed, $\quad J_{A}=1.11$. This indicates the sensitivity of the delay parameter to the travel time at capacity. Another appropriate way to calibrate the Akçelik function to determine the value of the delay parameter is using the data points for medium and high flow conditions $(0.4<\mathrm{v} / \mathrm{c}<0.9)$ but not oversaturated conditions (Akçelik, 1991). Table 2.1 shows the suggested values by Akçelik for $J_{A}$.

Table 2.1. Akçelik suggested values for $J_{A}($ Akçelik, 1991)

\begin{tabular}{|c|c|c|c|c|c|}
\hline \multirow[t]{2}{*}{ Road type } & \multicolumn{2}{|c|}{$\begin{array}{l}\text { Free-Flow } \\
\text { Speed }\end{array}$} & \multirow{2}{*}{$\begin{array}{l}\text { Capacity } \\
\text { (vphpl) }\end{array}$} & \multirow{2}{*}{$\frac{t_{C}}{t_{0}}$} & \multirow{2}{*}{$J_{A}$} \\
\hline & $(\mathrm{km} / \mathrm{h})$ & (mph) & & & \\
\hline Freeway & 120 & 74.6 & 2,000 & 1.59 & 0.1 \\
\hline $\begin{array}{l}\text { Arterial } \\
\text { (uninterrupted) }\end{array}$ & 100 & 62.1 & 1,800 & 1.75 & 0.2 \\
\hline $\begin{array}{l}\text { Arterial } \\
\text { (interrupted) }\end{array}$ & 80 & 49.7 & 1,200 & 2.04 & 0.4 \\
\hline $\begin{array}{l}\text { Secondary } \\
\text { (interrupted) }\end{array}$ & 60 & 37.3 & 900 & 2.27 & 0.8 \\
\hline $\begin{array}{l}\text { Secondary (high } \\
\text { friction) }\end{array}$ & 40 & 24.9 & 600 & 2.44 & 1.6 \\
\hline
\end{tabular}

The delay parameter $(J)$ in the HCM 2000 model relates to the travel time of traffic when demand is equal to capacity and free-flow travel time. Substituting $\quad X=1$ in equation 2.9 and solving for $J$ yields equation 2.13: 


$$
J=\frac{\left(t_{C}-t_{0}-D_{q}\right)^{2}}{L^{2}}
$$

where all variables are the same as defined before in equation 2.9.

A number of studies have addressed some of the calibration issues as well as variation in capacity, demand, and travel time (Hurdle et al., 1997; Tarko and Tian, 2003; Olstam et al., 2008). A study by Taylor (1984) suggested including dynamic components in speed-flow equations. Waller et al. (2001) addressed the impact of demand uncertainty on the evaluation of network improvements using stochastic traffic assignment. A recent study by $\mathrm{Tu}$ et al. (2007) empirically investigated travel time variability as a function of freeway inflow. Studies exploring the stochastic nature of freeway capacity will be discussed in section 2.5 .

\subsection{Basics of Queuing Theory}

When arrival rate exceeds departure rate at a specific location and for a period of time, a queue is formed. Traditionally, queuing analysis requires five basic elements (May, 1990):

1. Arrival rate

2. Arrival distribution

3. Service rate 
4. Service distribution

5. Queue discipline

Arrival and service rates are usually expressed as flow rates. Arrival and service rates can be specified as constants or random variables. Queuing discipline represents the order to get service. The most commonly used queue discipline in traffic operations is "first in, first out".

Generally, queuing analysis is classified into two categories: deterministic queuing analysis and stochastic queuing analysis. If arrival rates and departure rates are constant then deterministic queuing analysis is selected. If arrival rates or departure rates are random variables then stochastic queuing analysis is selected (May, 1990).

In traffic analysis, cumulative plots are commonly used for graphical representation. A cumulative plot "is the graph of a function $N(t)$ that gives the cumulative number of vehicles (or other moving objects) to have passed an observer by time $t$ starting from an arbitrary initial count, e.g. at $t=0^{\prime \prime}$ (Daganzo, 2007). Figure 2.4 shows hypothetical arrival and departure curves observed at two locations absolutely close to each other where $A(t)$ and $D(t)$ are arrival and departure curves observed upstream and downstream of a bottleneck. $Q(t)$ is defined as the number of vehicles in between the two 
locations at time $t$ (See equation 2.14) and $w(N)$ represent a trip time through the system for the Nth vehicle (See equation 2.15).

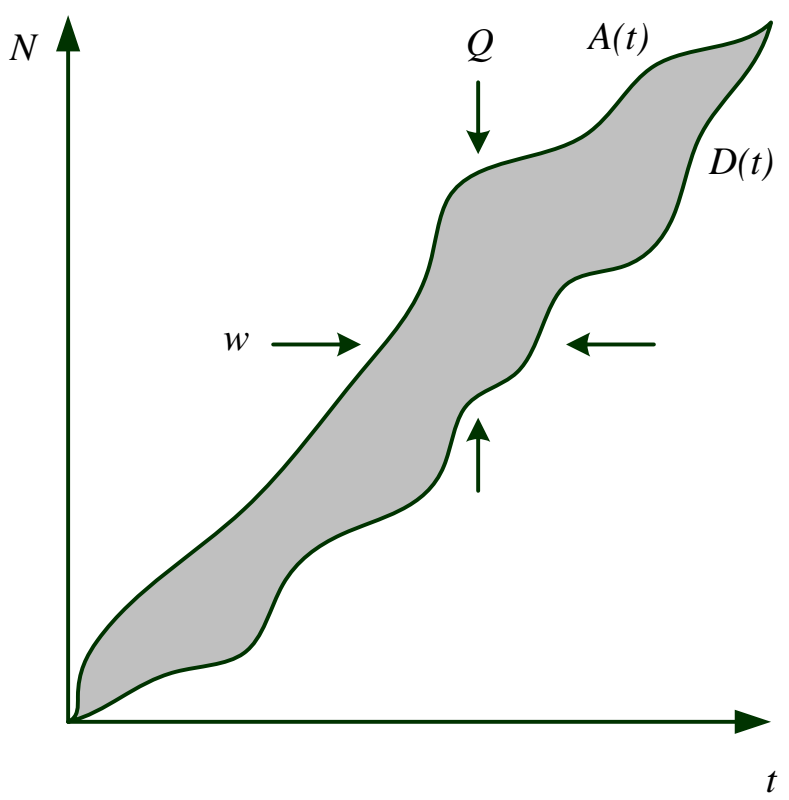

Figure 2.4. Hypothetical arrival and departure curves.

$$
\begin{gathered}
Q(t)=A(t)-D(t) \\
w(N)=D^{-1}(N)-A^{-1}(N)
\end{gathered}
$$

where $D^{-1}(N)$ is the inverse of $D(t)$ and $A^{-1}(N)$ is the inverse of $A(t)$.

The shaded area in figure 2.4 is the total wait time in the system:

$$
\text { Area }=\int_{t} Q(t) d t=\int_{t}(A(t)-D(t)) d t
$$

It should be noted that the queuing analysis presented graphically in figure 2.4 only allows to predict the queuing process in a system in a very 
short term where the two observers are located close to each other. This analysis method assumes vehicles are stacked on top of each other and form a "point" queue next to the bottleneck. However, in the real world, a queue takes considerable physical space and backs up. Therefore, a different analysis method is required to consider the actual characteristics of a queue.

A method using a "virtual arrival curve" is described in Daganzo (2007). This method assumes that the queue forms directly upstream of the bottleneck and vehicles travel at an average speed $v_{q}$ within the queue, $d_{q}$ is defined as the length of the physical queue (in distance units) and $t_{q}$ is defined as the queuing time of any vehicle. It is also assumed that vehicles approach the queue at a free-flow speed $v_{f}$. For the $d_{q}$ and $t_{q}$ of any vehicle, the following relationships exist:

$$
\begin{gathered}
t_{q}=\frac{d_{q}}{v_{q}} \\
w=t_{q}-\frac{d_{q}}{v_{f}}
\end{gathered}
$$

Equation 2.18 expresses that the delay of any vehicle is the difference between its travel time within the queue and the time that it would take to 
travel the same distance at free-flow speed. Therefore, by substituting $\frac{d_{q}}{v_{q}}$ with $t_{q}$, the queuing time $\left(t_{q}\right)$ is calculated as follows:

$$
t_{q}=\frac{w}{\left(1-\frac{v_{q}}{v_{f}}\right)}
$$

Equation 2.19 can be simplified:

$$
\begin{gathered}
t_{q}=\phi \times w \\
\phi=\frac{1}{\left(1-\frac{v_{q}}{v_{f}}\right)} \geq 1
\end{gathered}
$$

Since parameter $\phi$ is only related to the free-flow speed and the average speed of vehicles within the queue, it is the same for all the vehicles within the queue. However it is a random variable. For details see Daganzo (2007).

\subsection{Detailed Analysis of the Akçelik Model}

Akçelik model is a modified version of Davidson's function with a different definition of the delay parameter and applicable even when $\mathrm{v} / \mathrm{c} \geq 1$. The Akçelik equation is developed from the steady-state delay equation for a single channel queuing system in a time-dependent format using a coordinate 
transformation method (Akçelik, 1991). It assumes a constant arrival rate and no initial queue at the start of the analysis period. The Akçelik curve increases near linearly when demand exceeds capacity. The slope of the Akçelik curve for situations where $\mathrm{v} / \mathrm{c} \geq 1$ depends on the chosen value of $T$ (time period of analysis in hours). For example, for analysis periods of one hour in length, the slope of the Akçelik curve with respect to $X$ (v/c ratio) approaches (but never exactly matches) the slope of $1 / 2$ for large values of $X$.

A simple queuing analysis is done to explore the consistency of the Akçelik model with the classical queuing theory (See figure 2.5). Assuming an analysis period length of $T$, a constant arrival rate of $V$, and a constant departure rate of $\mathrm{Q}$, the total delay $\left(D_{T}\right)$ is calculated as follows:

$$
D_{T}=\frac{1}{2} V T^{2}\left(\frac{V}{Q}-1\right)
$$




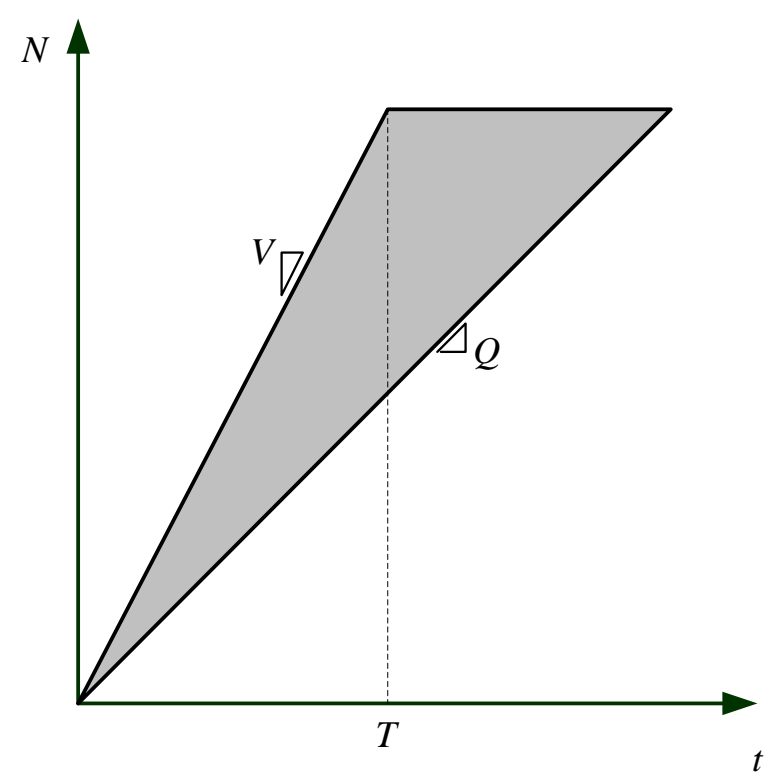

Figure 2.5. Stationary arrival and departure curves for oversaturated conditions.

Replacing $\frac{V}{Q}$ by $\mathrm{X}$, equation 2.22 can be rewritten as:

$$
D_{T}=\frac{1}{2} V T^{2}(X-1)
$$

The average delay $\left(D_{A}\right)$ can be calculated as:

$$
D_{A}=\frac{1}{2} T(X-1)
$$

Equation 2.25 shows the derivative of the average delay with respect to X. For an analysis period of $\mathrm{T}=1$ (in time unit), the slope of the average delay equals $\frac{1}{2}$. 


$$
\frac{d D_{A}(T X)}{d X}=\frac{1}{2} T
$$

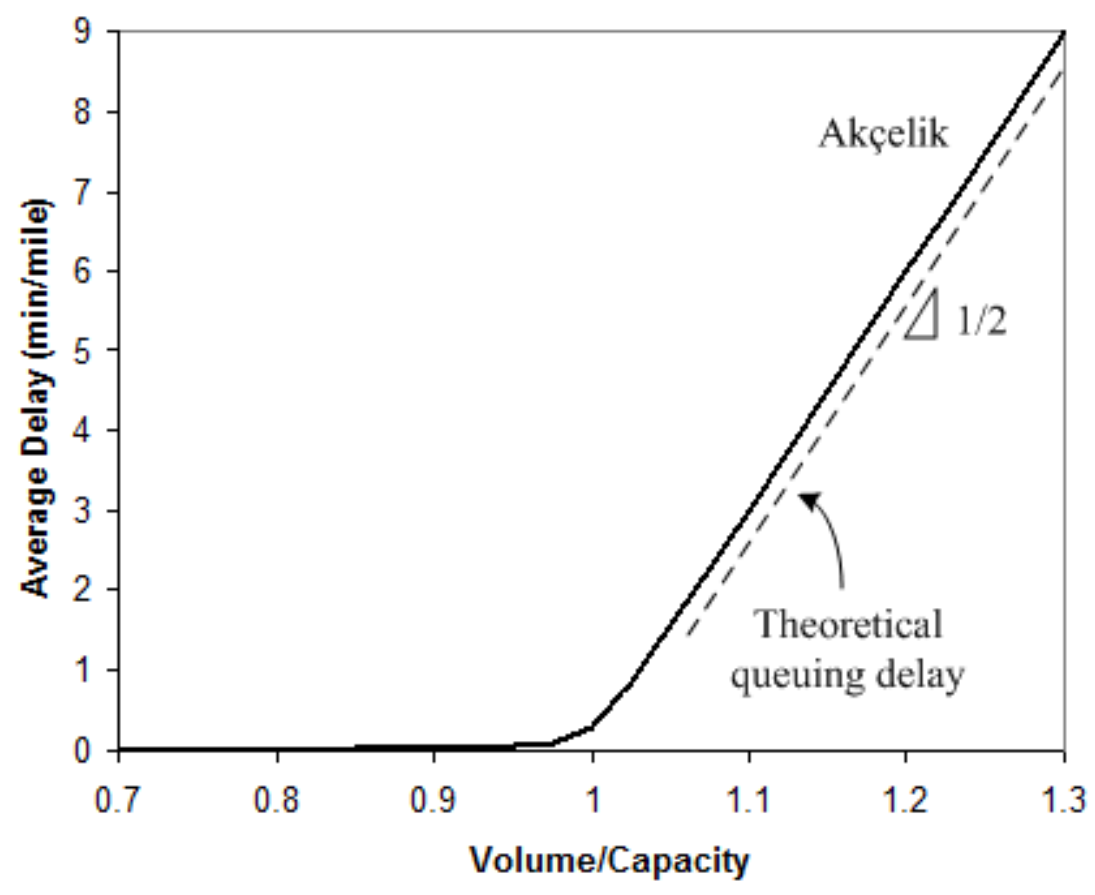

Figure 2.6. Akçelik model versus queuing theory for $T=1 \mathrm{hr}$.

Figure 2.6 illustrates the average delay estimated by Akçelik model versus the average delay according to the deterministic queuing theory. As can be seen, for v/c greater than 1, the Akçelik curve is consistent with the deterministic queuing delay. A similar analysis can be found in Dowling and Skabardonis (2008). 


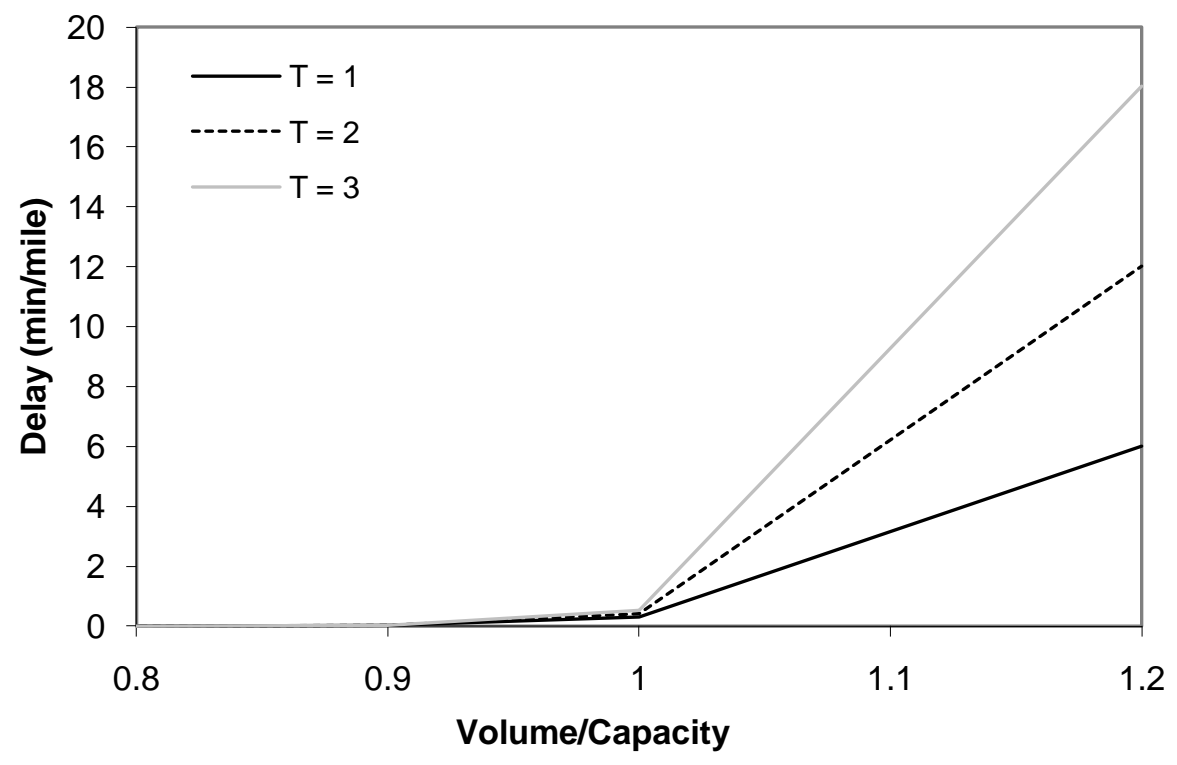

Figure 2.7. Sensitivity of the Akçelik model to $T$.

Figure 2.7 shows the sensitivity of the Akçelik model to analysis time period of T. Analysis time period is defined in Akçelik (1991) as "the time period which an average demand flow rate persists." As mathematically shown earlier in equation 2.25, increasing the value of $T$ increases the sensitivity of Akçelik model to v/c ratio. Figure 2.6 illustrates this graphically. 


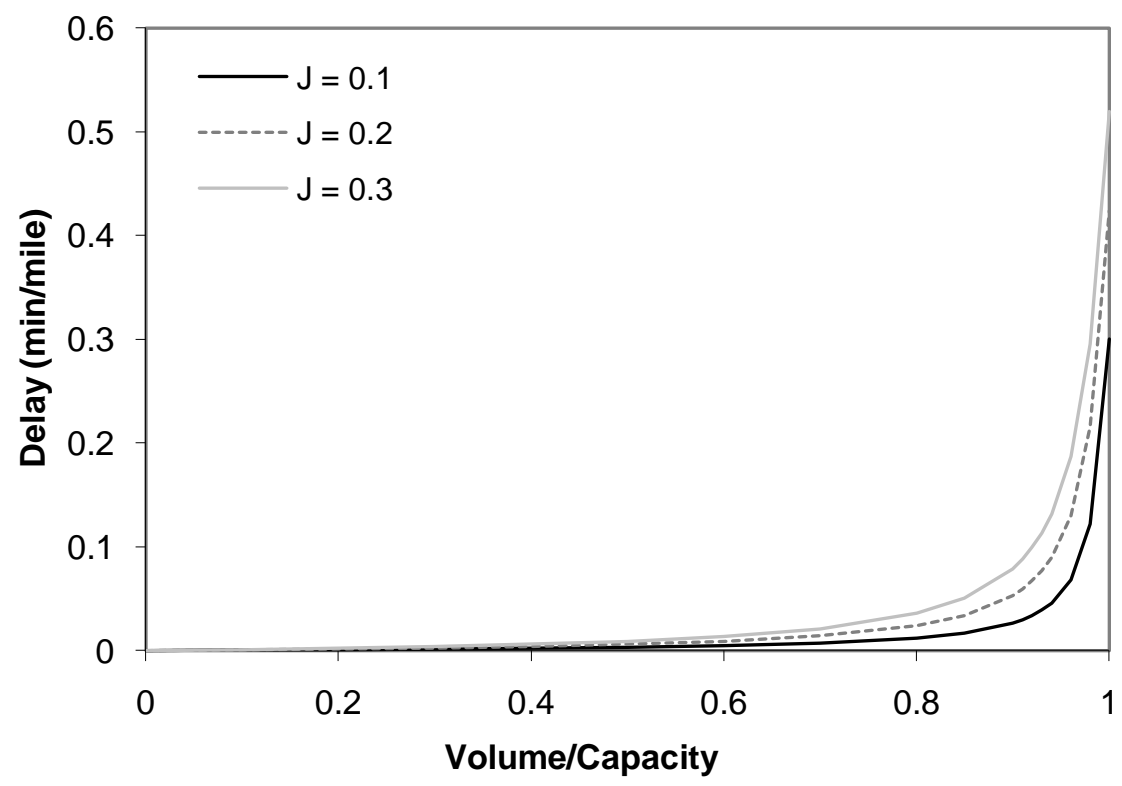

Figure 2.8. Sensitivity of the Akçelik model to delay parameter.

Figure 2.8 shows the sensitivity of the Akçelik model to delay parameter $J_{A}$. The delay parameter in the Akçelik model corresponds to the quality of service on the road which depends on the frequency of delaycausing elements (such as on-ramps, off-ramps, weaving sections, etc) in the road section. Increasing the value of $J_{A}$ increases the sensitivity of the Akçelik model to the v/c ratio. It also increases the value of travel time at capacity.

Figure 2.9 shows the sensitivity of the Akçelik model to free-flow speed (FFS). As can be seen, poor choice of free-flow speed greatly affects travel time estimates by the Akçelik model when $\mathrm{v} / \mathrm{c}<1$. 


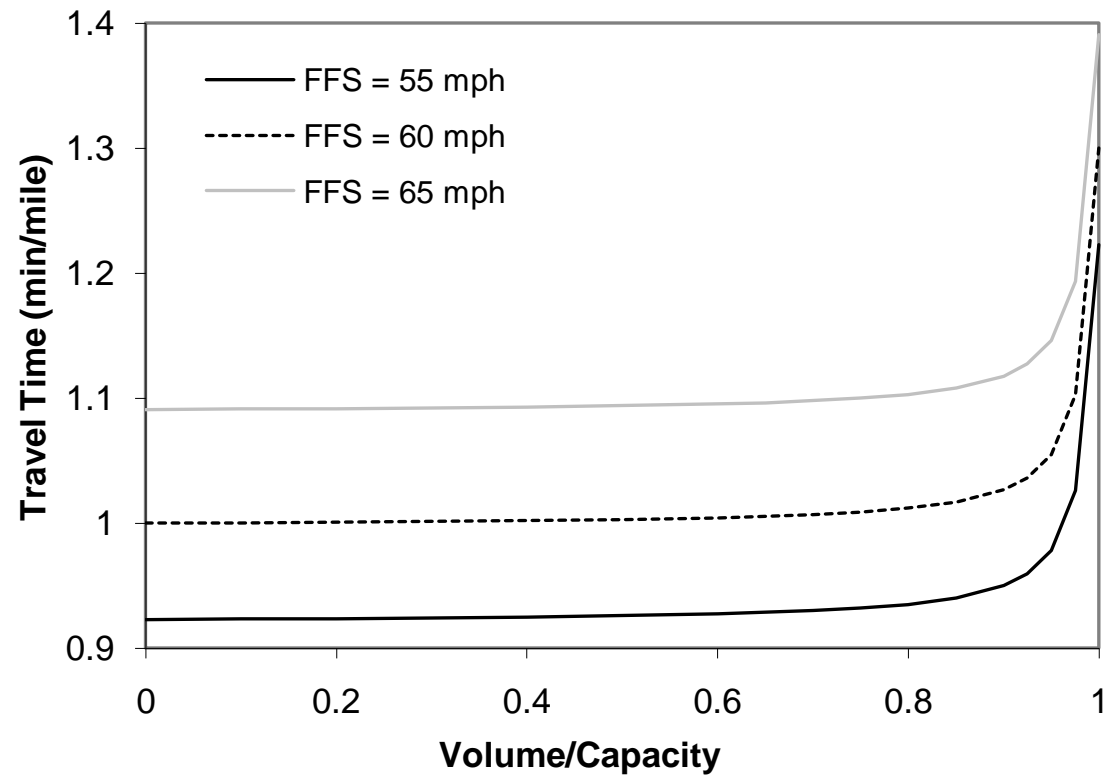

Figure 2.9. Sensitivity of the Akçelik model to free-flow speed.

\subsection{Capacity Uncertainty}

The proper definition and quantification of capacity have been topics of debate among researchers for debates. For example, capacity has been defined as "a description of the limit of the vehicle-carrying ability of a roadway" (Lorenz and Elefteriadou, 2001). HCM (2000) defines freeway capacity as "the maximum sustained 15-min rate of flow, expressed in passenger car per hour per lane (pcphpl), that can be accommodated by a uniform freeway segment under prevailing traffic and roadway conditions in a specified direction." Freeway capacity is also defined as a bottleneck's long-run queue discharge 
rate (Agyemang-Duah and Hall, 1991; Banks, 1990, 1991; Cassidy and Bertini, 1999). Several research efforts have studied the relationship between flow breakdown and capacity (Elefteriadou et al., 1995; Persaud et al., 1998; O'Leath, 1998).

Traffic breakdown "describes freeway operation near a bottleneck entrance during a period when there is a change from operation with vehicles flowing freely to operation with a queue present" (Persaud et al., 1998). Empirical studies have shown that flow breakdown does not necessarily occur at the nominal maximum flow (Elefteriadou et al., 1995; Persaud et al., 1998; Kerner, 2000; Evans et al., 2001). Breakdown flow may be lower or higher than the maximum flow.

Traditionally, the capacity of a freeway facility is treated as a constant value. However, several studies show that freeway capacity has a stochastic nature (Minderhoud, 1997; Persaud, 1998; Lorenz and Elefteriadou, 2001; Brilon et al., 2007; Geistefelft, 2008; Dong and Mahmassani, 2009). Taking into account the probabilistic nature of capacity and flow breakdown, Lorenz and Elefteriadou (2001) suggested a new definition of capacity as the rate of flow (expressed in pcphpl) along a uniform freeway segment corresponding to the expected 
probability of breakdown deemed acceptable under prevailing traffic and road conditions in a specified direction.

\subsection{Discussion}

\subsubsection{Gaps in Current Research}

In the literature, there are few studies examining the goodness of fit of the existing speed-flow models utilize empirical data. Also, calibration of speed-flow equations has been given less attention despite its importance. Although several studies have explored the stochastic characteristics of capacity (Minderhoud, 1997; Persaud, 1998; Lorenz and Elefteriadou, 2001; Brilon et al., 2007; Geistefelft, 2008; Dong and Mahmassani, 2009) and probabilistic queuing models have been developed (Viti and van Zuylen, 2010) there has been no improvement in existing travel time estimation models that take into account the variation in capacity.

\subsubsection{Potential Improvements}

The main question of this research is: "How can traffic data be used to improve travel time estimation models?" Using data from the Portland Oregon Regional Transportation Archive Listing (PORTAL), the accuracy of 
existing speed-flow models can be examined and more site-specific models can be developed. Building on previous studies, the stochastic nature of capacity can also be explored. Toward this end, existing speed-flow models can be calibrated and modified to estimate more accurate travel times and to consider variation in variables such as capacity. Queuing theory can be applied to modify existing models to take into account the time-varying flows used in dynamic traffic assignment. 


\subsection{METHODOLOGY}

This chapter presents the data, study location, and methods used in this research. The first section describes the study location. In the second section, data used in this research are described. In the third section, the method used for the segmentation of congested and uncongested regimes will be described. The fourth section discusses the methods used to measure travel time, capacity, and free-flow speed. In the fifth section, to capture the stochastic nature of capacity, the quantile function is introduced.

\subsection{Study Location}

The freeway system in the Portland metropolitan region consists of several Interstate and U.S. highways and state routes. OR-217 is a 7-mi corridor that serves the western suburbs of Portland. It is a limited access freeway that connects U.S. Route 26 and Interstate 5 (See Figure 3.1). Two study locations are used here. Both are segments of OR-217 (southbound). 


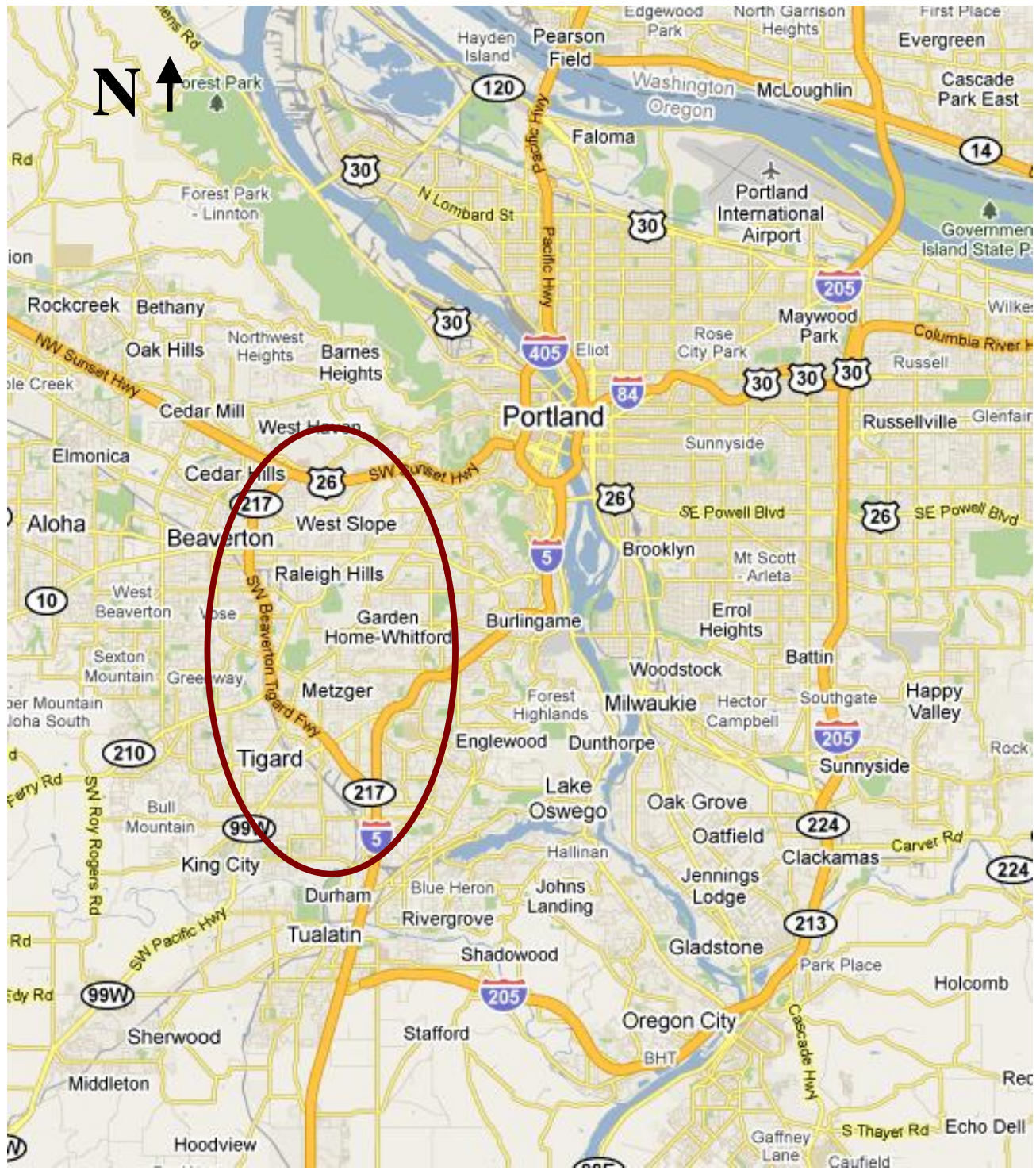

Figure 3.1. Portland Metropolitan Area freeway network

Typically, queues form on the OR-217 southbound during morning and afternoon peak periods. In the morning peak period, a recurrent bottleneck (a point where traffic flow is restricted due to the merging/separation of freeway traffic) is located between Scholls-Ferry Road and Greenburg Road, and the 
resulting queue propagates over $4-5 \mathrm{mi}$ upstream. The bottleneck activates as a result of a large inflow from the onramp at Scholls-Ferry Road and remains active from 7 a.m. to 9 a.m. During the afternoon peak, a queue forms between Denney Road and Allen Boulevard and propagates several miles upstream (often to Barnes Road). However, a queue from this active bottleneck is often overridden by another queue that forms on I-5 southbound and spills over to OR-217 southbound (Ahn et al., 2007) (See figure 3.2 and figure 3.3).
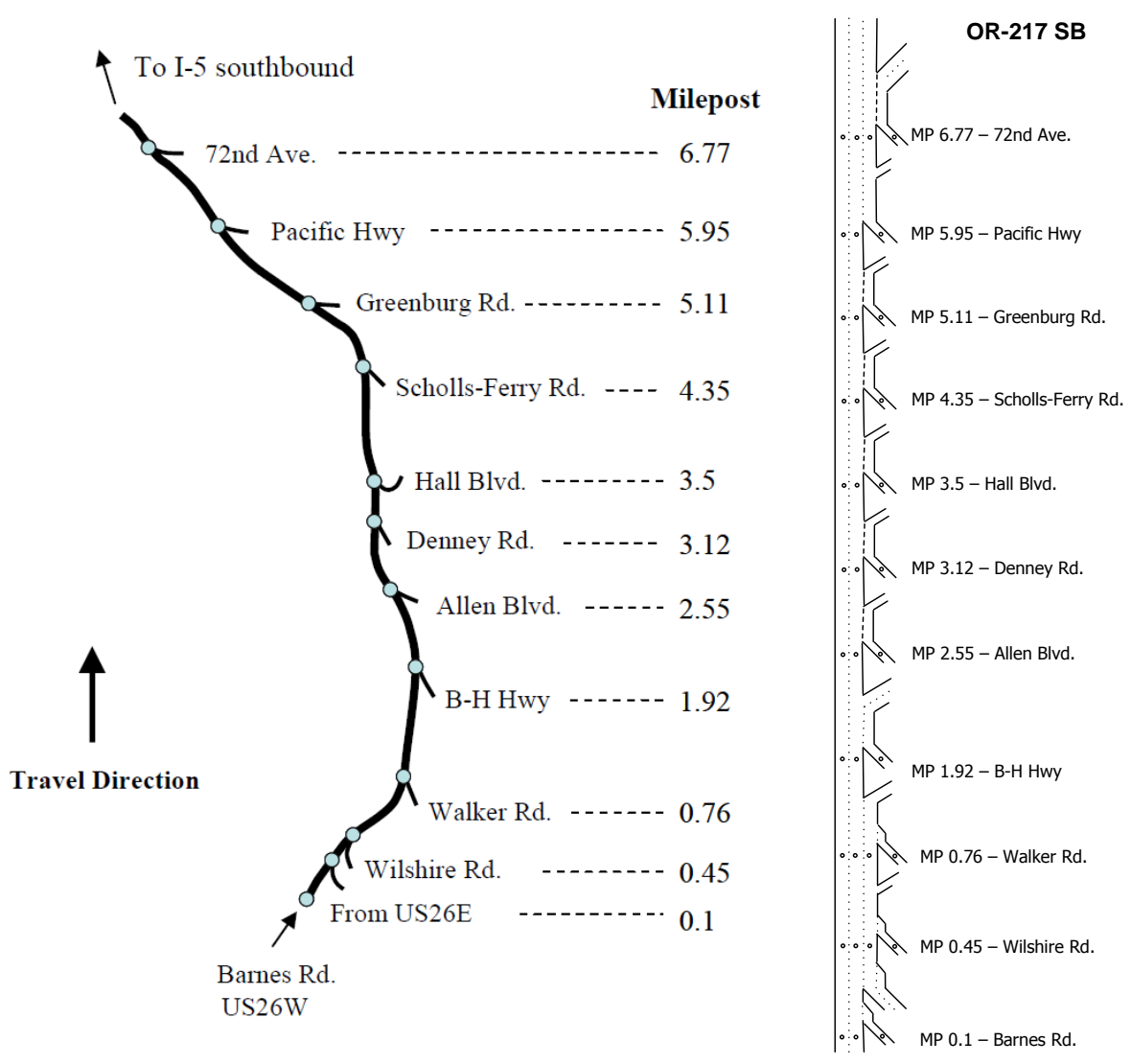

Figure 3.2. Map of ORE 217 Southbound, Portland, OR (Ahn et al., 2007) 


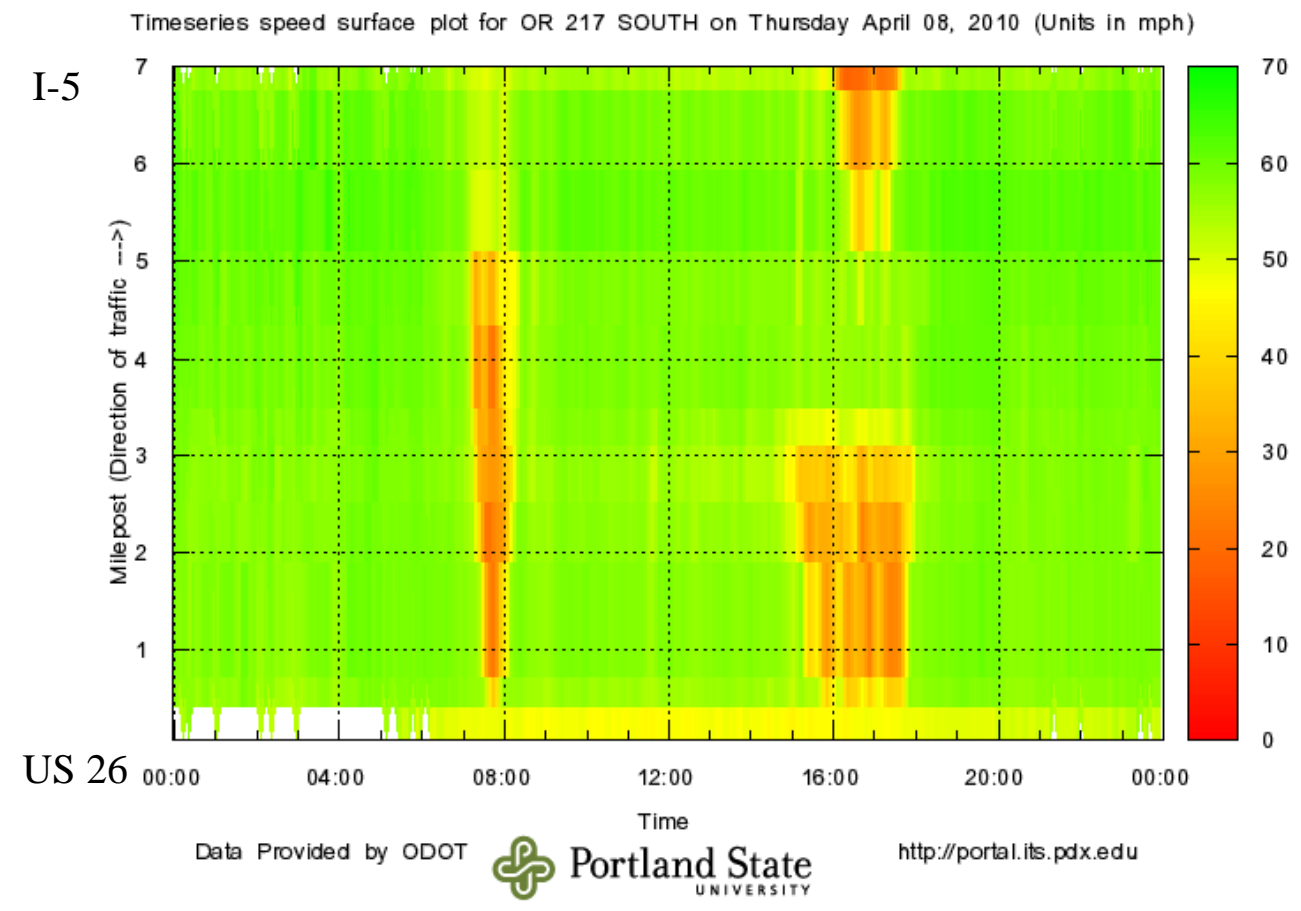

Figure 3.3. Speed Map of ORE 217 Southbound, Portland, OR on Thursday, April 8, 2010

The study area includes mileposts 1.92 (Beaverton-Hillsdale Hwy) and 3.12 (Denney Rd). At both locations the freeway has two main lanes. At milepost 1.92, a two-lane off-ramp to Allen Blvd is located 0.25 mile downstream of the Beaverton-Hillsdale Hwy on-ramp. At milepost 3.12, a one-lane off-ramp to Hall Blvd is located 0.32 mile downstream of the Denney Rd on-ramp. Figure 3.4 shows a sketch of the study locations. The data analysis to be presented in later sections results is for these two locations. 


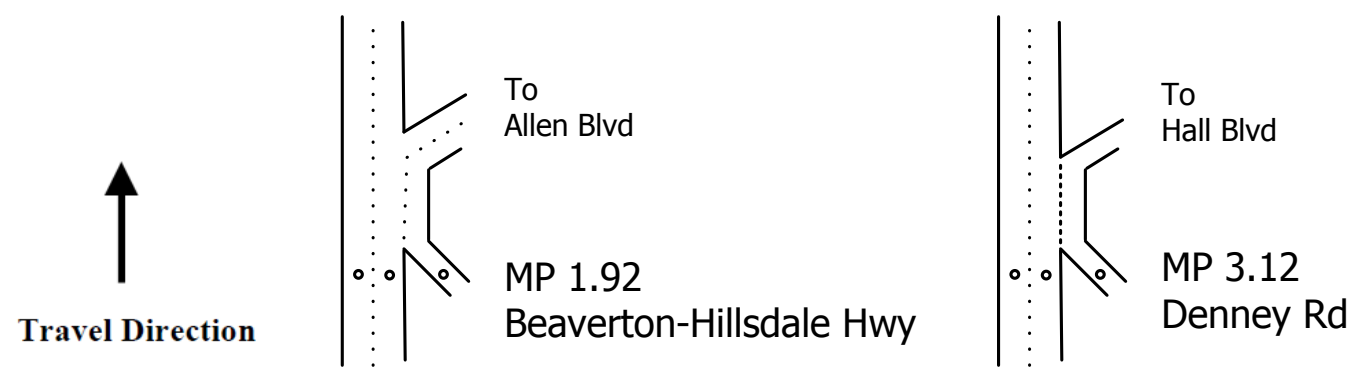

Figure 3.4. Schematic map of the study locations: milepost 1.92 (BeavertonHillsdale Hwy) and milepost 3.12 (Denney Rd)

\subsection{Data}

The data used in this study are from Portland Oregon Regional Transportation Archive Listing (PORTAL: http://portal.its.pdx.edu/). In partnership with the Oregon Department of Transportation (ODOT), PORTAL archives data from more than 670 inductive loop detectors that compose the Portland region's advanced traffic management system (ATMS) (one detector station per segment, segment boundaries established at the midpoints between detectors). These detectors were initially deployed as part of a comprehensive ramp metering system. Therefore, dual mainline loops are located just upstream of on-ramp locations, and the on-ramps themselves are also instrumented. The mean detector spacing is approximately 1 mile. At 20second intervals, each loop detector records vehicle count, average speed of 
these vehicles, and occupancy, or percentage of the sample period when a vehicle was over the detector (Bertini et al., 2005). PORTAL uses the standard midpoint algorithm to generate travel time estimates from 20-second time mean speed data. For this study, archived loop detector data aggregated over 20-second intervals for 30 weekdays from September 2009 to February 2010 are used (see appendix A). Adverse weather can add noise to the speed-flow data. The impact of weather on freeway traffic operations have been widely studied in the literature (Saberi and Bertini, 2010; Rakha et al., 2008; Agarwal et al., 2006; Kyte et al., 2001). To remove variations in speed-flow data due to adverse weather conditions and to be consistent with previous speed-flow studies, we have limited the analysis to days with no adverse weather conditions (i.e. days with no rain or snow). Also only days with good data quality and no major incidents are selected. The analysis is for morning peak periods (6 a.m. to 10 a.m.).

\subsection{Segmentation of Congested and Uncongested Regimes}

Speed-flow relationships for planning applications require v/c ratio to estimate speeds. The $\mathrm{v} / \mathrm{c}$ ratio represents traffic conditions. If $\mathrm{v} / \mathrm{c}<1$ then the traffic condition can be considered "uncongested" and if v/c $\geq 1$ then the traffic 
condition can be considered "congested." To examine speed-flow models with field data recorded by loop detectors, segmentation of congested and uncongested data points is necessary because traffic counts recorded by loop detectors do not measure demand volume during congested conditions. Thus in the absence of vehicle trajectories, queuing theory can be used when demand exceeds capacity. With a spatio-temporal analysis, using data from series of loop detectors upstream of a bottleneck up to the end of the queue, a queuing graph can be created for further queuing analysis (Cassidy and Windover, 1995; Daganzo, 2007). As mentioned earlier, this study empirically evaluates the speed-flow relationships for $\mathrm{v} / \mathrm{c}<1$ using field data. For congested conditions $(\mathrm{v} / \mathrm{c}>1)$ a theoretical approach is taken.

For segmentation of congested and uncongested regimes, different algorithms have been proposed. Several methods utilize speed thresholdbased algorithms. Chen et al. (2004) uses only vehicle speed to indentify traffic states. Zhang and Levinson (2010) developed a similar algorithm but based on occupancy differentials. The ASDA/FOTO algorithm uses Kerner's threephase traffic theory and speed and flow thresholds to identify different traffic states (Kerner et al., 2004). The rescaled cumulative curve method proposed by Cassidy and Bertini (1999) is another tool to track congested traffic features. A 
recent study by Li and Bertini (2010) has tested the rescaled cumulative curve method with speed thresholds and with ASDA/FOTO rules. Their results indicate that the rescaled cumulative curve with speed thresholds works well when only two traffic states (congested and uncongested) are defined. The Chen algorithm was optimized for use in Portland by Wieczorek et al. (2010). They suggested a speed threshold of $35 \mathrm{mph}$ for Portland freeways. Thus, in order to separate congested and uncongested data points in our analysis, rescaled cumulative plots with a speed threshold of $35 \mathrm{mph}$ using 20-second aggregated data are used.

\subsubsection{Rescaled Cumulative Curve}

Rescaled cumulative (oblique) plots of traffic data are a powerful tool for exploring traffic flow phenomena over time and space. Several analyses of traffic features using transformed curves of cumulative vehicle arrival number versus time and cumulative occupancy versus time measured at freeway detector locations can be found in Cassidy and Windover (1995), Cassidy and Bertini (1999), Cassidy and Mauch (2001), Munoz and Daganzo (2002), and Lindgren et al. (2006). A line of constant slope on an oblique cumulative curve can identify nearly stationary traffic patterns where flow or speed alternate 
between higher and lower rates. The use of an oblique coordinate system magnifies changes in flows or speeds and the times when these changes occurred.

Defining $N(t)$ as the speed at time $t$, then the cumulative speed $N(x, t)$ is defined as:

$$
N(x, t)=\sum_{t_{0}}^{t} N(t)
$$

where $N(t)$ is obtained by taking linear interpolations through the 20 -second speed data so that a curve's slope at time $t$ is the speed at location $x$ at that time (See figure 3.5 (a)) as in Li and Bertini (2010).

An oblique coordinate system can be set by reducing $v_{0} t^{\prime}$ from $N(x, t)$, where $v_{0}$ is an oblique scaling rate and $t^{\prime}$ is the elapsed time from the beginning of the curve (See Figure $3.5(\mathrm{~b})$ ). The time window length is defined as $T L=t_{N}-t_{0} ; t_{0}$ and $t_{N}$ are the beginning and end of the analysis period respectively. The oblique scaling rate can be calculated as follows:

$$
v_{0}=\frac{N\left(x, t_{N}\right)-N\left(x, t_{0}\right)}{t_{N}-t_{0}}
$$

The elapsed time is: 


$$
t^{\prime}=t-t_{0}
$$

Then, the oblique value of speed is:

$$
\Delta(x, t)=N(x, t)-v_{0} t^{\prime}
$$

To interpolate lines of constant slope representing stationary traffic conditions, the oblique value $\Delta(x, t)$ which is the speed deviation from the mean can be used. On an oblique cumulative plot of speed, a local maximum oblique value indicates a time at which a speed reduction occurs, and a local minimum indicates the time at which a speed increase occurs.

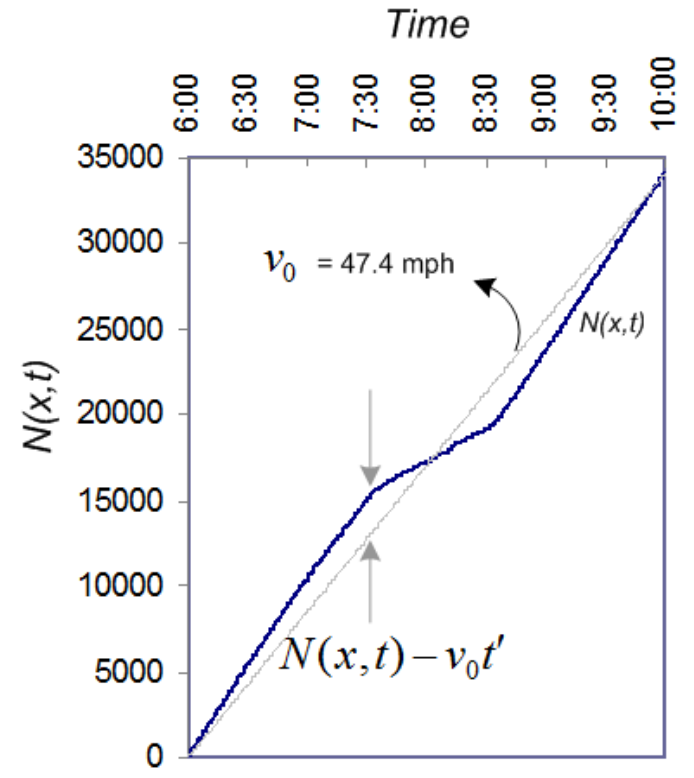

(a)

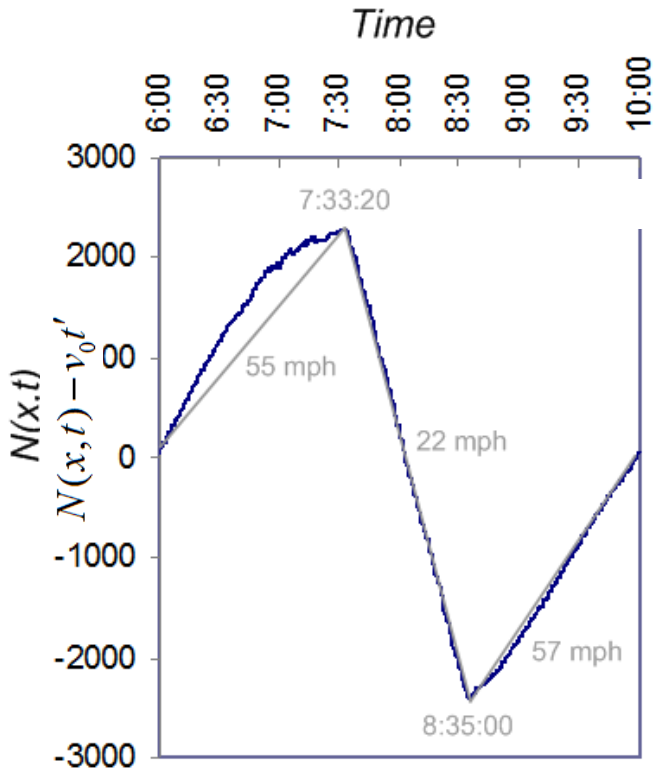

(b)

Figure 3.5. Rescaled cumulative curve construction, ORE 217 SB, milepost 4.35, January 22, 2010 


\subsubsection{Traffic State Identification}

The rescaled cumulative curve method with a speed threshold was suggested by Li and Bertini (2010) for traffic state identification when only two traffic states (congested and uncongested) are defined. From figure 3.5 (b), bottleneck activation and deactivation times can be identified. Between the bottleneck activation and deactivation times, the average speed of $22 \mathrm{mph}$, which is below the speed threshold of $35 \mathrm{mph}$, is observed. Therefore, from 7:33:20 to 8:35:00, the traffic state is congested and for the rest of the time period (from 6:00:00 to 7:33:20 and from 8:35:00 to 10:00:00), the traffic state is uncongested. For the same rescaled cumulative curve constructed before, using 20-second aggregated data, hourly equivalent flow over all lanes versus occupancy averaged over all lanes is plotted in figure 3.6. As can be seen, the fundamental diagram has a clear separation between congested and uncongested regimes with a very few exceptions. 


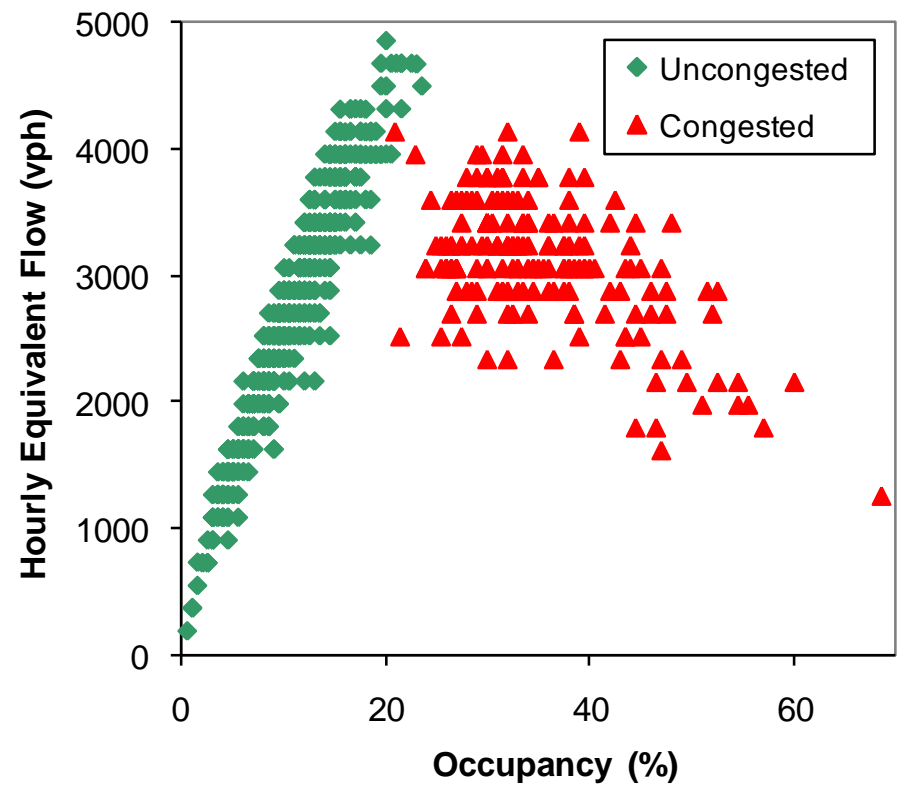

Figure 3.6. Traffic state identification, ORE 217 SB, milepost 4.35, January 22, 2010

\subsection{Parameter Measurement}

\subsubsection{Travel Time Estimation}

For each detector individually, all reported 20-second volume-speed pairs during the study period were used. Data points from the congested conditions were removed. All volumes were then multiplied by a factor of 180 to convert the reported 20 -second counts to equivalent hourly flows. A travel time rate ( $\mathrm{min} / \mathrm{mile})$ was determined for each data point by inversing the reported speed. Because the 20-second volume figures are integer values, the equivalent hourly rates are integer numbers multiples of 180 . The resulting 
flow - travel time pairs can therefore be sorted into about sixteen 'bins' based on these different hourly flows. For each of these bins, the average of the reported travel times was calculated and plotted. For each day, flows were divided by measured "capacity", which is defined as the breakdown flow of that day, to allow presentation of the data in a volume to capacity ratio form. Figure 3.7 shows a sample of equivalent hourly flows versus average travel times plus/minus one standard deviation of travel times for the left lane at milepost 1.92 on January 25th, 2010. Associated travel time distributions as a function of volume are included in Appendix C.

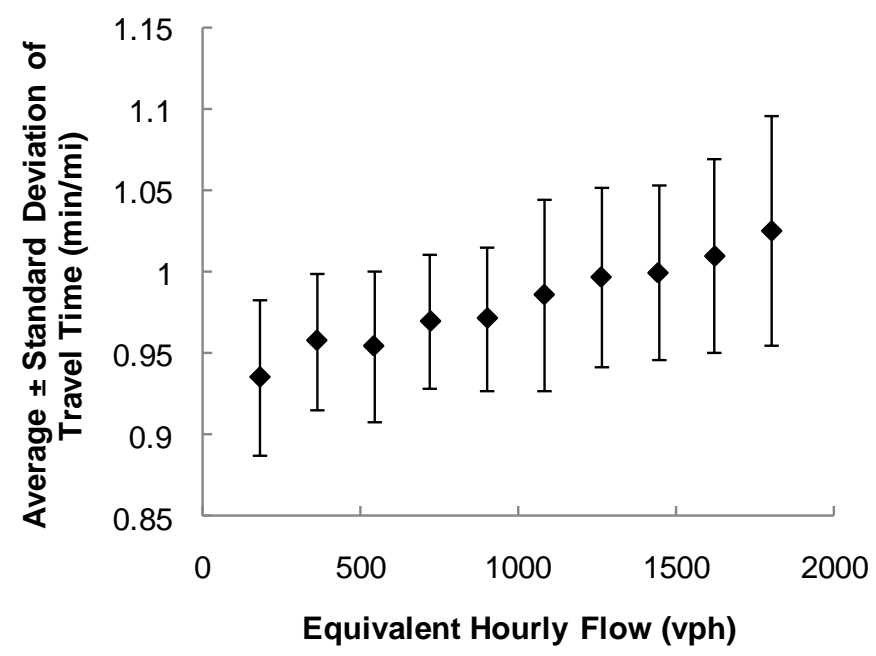

Figure 3.7. Equivalent hourly flows versus average travel times plus/minus one standard deviation of travel times for left lane at milepost 4.35 , on January 22th, 2010. 


\subsubsection{Capacity Measurement}

For each detector on each day, the apparent maximum flow before breakdown and breakdown flow using 5-minute and 15-minute aggregated data were measured. 5-minute and 15-minute aggregation levels are used to be consistent with the literature and HCM definition. Figure 3.8 shows a sample time-series of averages of flow and speed aggregated over 5-minute intervals on the left lane at milepost 1.92 on December 10th, 2009. The maximum observed flow before breakdown is $1,944 \mathrm{vph}$ while the breakdown flow occurs at 1,536 vph. Breakdown points are identified when the speed drop between two consecutive time intervals exceeds a threshold of $10 \mathrm{mph}$ and low speed (lower than $55 \mathrm{mph}$ ) is sustained for some time as defined in Dong and Mahmassani (2010). 


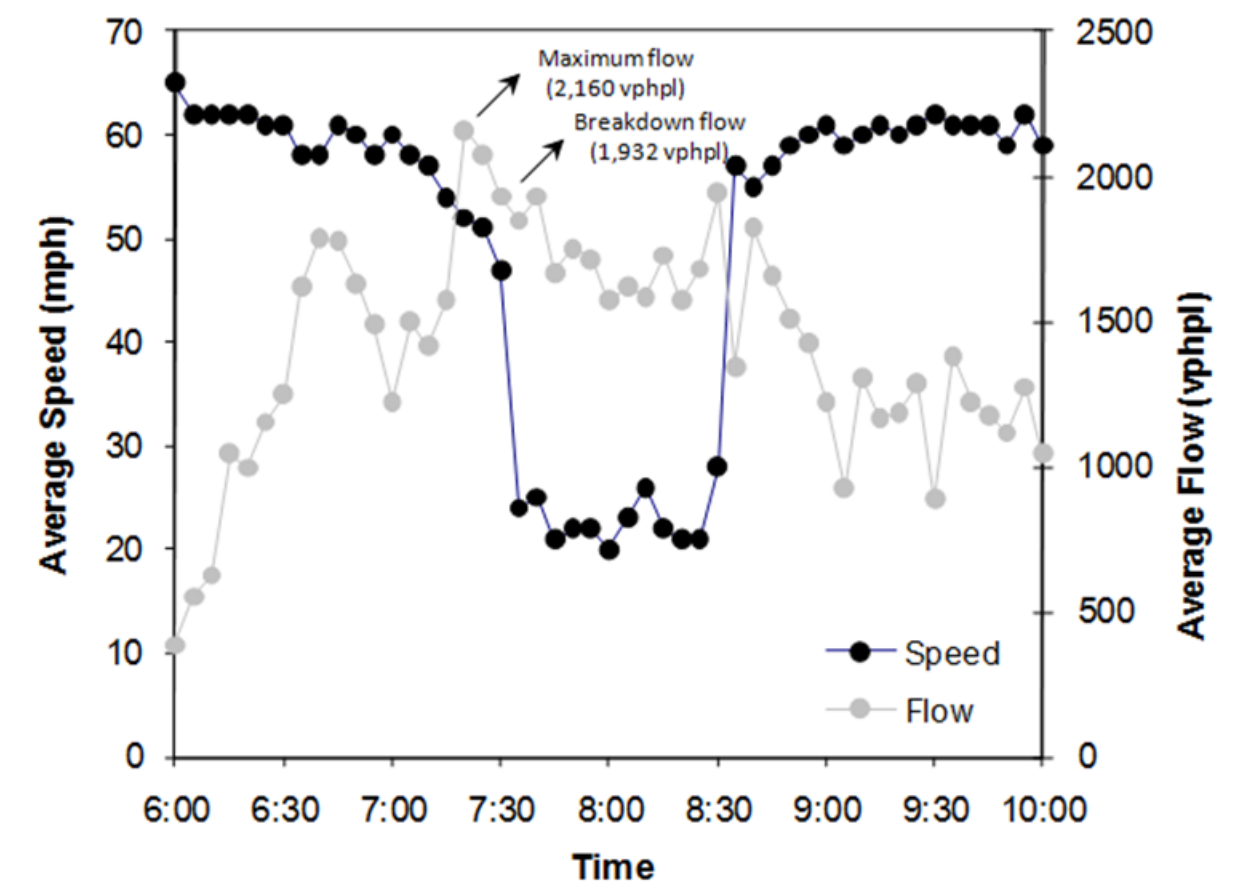

Figure 3.8. Time-series of average flows and speeds for left lane at milepost 4.35 , on January 22th, 2010.

\subsubsection{Free-Flow Speed Measurement}

HCM (2000) defines free-flow speed as "the mean speed of passenger cars under low to moderate flow rates that can be accommodated on a uniform roadway under prevailing roadway and traffic conditions." For each detector on each day, the average speed under equivalent hourly flow rates equal and smaller than 360 vph was calculated as free-flow speed.

\subsection{Quantile Function}

The behavior of a random variable (e.g. capacity) can be characterized by its probability distribution. The cumulative distribution function (CDF) 
describes the probability distribution of a real-valued random variable $\mathrm{X}$. For any real number $\mathrm{x}$, the $\mathrm{CDF}$ is expressed as Equation 3.4. CDF represents the probability that the random variable $X$ takes on a value less than or equal to $x$.

$$
F_{X}(x)=P(X \leq x)=p
$$

A quantile function of a probability distribution is the inverse of its cumulative distribution function. Generally, a distribution does not have an inverse. Thus, for $p \in[0,1]$, the quantile function is expressed as:

$$
F^{-1}(p)=\inf _{x \in R}\{F(x) \geq p\}
$$

For a probability $0<p<1$, the quantile function returns the minimum value of $x$ for which the Equation 3.5 holds. Quantile functions are widely used in statistical applications and Monte Carlo simulations. Based on the measured capacities at each location, the empirical CDF and quantile function of capacity will be developed and discussed later in chapter 4 . The quantile function will be used as a tool to take into account the stochastic nature of capacity when modeling the travel time and volume to capacity ratio relationship. 


\subsection{DATA ANALYSIS}

This chapter will present the results of the data analysis. In the first section, measured free-flow speed, speed at capacity and capacity are analyzed. The second section presents the results of the goodness of fit tests. In the third section, results of the calibration of different models are presented. The fourth section analyses capacity. In the fifth section, a summary of key findings are presented.

\subsection{Measured Free-Flow Speed, Speed at Capacity, and Capacity}

Poor choices of free-flow speed, capacity, and speed at capacity can seriously compromise the accuracy of the speed-flow models. Therefore, before performing the goodness of fit tests, free-flow speed, capacity (defined as 5-minute breakdown flow here), and speed at capacity as defined in the previous chapter have been measured. Figures 4.1 to 4.3 show box plots of measured free-flow speed, speed at capacity, and capacity over the selected 30 days at each location ("Lane1" is the left lane and "Lane2" is the right lane in the figures) using 5-minute aggregated data. As can be seen in figure 4.1, the measured median free-flow speed in the left lane is $4 \mathrm{mph}$ larger than the measured median free-flow speed in the right lane at both locations. Results of 
t-tests show that the observed differences between lanes are statistically significant at the $99 \%$ level.

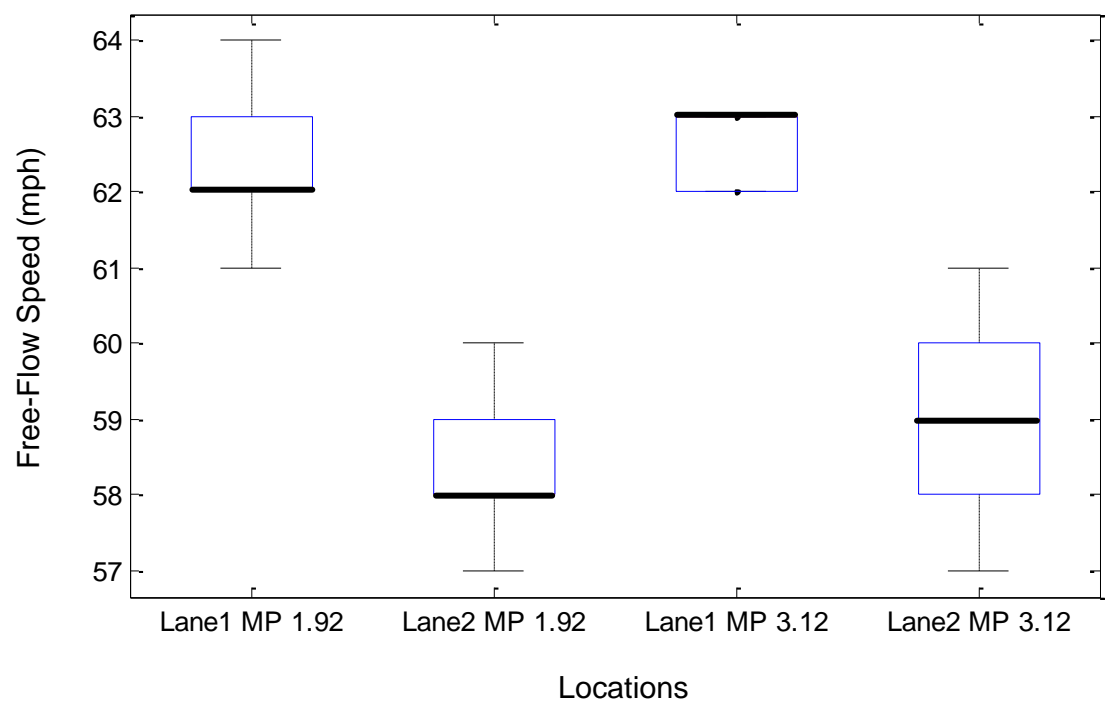

Figure 4.1. Box plots of free-flow speed

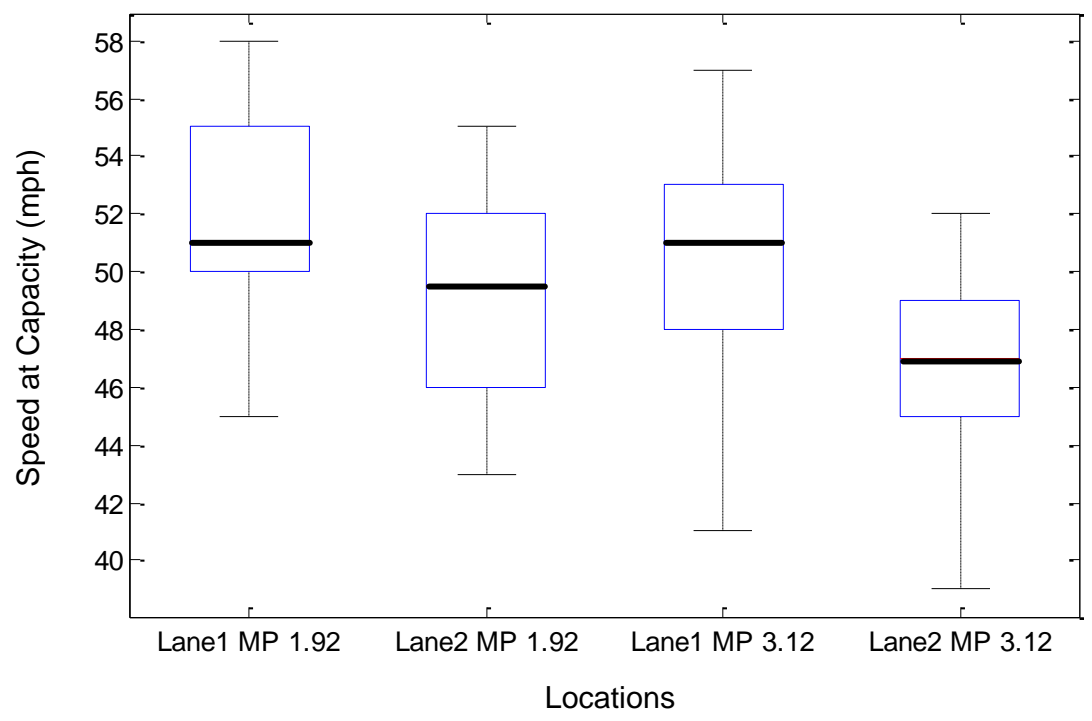

Figure 4.2. Box plots of speed at capacity 
It can be seen in figure 4.2 that the measured median speed at capacity in the left lane is $2-4 \mathrm{mph}$ larger than the measured median speed at capacity in the right lane. Results of t-tests show that the observed differences between lanes are statistically significant at the $95 \%$ level. Also it is observed that the measured median speed at capacity is 9-12 mph lower than the measured median free-flow speed.

Figure 4.3 shows the box plots of measured capacity (5-minute breakdown flows) for each lane. The figure clearly shows the difference between measured capacities at each lane. Results of t-tests show that the observed differences between lanes are statistically significance at the $99 \%$ level. For both of the locations, the left lane has a higher measured capacity compared to the right lane. 


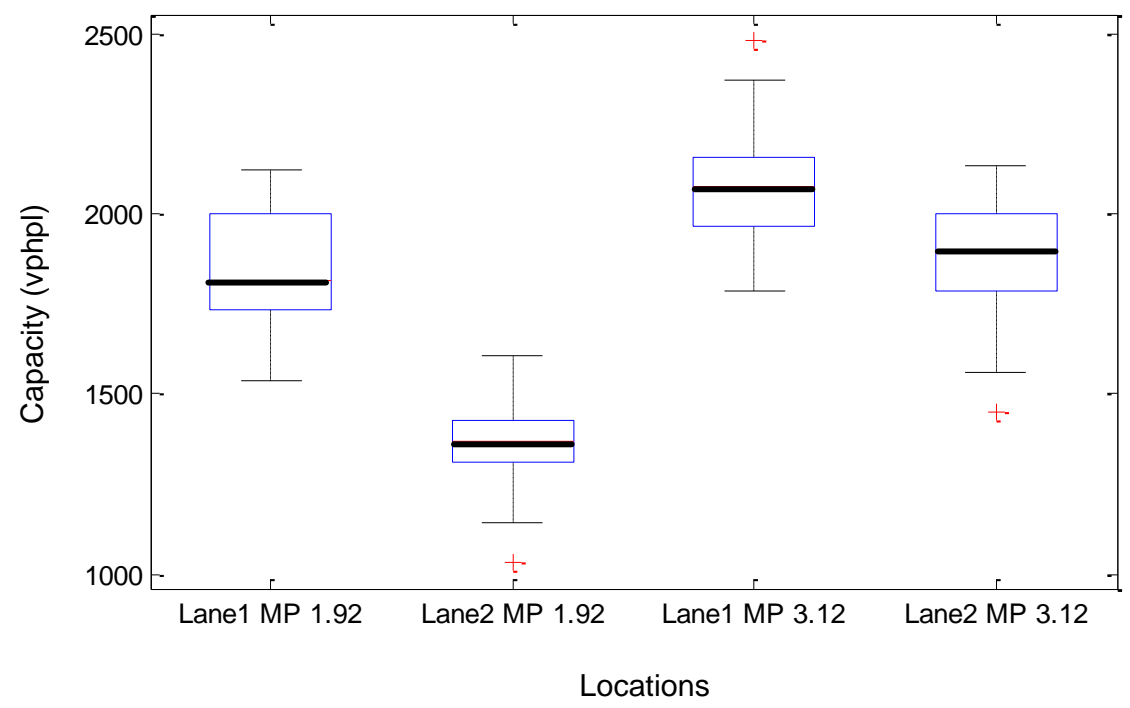

Figure 4.3. Box plots of capacity

It is observed that the right lane at milepost 1.92 has the lowest measured capacity among all the lanes which is possibly due to the lane characteristics.

\subsection{Goodness of Fit Tests}

In this section the travel time estimates produced by the standard BPR, METRO updated BPR, METRO conical, Akçelik, and HCM 2000 speed-flow models are compared with real-world data (travel times estimation from loop detector data) for v/c ratios below 1. Figures 4.4 to 4.7 show the estimated travel time vs. v/c ratio curves with field data for $v / c<1$ at each study location and in each lane. As can be seen, all the travel time vs. v/c ratio curves except 
the conical function fall closely into the cloud of the field data. The median value of 5-minute breakdown flow at each location is used as capacity in calculating the $\mathrm{v} / \mathrm{c}$ ratios.

The next step is to test goodness of fit of the various equations to the data. A statistical comparison of the models at each location is presented in Table 4.1. This table shows the parameters used in each model along with the bias, root mean square error (RMSE), and mean absolute error (MAE) for each equation when compared against the observed data. Bias, RMSE, and MAE are calculated as follows:

$$
\begin{gathered}
\text { Bias }=\frac{\sum_{i}^{n}\left(\hat{t}_{i}-t_{i}\right)}{n} \\
\text { RMSE }=\sqrt{\frac{\sum_{i}^{n}\left(\hat{t}_{i}-t_{i}\right)^{2}}{n}} \\
\text { MAE }=\frac{\sum_{i}^{n}\left|\hat{t}_{i}-t_{i}\right|}{n}
\end{gathered}
$$

where $\hat{t}_{i}$ is the estimated value of travel time by the model, $t_{i}$ is the actual travel time value, and $n$ is the total number of observations.

For the left lane at milepost 1.92, the standard BPR function has the lowest RMSE and MAE. For this location, the Akçelik function has the lowest 
Bias. For the left lane at the same milepost, the updated BPR by METRO has the lowest RMSE and MAE while the HCM 2000 function has the lowest Bias.

For the left lane at milepost 3.12, similar to the left lane at previous milepost, the standard BPR function has the lowest RMSE and MAE while the Akçelik function has the lowest Bias. For the left lane at the same milepost, the standard BPR function has the best overall performance of the equations tested. It has the lowest Bias, RMSE, and MAE. 


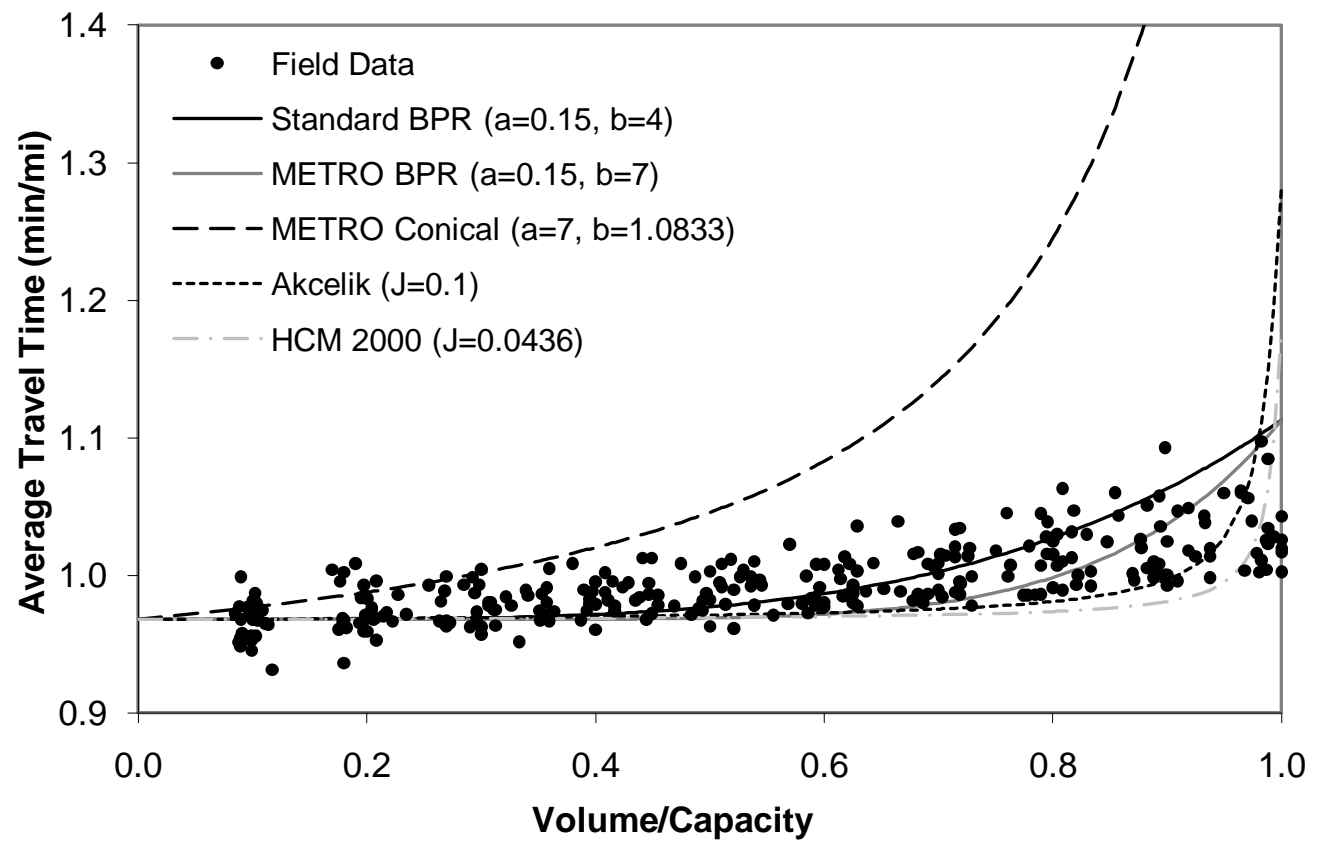

Figure 4.4. Comparison of travel time estimation functions against field data for $v / c<1$ for the left lane at milepost 1.92

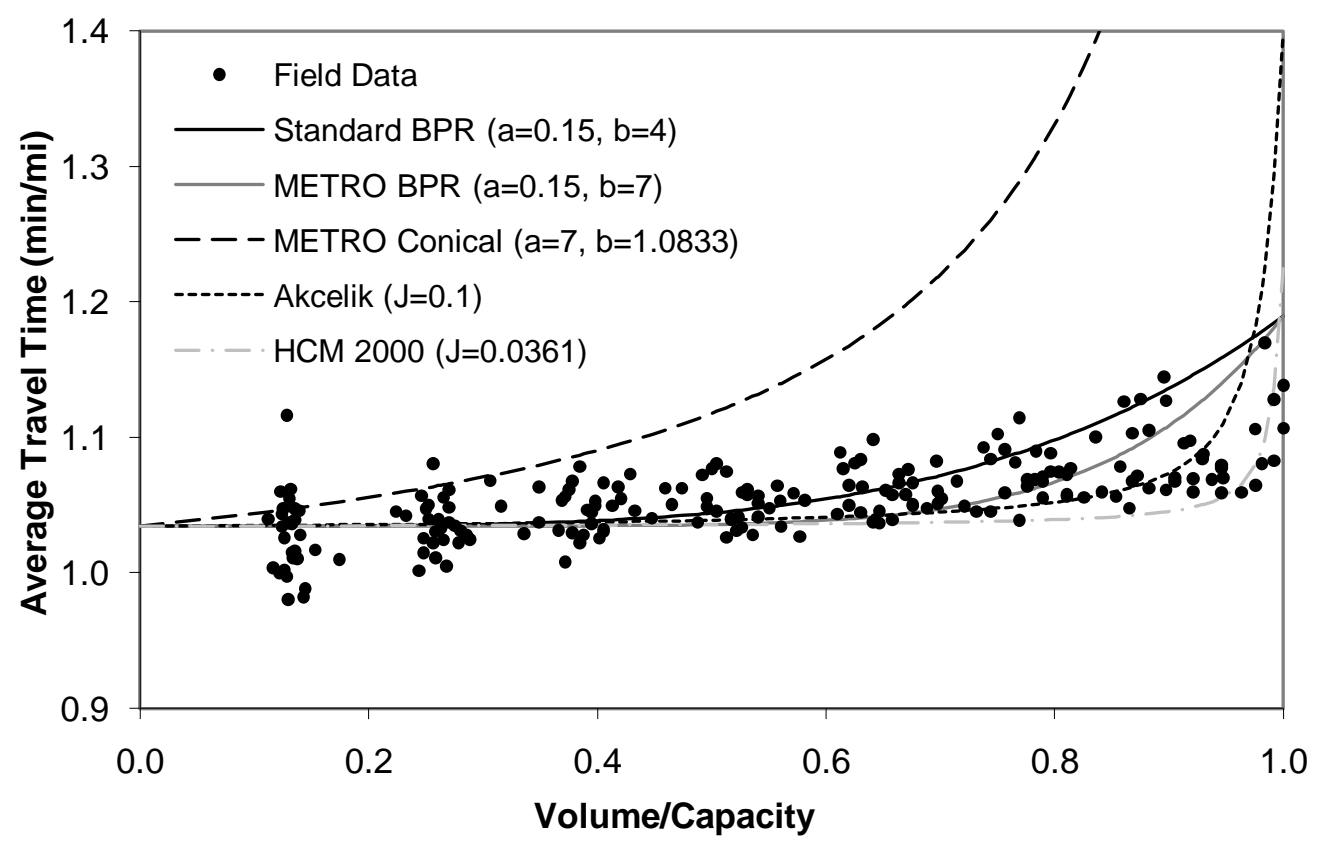

Figure 4.5. Comparison of travel time estimation functions against field data for $v / c<1$ for the right lane at milepost 1.92 


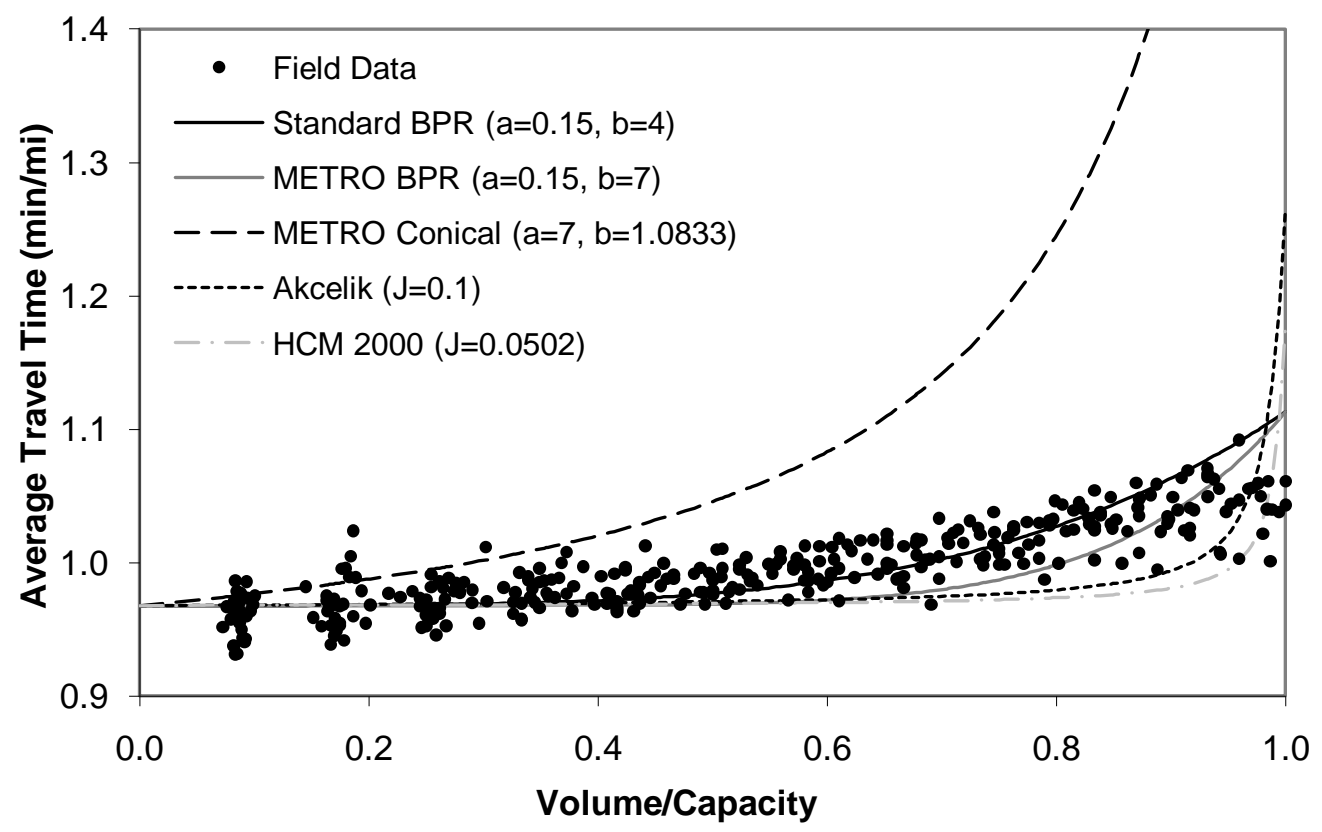

Figure 4.6. Comparison of travel time estimation functions against field data for $v / c<1$ for the left lane at milepost 3.12

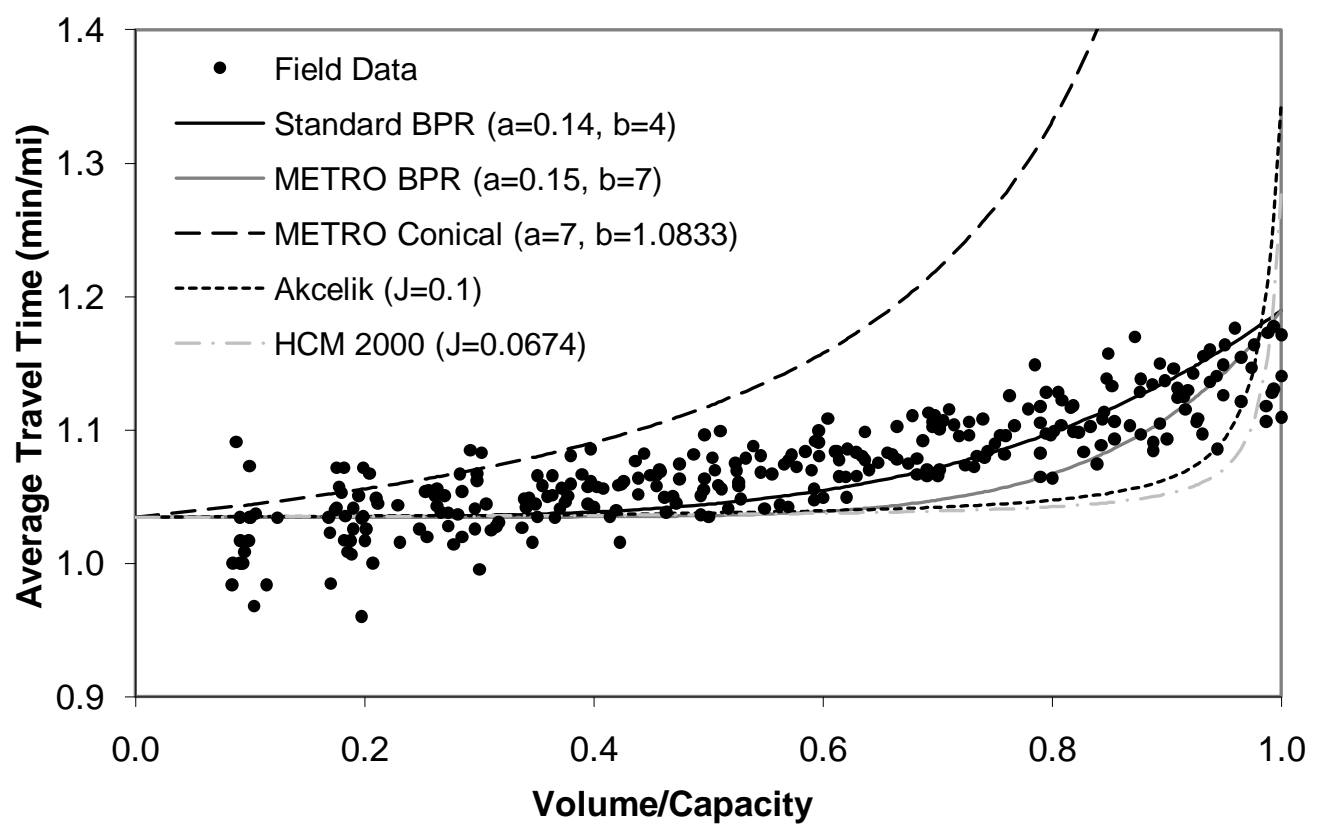

Figure 4.7. Comparison of travel time estimation functions against field data for $v / c<1$ for the right lane at milepost 3.12 


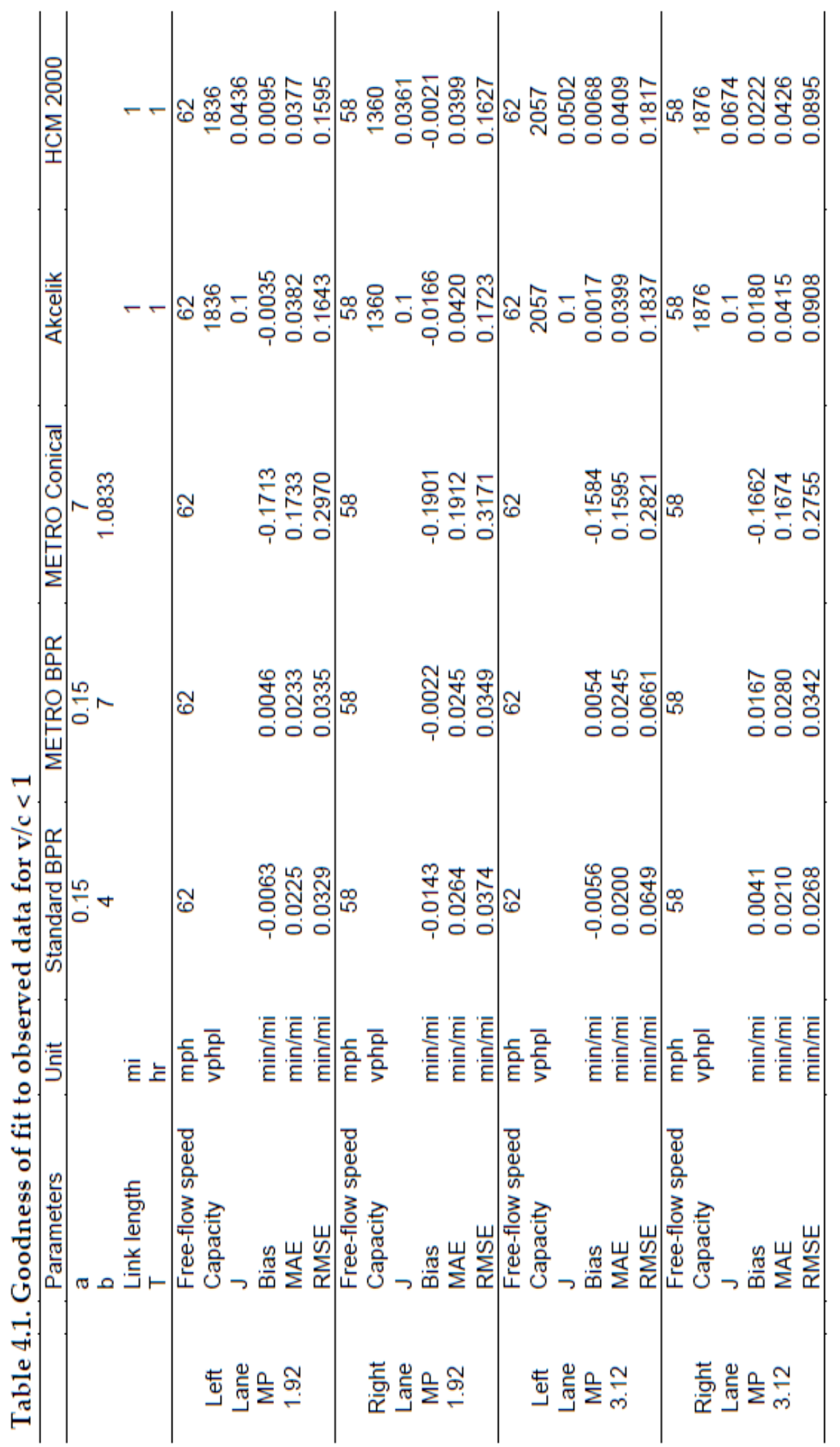


To explore the possible trend in errors, residuals are plotted.

Residual is the difference between the observed value of the desired variable (travel time here) and the value estimated by the model $\left(\hat{t}_{i}-t_{i}\right)$. The residuals can be used to visualize the distribution of bias. Therefore, if the residuals appear to behave randomly, it suggests that the model fits the data well. However, if the residuals display a systematic pattern, it is a clear sign that the model fits the data poorly.

Figures 4.8 to 4.11 show the residual values for each model at each location and in each lane (note that the y-scales are different). As can be seen, for all of the models, a systematic error can be observed. For the standard BPR function, for $\mathrm{v} / \mathrm{c}<0.8$, the residuals appear randomly scattered around zero indicating that the model describes the data well. However, for v/c between 0.8 and 1, the residuals are systematically negative for much of the data range indicating that this model is a poor fit for the data. The METRO updated BPR, Akçelik, and HCM 2000 functions behave similarly for v/c ratios close to 1. A systematic error for smaller v/c ratios is also observed in these models. For v/c ratios between 0 and 0.8 , the residuals are increasing positively. For the conical function, a different systematic error is observed. The residuals 
increase negatively while the v/c ratio gets larger. Similar trends in residuals are observed at all study locations.
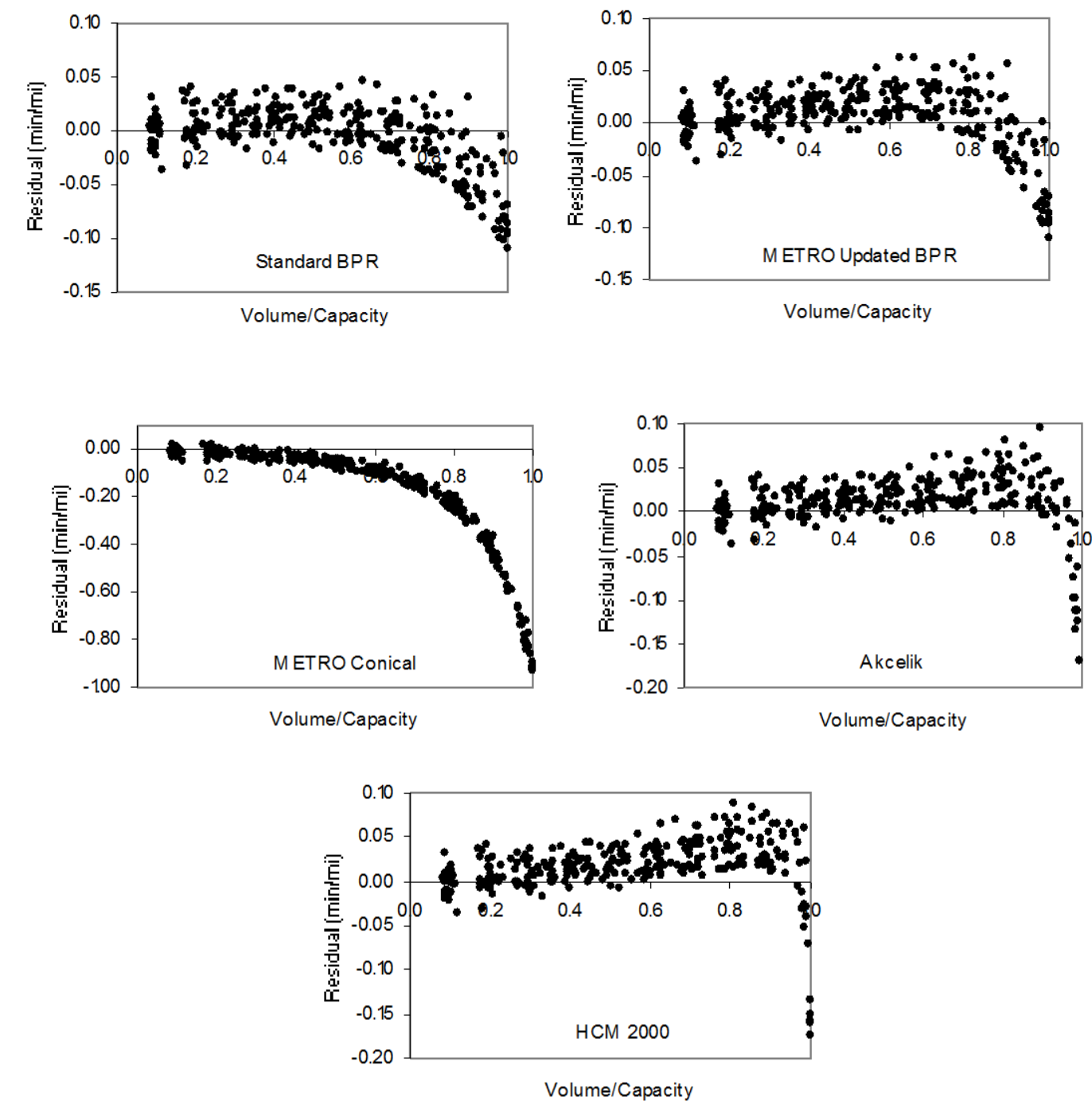

Figure 4.8. Residual values for the tested equations for the left lane at milepost 1.92 

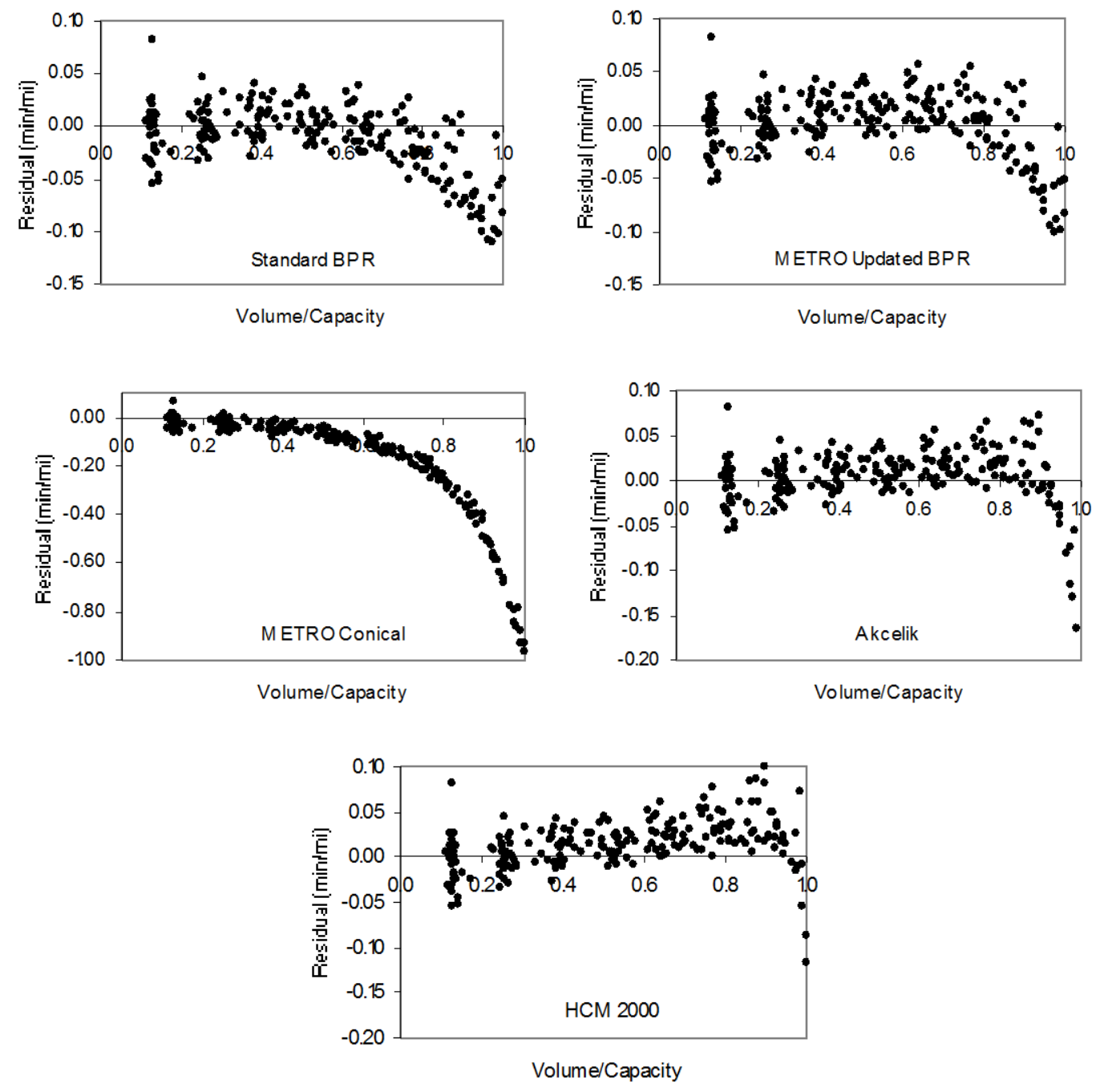

Figure 4.9. Residual values for the tested equations for the right lane at milepost 1.92 

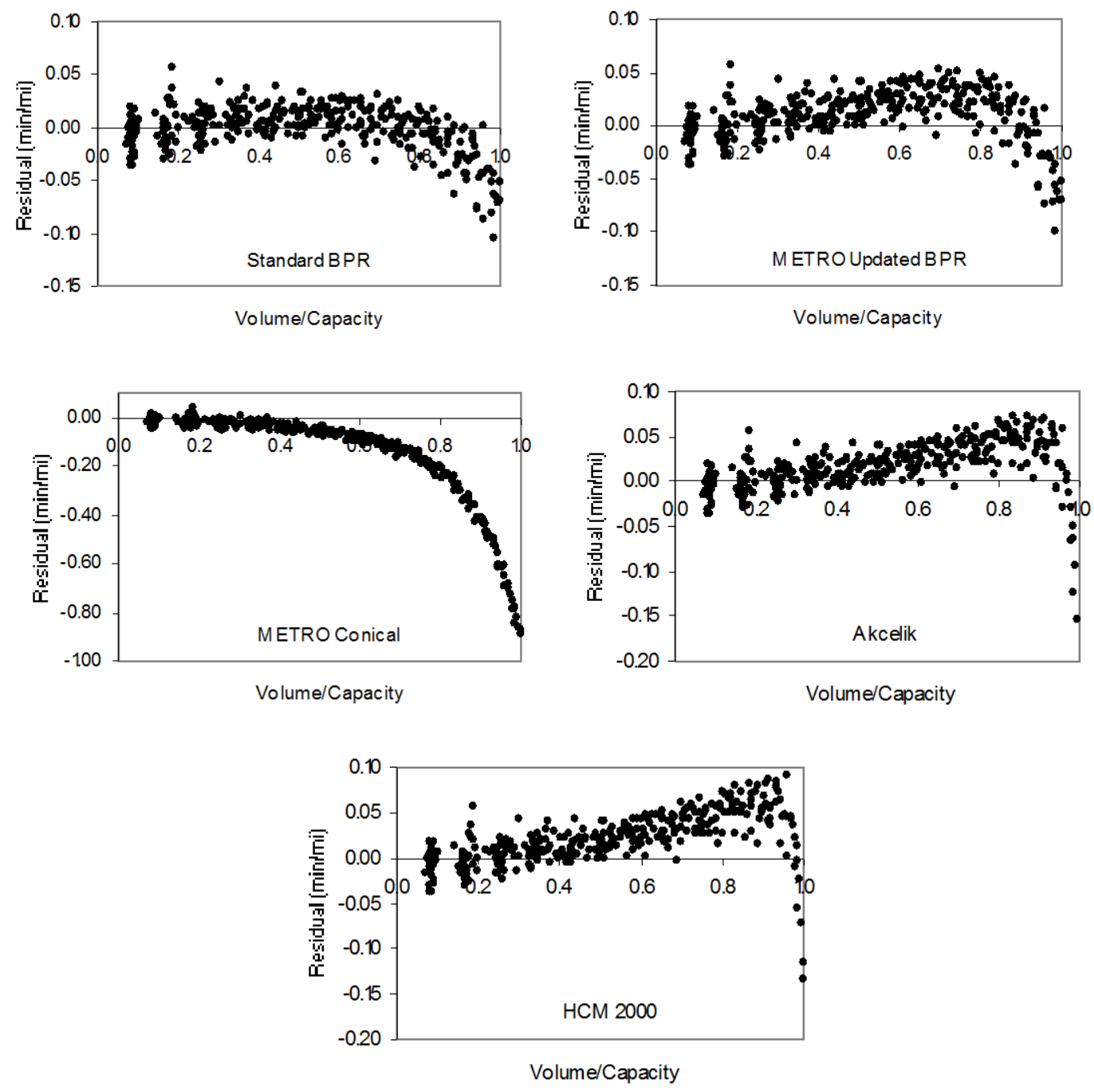

Figure 4.10. Residual values for the tested equations for the left lane at milepost 3.12 

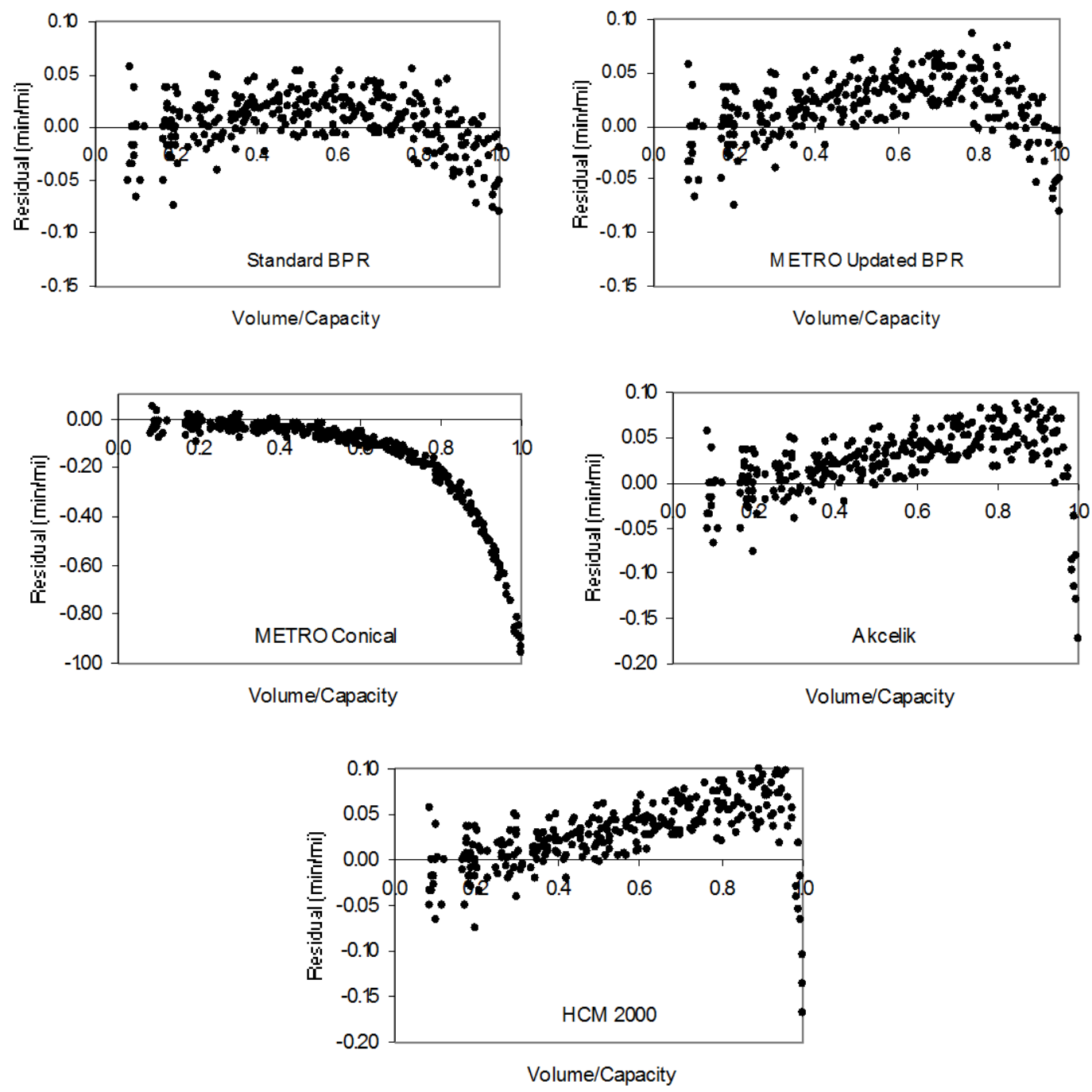

Figure 4.11. Residual values for the tested equations for the right lane at milepost 3.12 
To further explore the goodness of fit of tested models, for each model, bias, RMSE, and MAE are calculated separately for different ranges of $\mathrm{v} / \mathrm{c}$ ratios (See figures 4.12 to 4.14 ). Based on the results from the analysis of residuals, $v / c$ ratio is subdivided into three categories: a) $0 \leq v / c \leq 0.5$; b) $0.5<v / c \leq 0.8$; and c) $0.8<v / c \leq 1$. As can be seen, the absolute bias, RMSE, and MAE for $0.8<v / c \leq 1$ have higher values than the absolute bias, RMSE, and MAE for other v/c ratio ranges for all the tested models at all locations except for the METRO BPR function at the right lane of milepost 3.12. Result confirm what previously observed in residuals that all the tested models work poorly for $\mathrm{v} / \mathrm{c}$ ratios close to 1 compared to smaller $\mathrm{v} / \mathrm{c}$ ratios. 

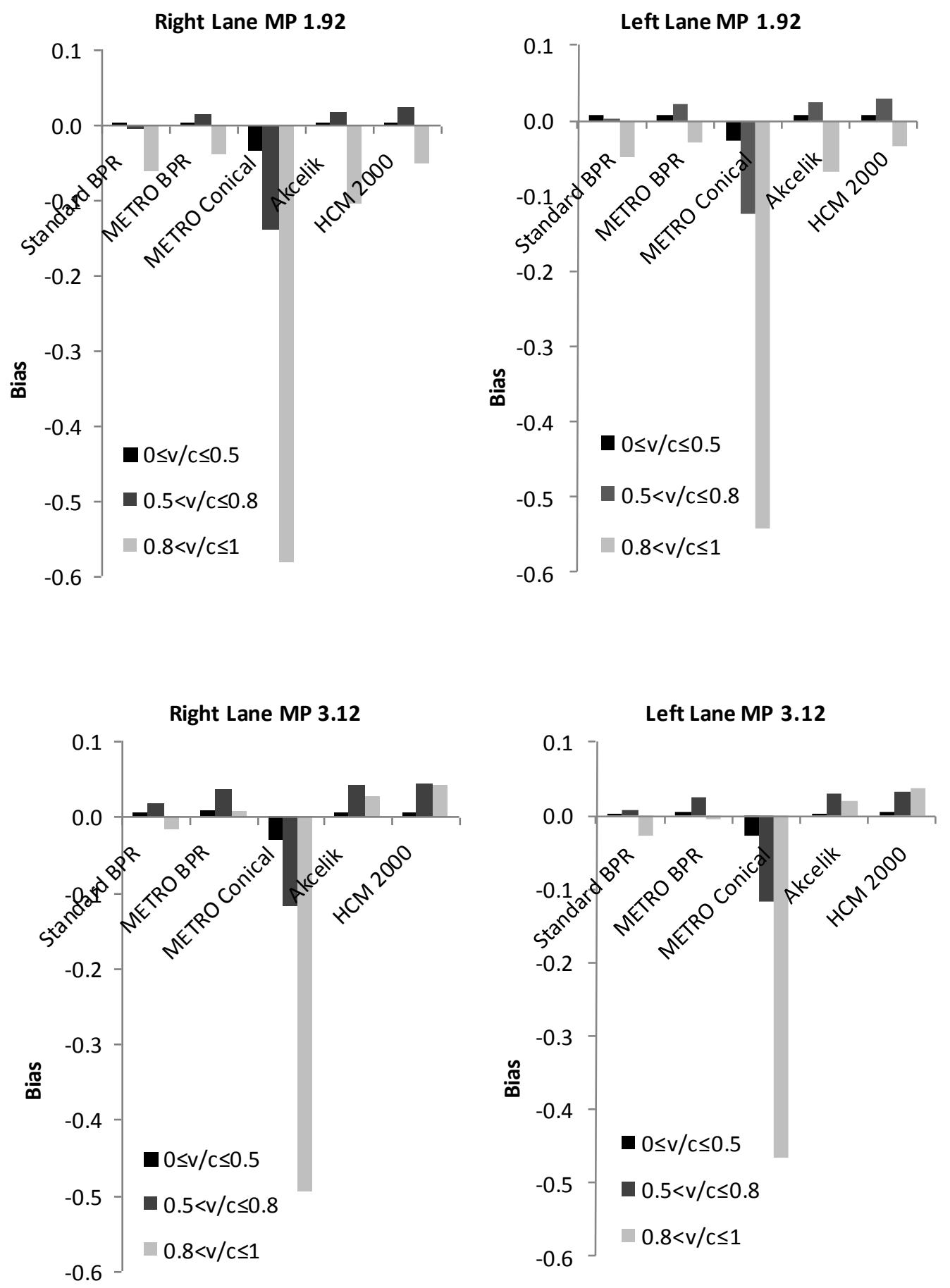

Figure 4.12. Bias values for different ranges of $\mathrm{v} / \mathrm{c}$ ratio 

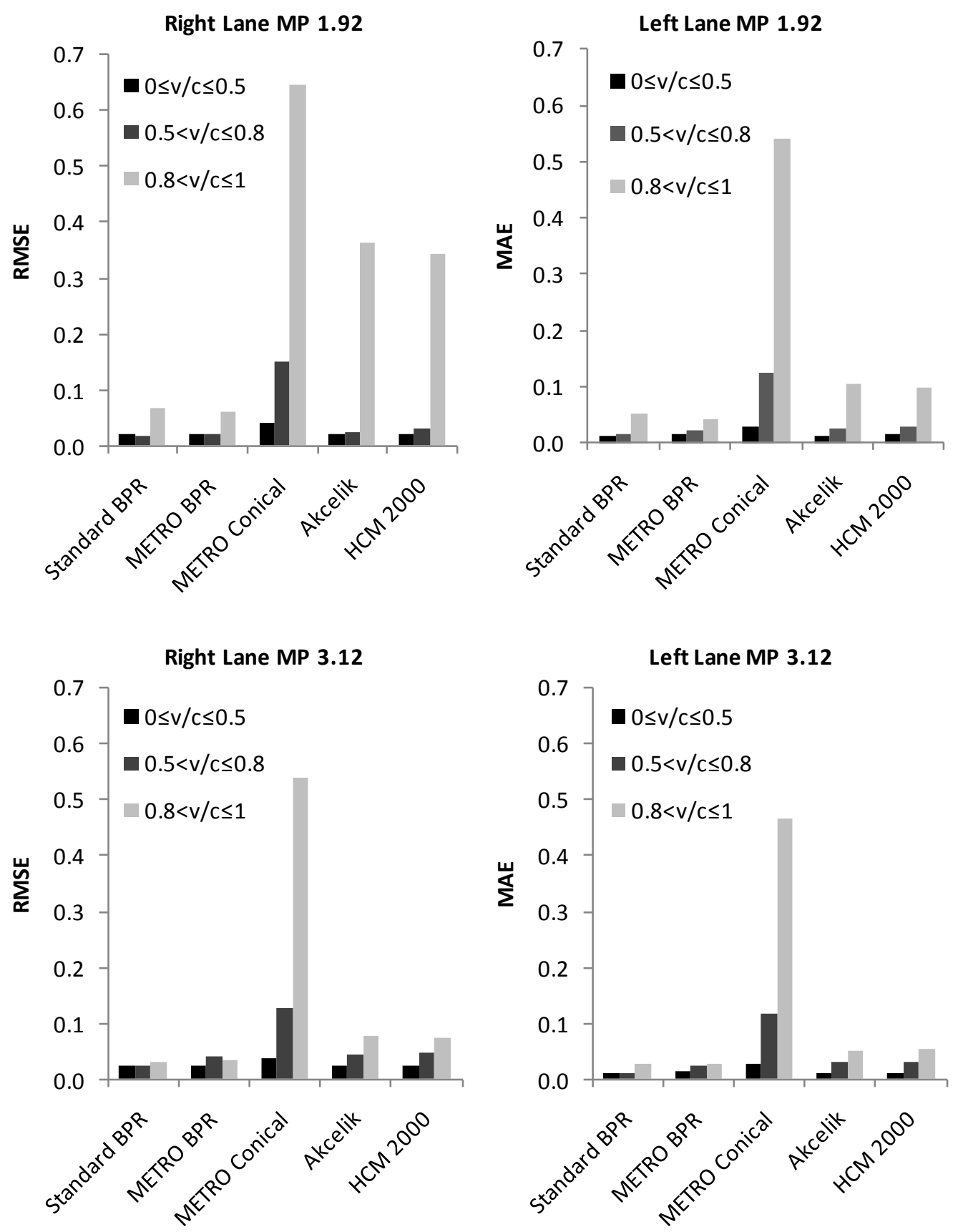

Figure 4.13. RMSE values for different ranges of v/c ratio 

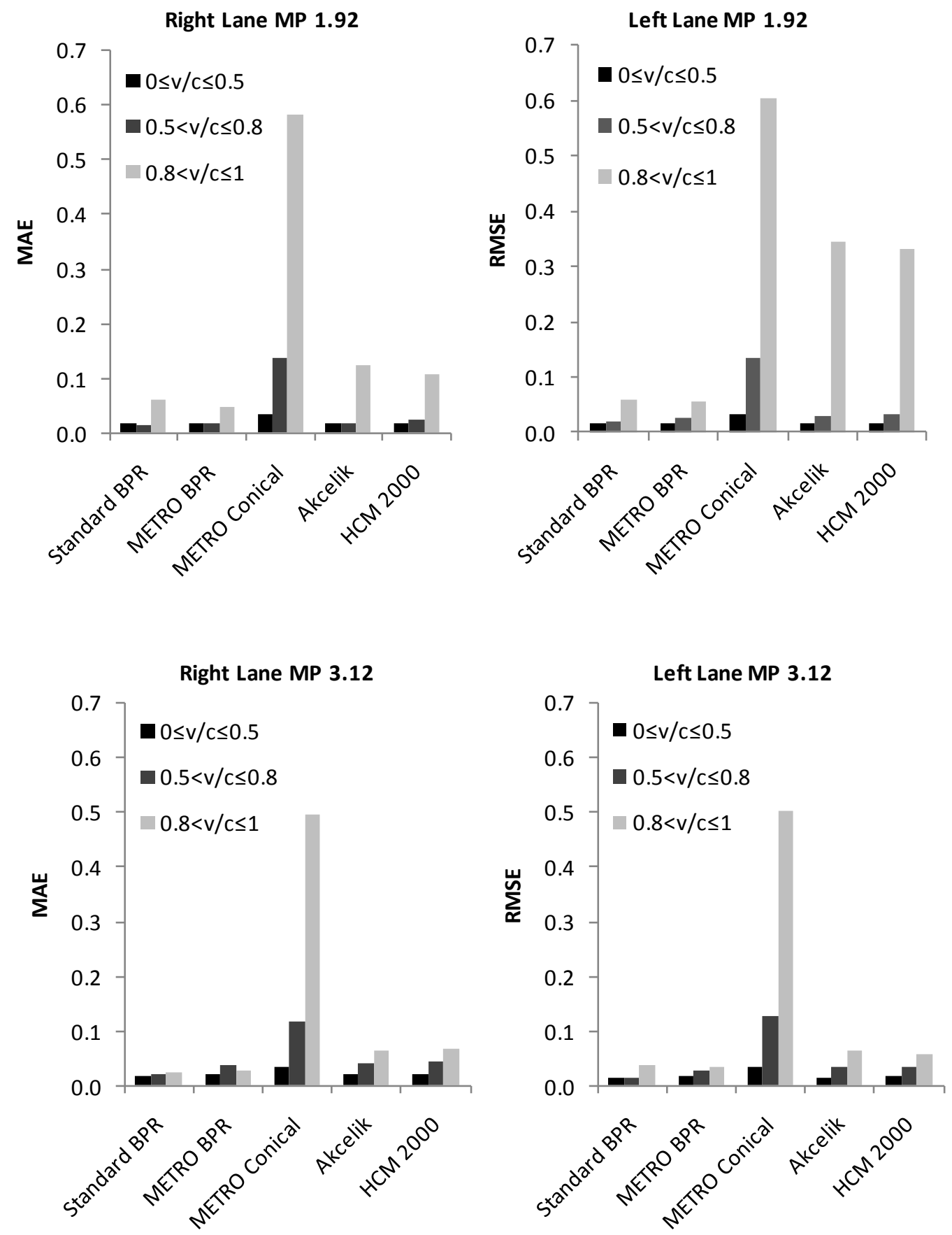

Figure 4.14. MAE values for different ranges of v/c ratio 


\subsection{Calibration}

Model calibration generally consists of changing values of model input parameters in an attempt to match field conditions within some acceptable criteria. Lack of proper site characterization may result in a model that is calibrated to a set of conditions which are not representative of actual field conditions. The data used for calibration here are from days with no adverse weather (no rain or snow) and no major accident at the study locations. Calibration is also performed for each lane separately since the characteristics of the study lanes are different. The objective function of calibration is set to minimize the RMSE as follows:

$$
\text { Minimize } \sqrt{\frac{\sum_{i}^{n}\left(\hat{t}_{i}-t_{i}\right)^{2}}{n}} \text {, }
$$

Table 4.2 shows the calibration results including calibrated input parameters of each model with the measures of goodness of fit after calibration for each study location. For all of the locations, the calibrated BPR function has the best overall performance, with smallest bias and RMSE, of the equations tested while the calibrated conical function has the lowest MAE. Figures 4.15 to 4.18 show estimated travel times by calibrated equations vs. v/c ratio curves with field data for $\mathrm{v} / \mathrm{c}<1$ at each study location and in each lane. 
As can be seen, the calibrated BPR curve fits data better than other plotted curves.

In the BPR function, parameter $a$ determines the ratio of free-flow travel time to the travel time at capacity. The calibrated $a$ is smaller than the $a$ used in the standard BPR function. Parameter $b$ determines how rapidly travel time increases from the free-flow travel time. Smaller values of $b$ makes estimated travel time more sensitive to $\mathrm{v} / \mathrm{c}$ ratio. The calibrated $b$ is also smaller than the $b$ used in the standard BPR function.

For the calibrated conical function, smaller values for parameters $a$ and $b$ are obtained as compared to the values used in the METRO updated function which makes estimated travel time more sensitive to $\mathrm{v} / \mathrm{c}$ ratio. However, the calibrated conical function still has a high RMSE and bias. The conical function assumes that the travel time at capacity is two times greater than the free-flow travel time which may not necessarily be true.

In the Akçelik function, the calibrated parameter $J$ is much smaller than what is originally suggested by Akçelik. Smaller values of $J$ make estimated travel time less sensitive to the $\mathrm{v} / \mathrm{c}$ ratio. This is contrary to the calibration results of the other models. In the calibrated BPR and conical functions, estimated travel time has become more sensitive to v/c ratio while 
the calibrated Akçelik function reveals opposite results. This is because the Akçelik curve for $\mathrm{v} / \mathrm{c}$ ratios smaller than 0.9 is too flat compared to the field data. 


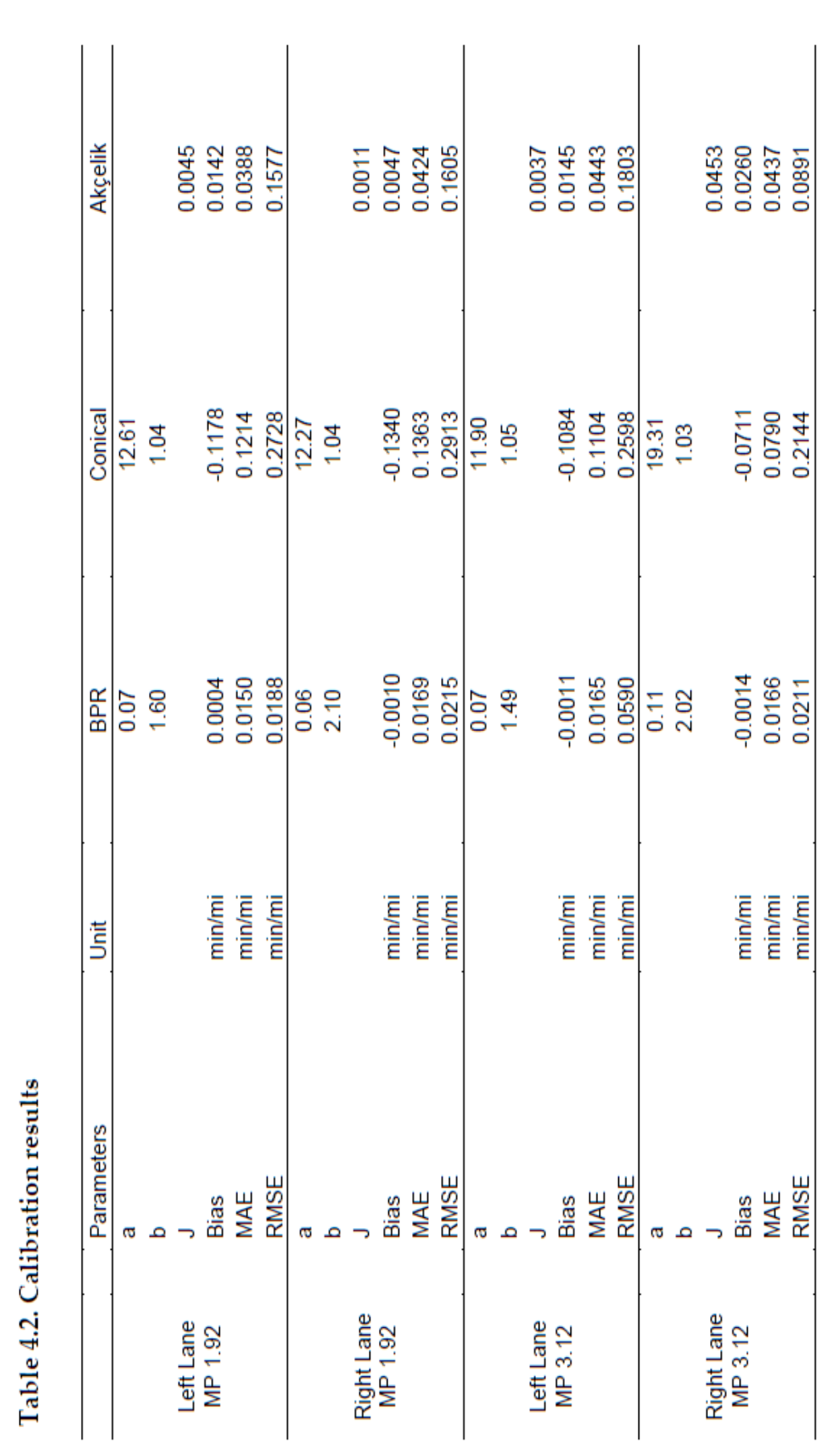

72 


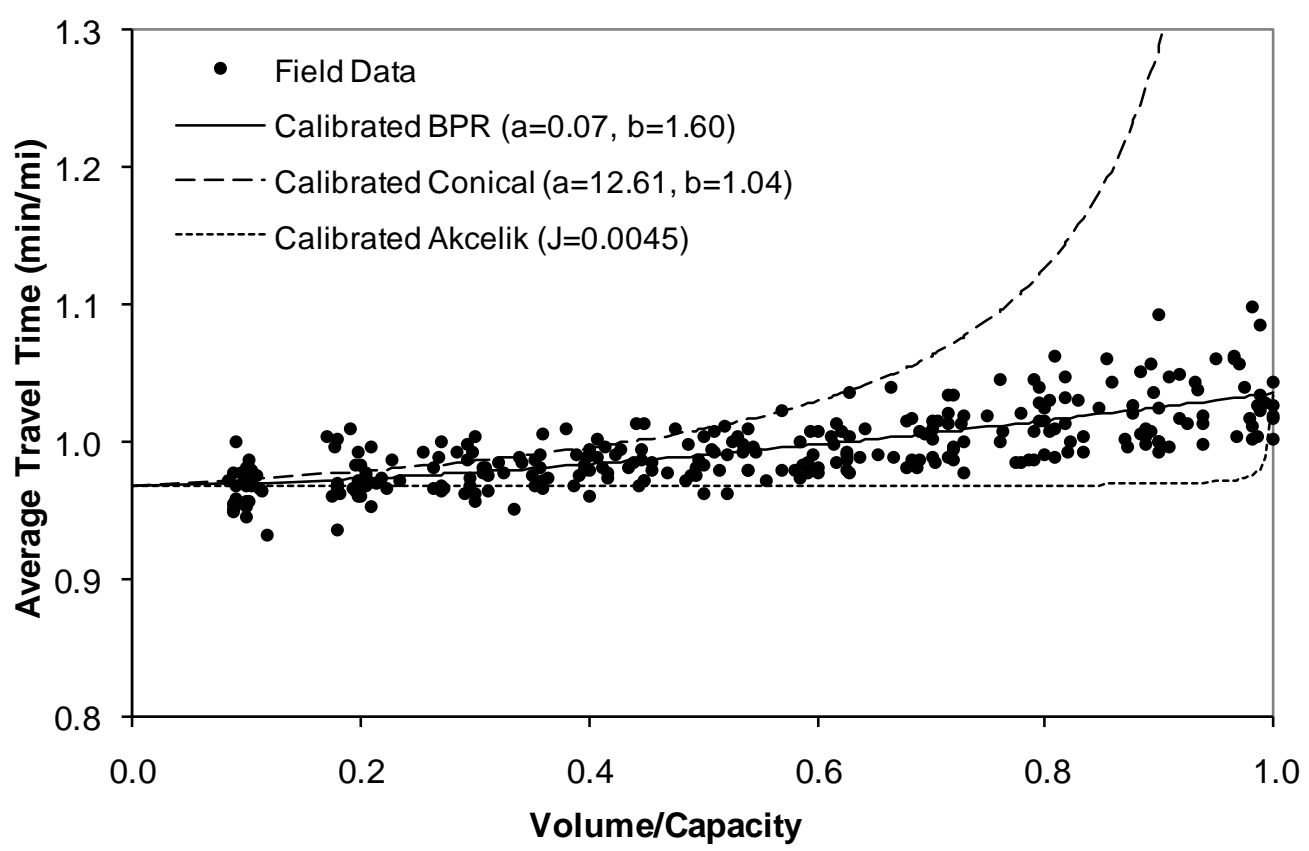

Figure 4.15. Comparison of calibrated travel time estimation functions against field data for $v / c<1$ for the left lane at milepost 1.92

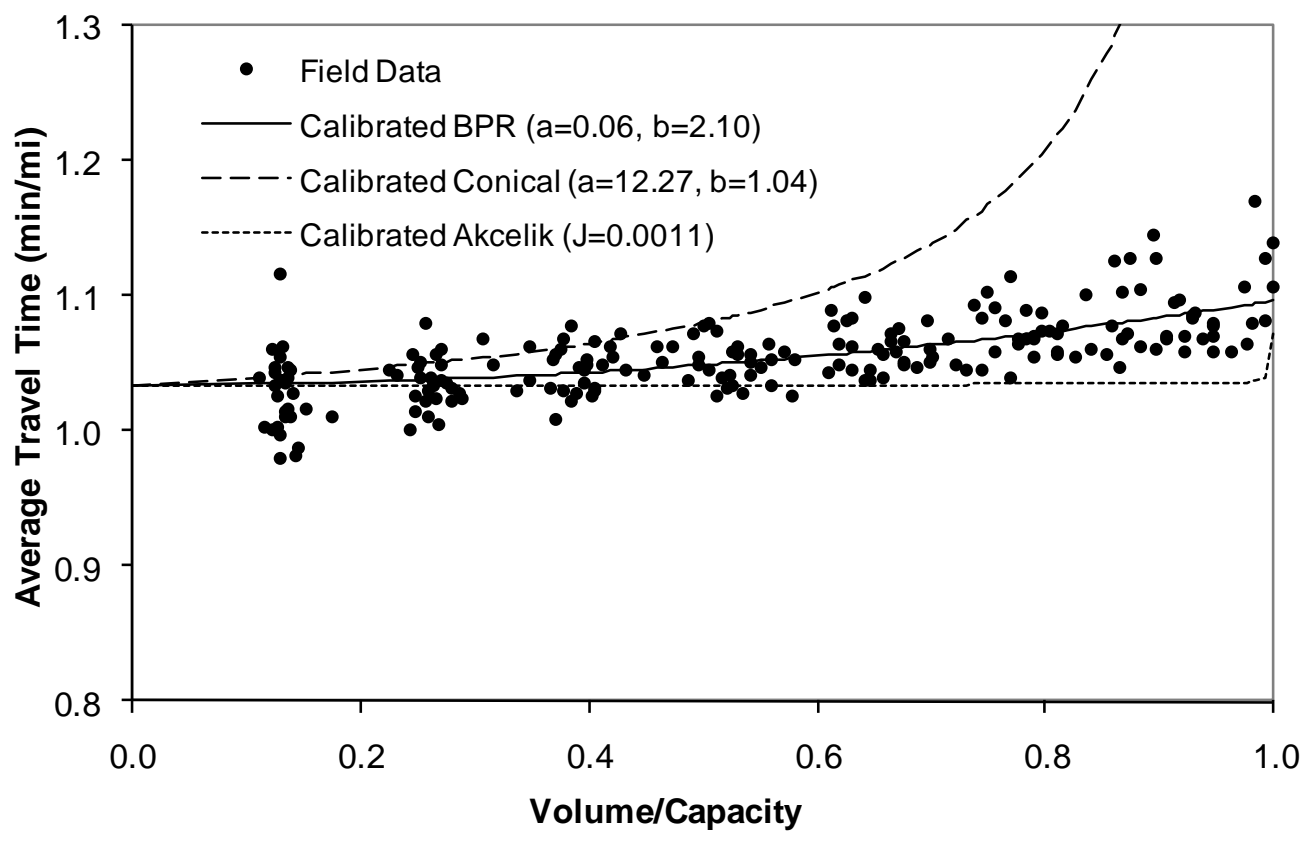

Figure 4.16. Comparison of calibrated travel time estimation functions against field data for $\mathrm{v} / \mathrm{c}<1$ for the right lane at milepost 1.92 


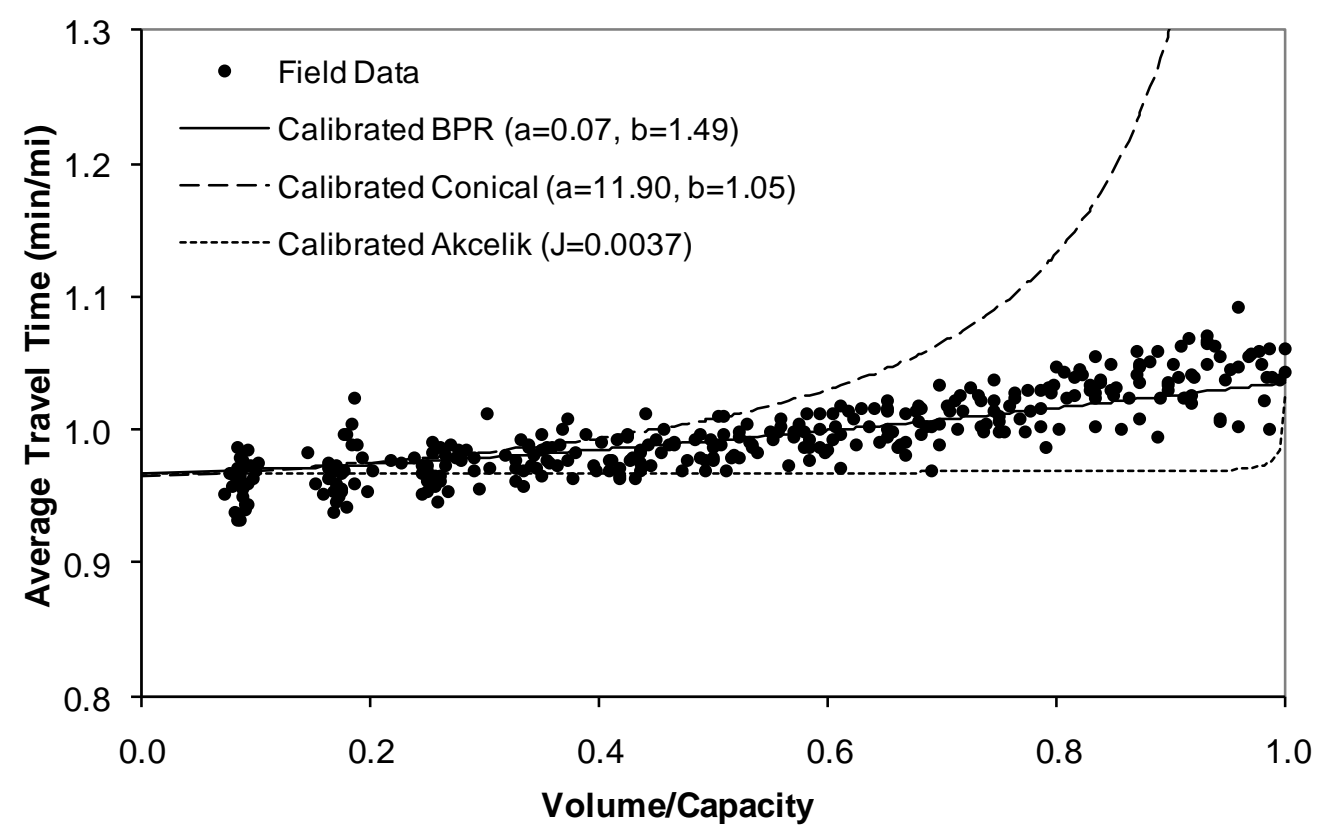

Figure 4.17. Comparison of calibrated travel time estimation functions against field data for $v / c<1$ for the left lane at milepost 3.12

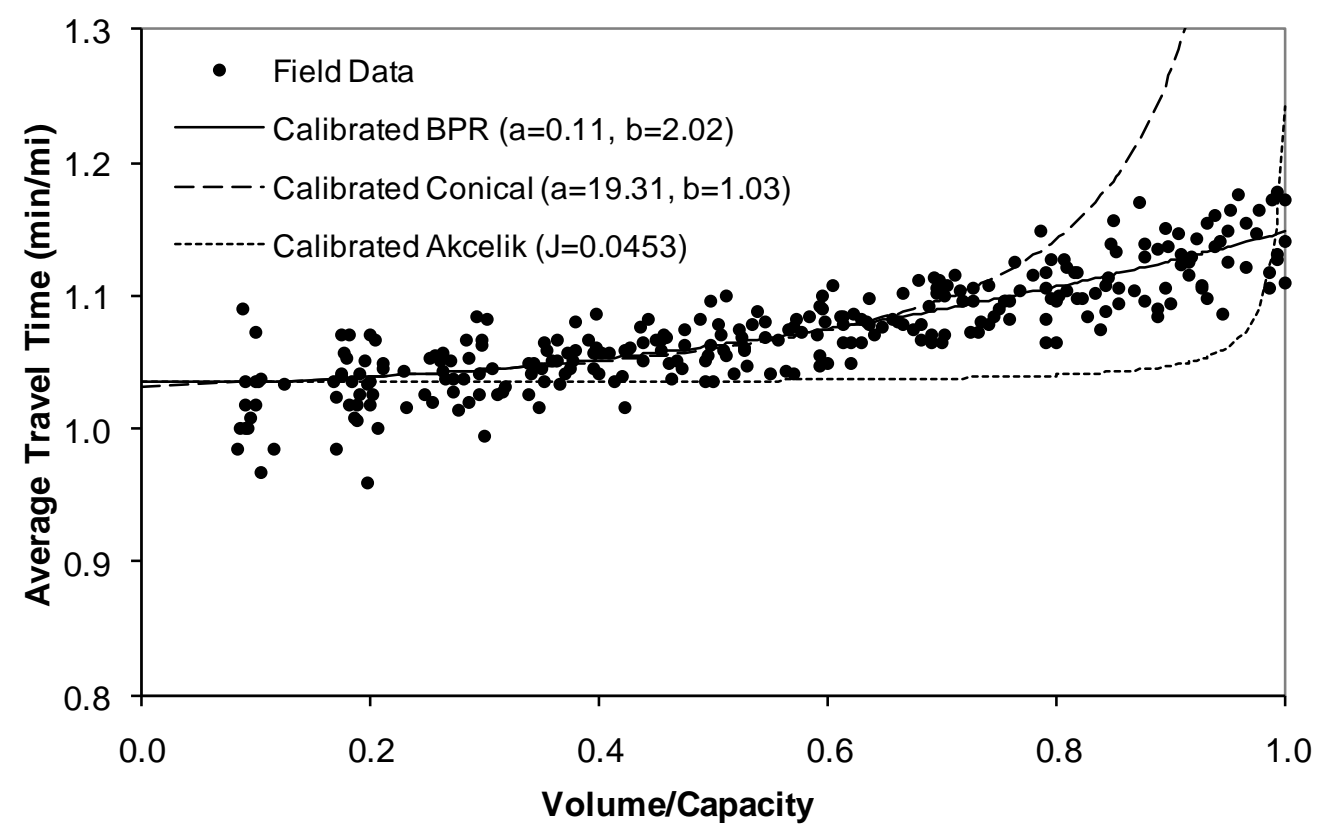

Figure 4.18. Comparison of calibrated travel time estimation functions against field data for $\mathrm{v} / \mathrm{c}<1$ for the right lane at milepost 3.12 
Figures 4.19 to 4.22 show the residual values for each calibrated model at each location and in each lane. As can be seen, the systematic error previously observed in the standard BPR function does not longer exist in the calibrated BPR function while the calibrated conical function and the calibrated Akçelik function still suffer from a systematic error. For the calibrated Akcelik function, the residuals are increasing positively except for v/c ratios close to 1 . For the calibrated conical function, the residuals increase negatively while the $\mathrm{v} / \mathrm{c}$ ratio gets larger. 

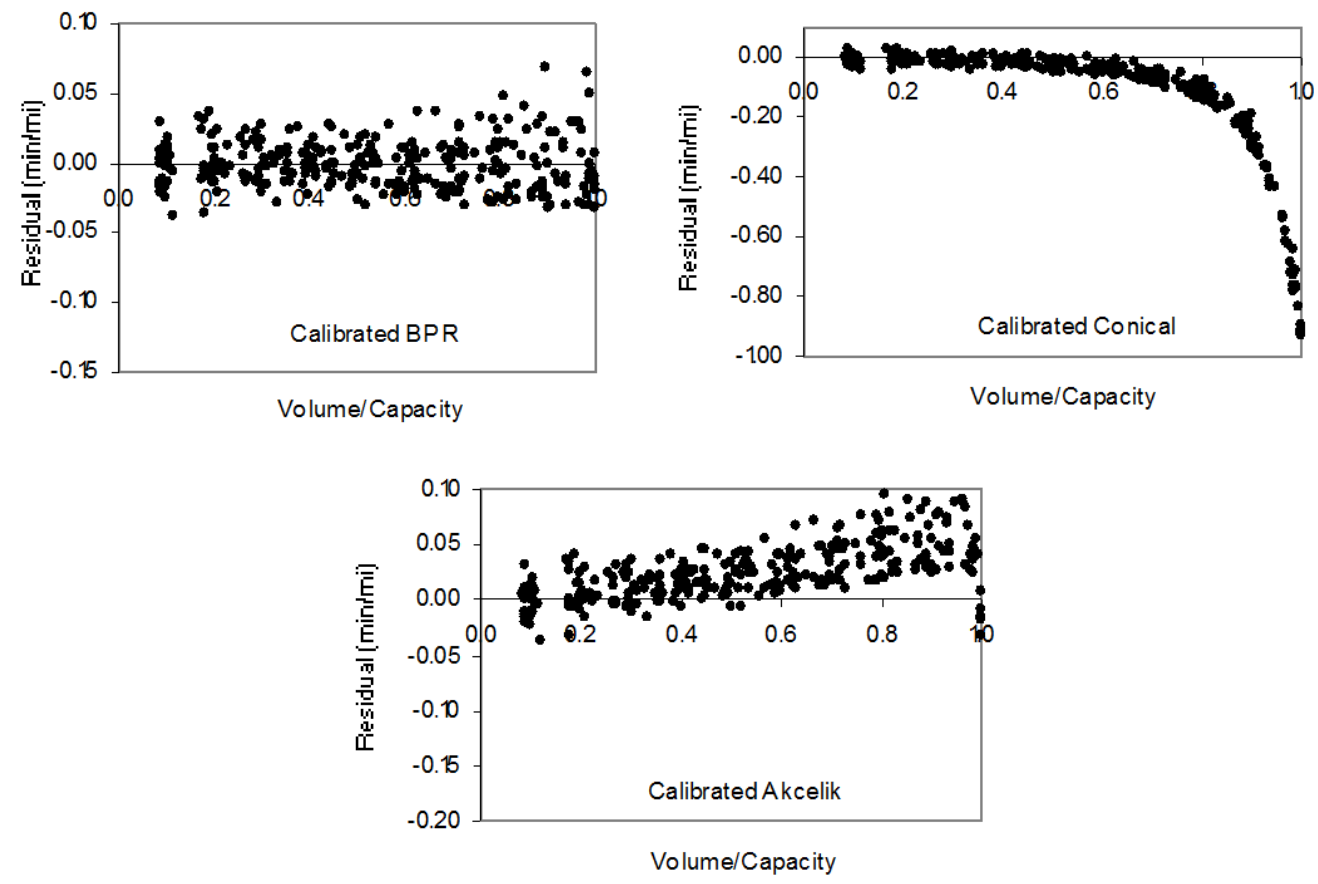

Figure 4.19. Residual values for the tested calibrated equations for the left lane at milepost 1.92
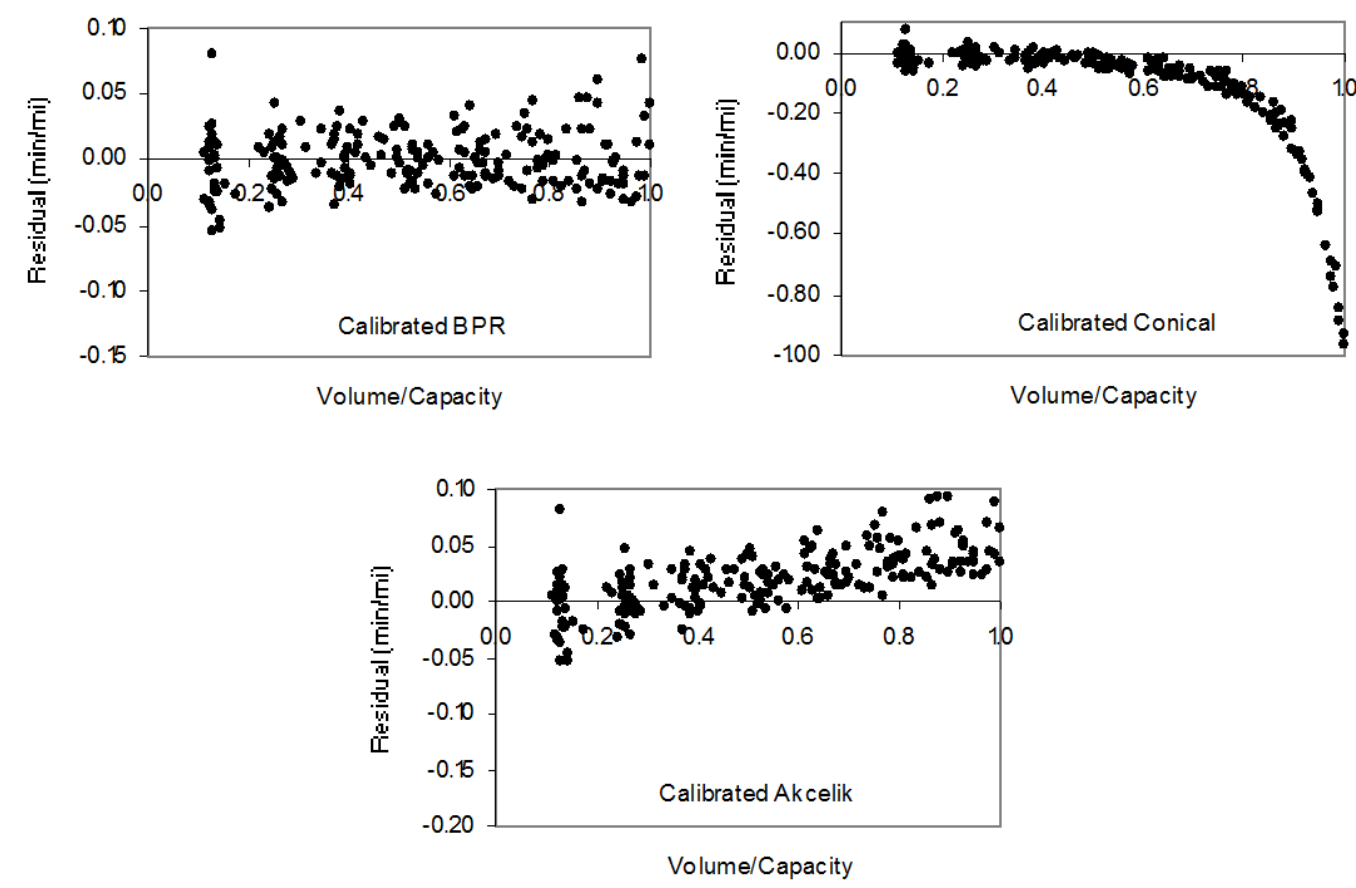

Figure 4.20. Residual values for the tested calibrated equations for the right lane at milepost 1.92 

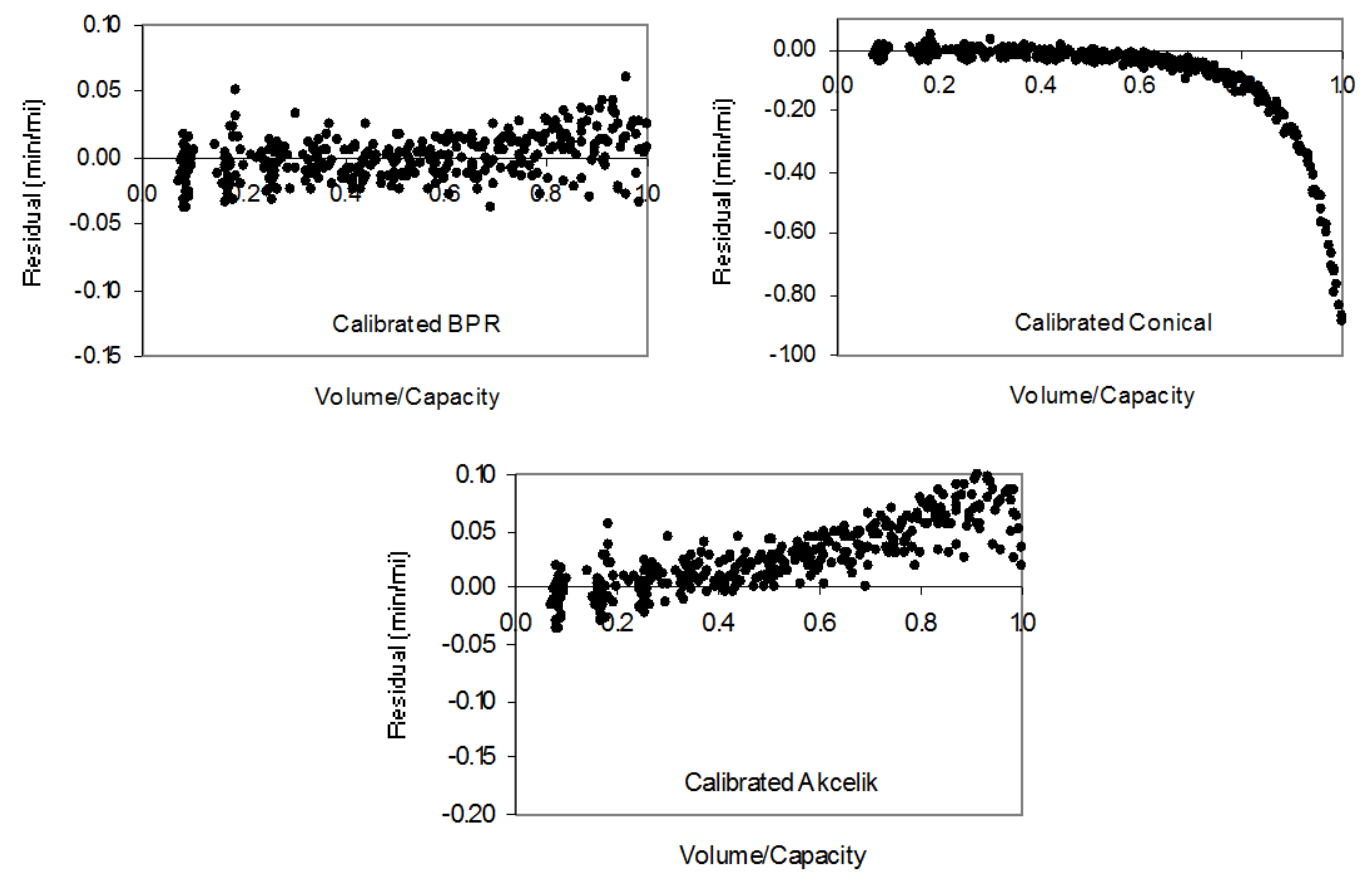

Figure 4.21. Residual values for the tested calibrated equations for the left lane at milepost 3.12
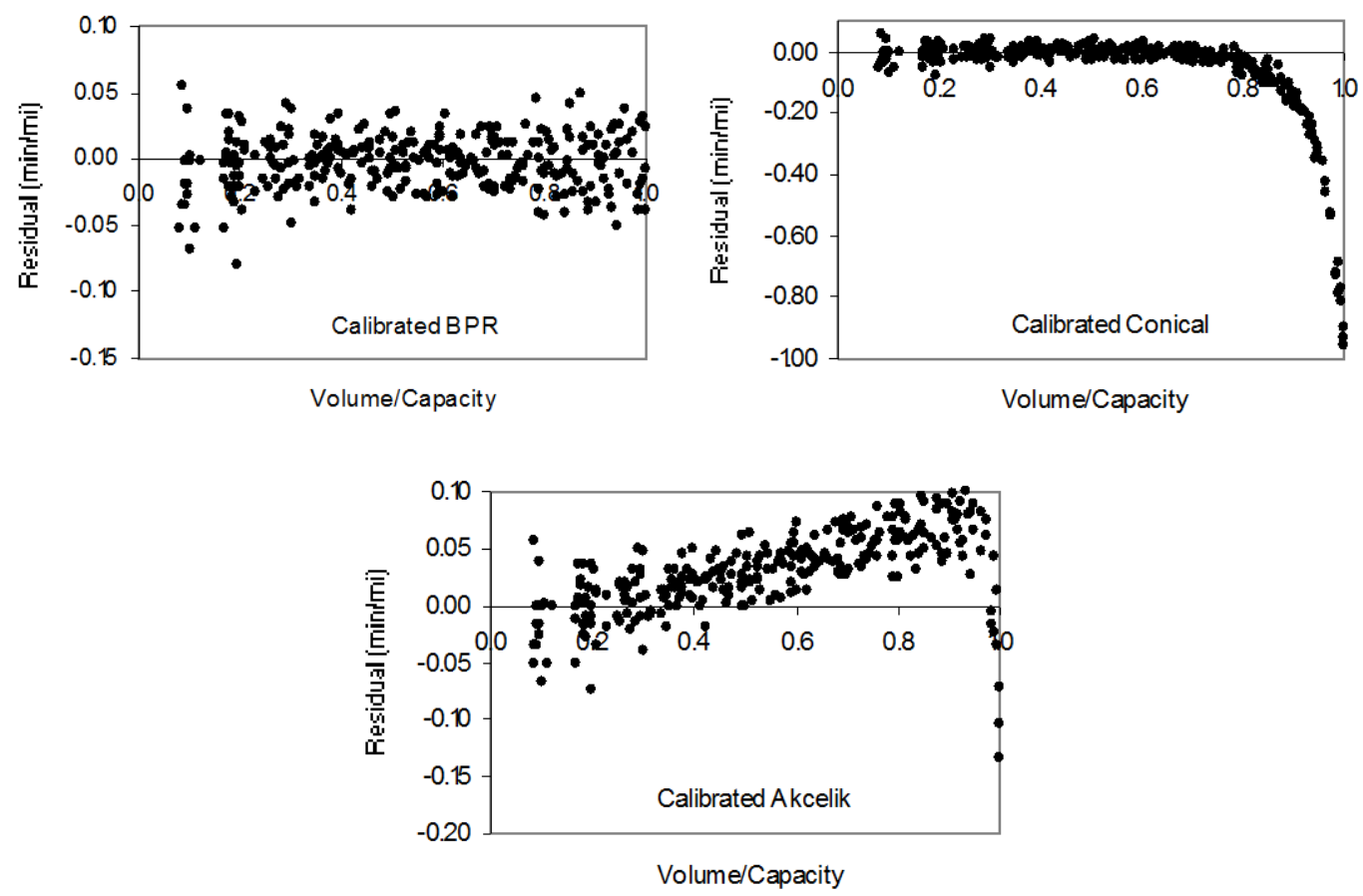

Figure 4.22. Residual values for the tested calibrated equations for the right lane at milepost 3.12 
For further exploration, the bias, RMSE, and MAE for each calibrated model are calculated and plotted for different v/c ratio ranges as described earlier (see figures 4.23 to 4.25 ). As can be seen, for the calibrated conical function and the calibrated Akçelik function, the values of RMSE and MAE are higher for $0.8<v / c \leq 1$ compared to other $v / c$ ratio ranges. For the calibrated BPR function, the RMSE and MAE remain roughly constant for all the $\mathrm{v} / \mathrm{c}$ ratio ranges. The bias for the calibrated BPR function is almost zero while other calibrated functions have higher values of bias.

Table 4.3 shows a comparison of the bias, RMSE, and MAE for uncalibrated and calibrated travel time estimation functions. A considerable improvement in bias, RMSE, and MAE is achieved by calibrating the tested functions. 

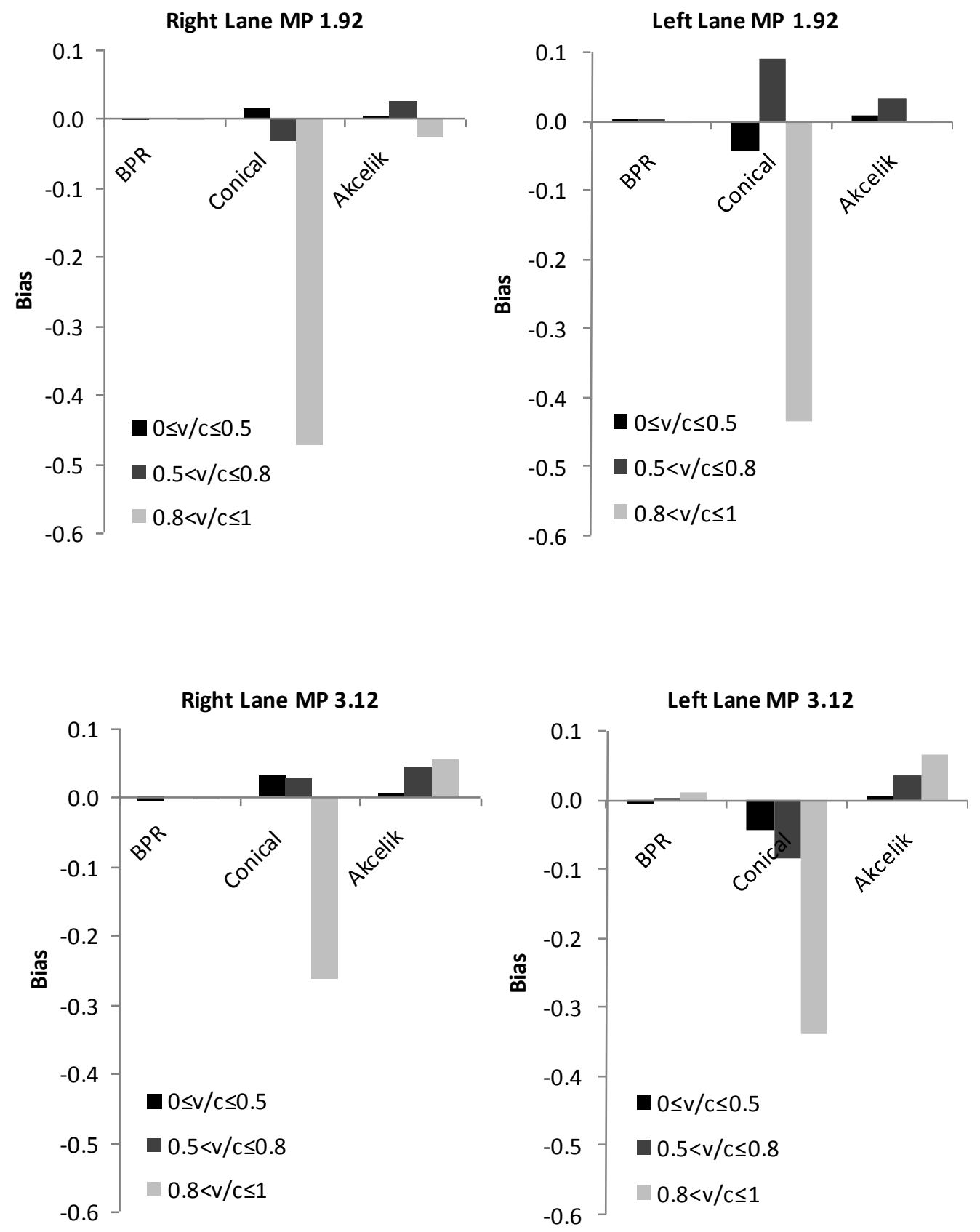

Figure 4.23. Bias values of calibrated models for different ranges of $v / c$ ratio 

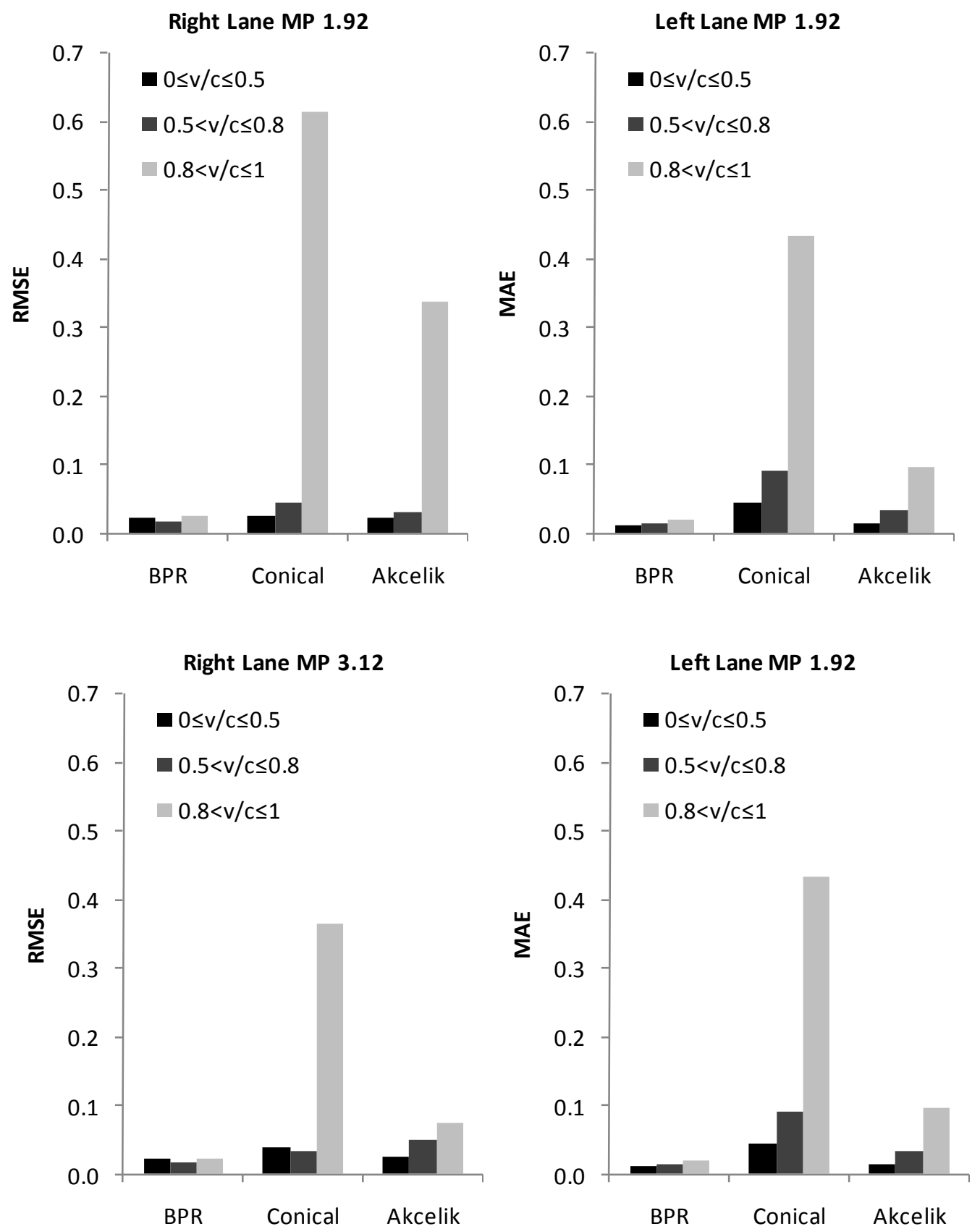

Figure 4.24. RMSE values of calibrated models for different ranges of v/c ratio 

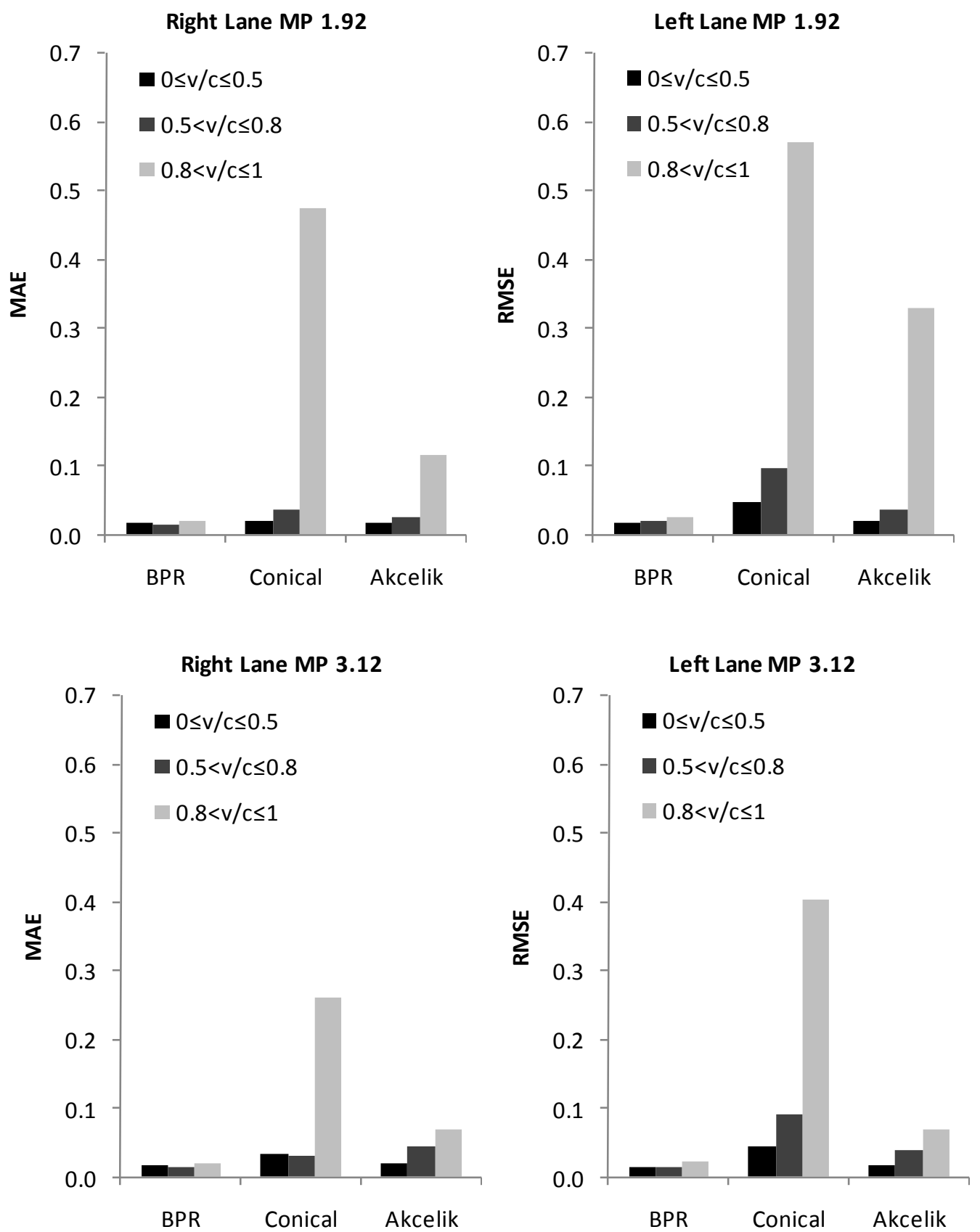

Figure 4.25. MAE values of calibrated models for different ranges of $v / c$ ratio 


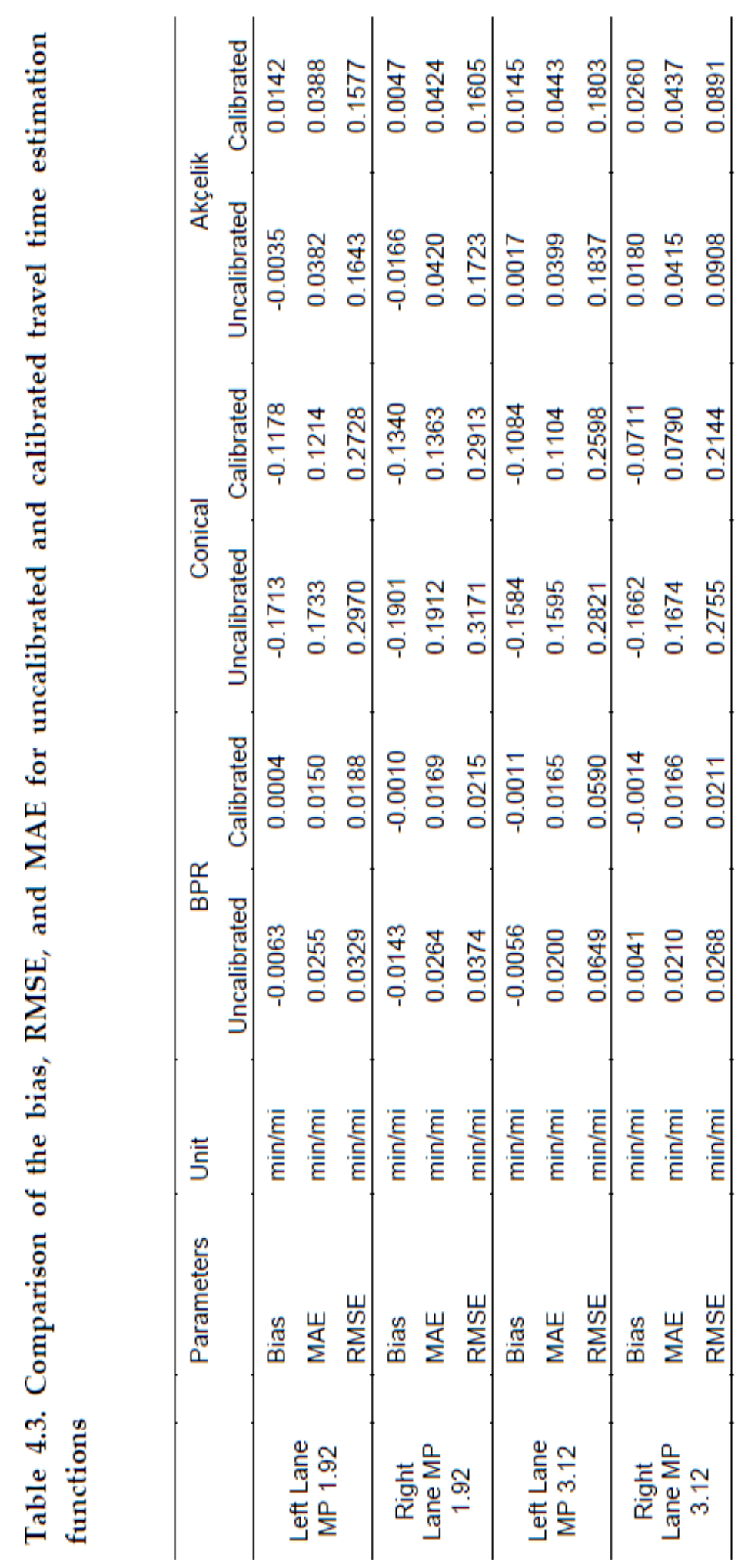




\subsection{Capacity as a Random Variable}

As mentioned earlier, literature suggests that the magnitude of the freeway capacity is not a single, unchanging numerical value. Figures 4.26 and 4.27 illustrate the empirical cumulative distribution of breakdown flows and maximum flows using 5-minute aggregated data for the selected 30 days. A statistically significant difference at $99 \%$ level based on chi-square test results between capacity distributions at each lane is observed. This implies the possible effects of lane and road characteristics on capacity. At milepost 1.92, a 0.25 mile weaving segment is located upstream of the Beaverton-Hillsdale Hwy and at milepost 3.12, a 0.32 mile weaving segment is located upstream of the Denney Rd on-ramp. The left lane at milepost 3.12 has the largest range of capacities while the right lane at milepost 1.92 has the lowest range of capacities. At both locations, the left lanes experienced higher values of capacities. 


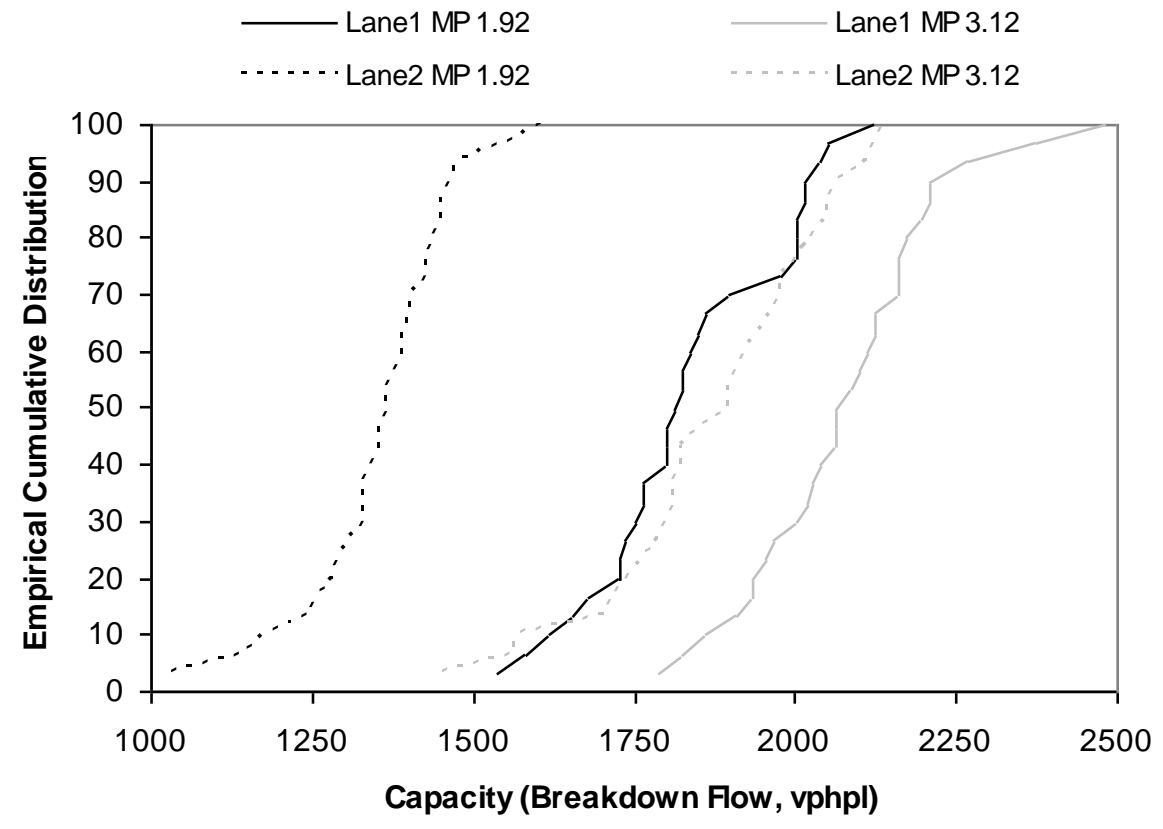

Figure 4.26. Empirical cumulative distribution of 5-minute breakdown flows

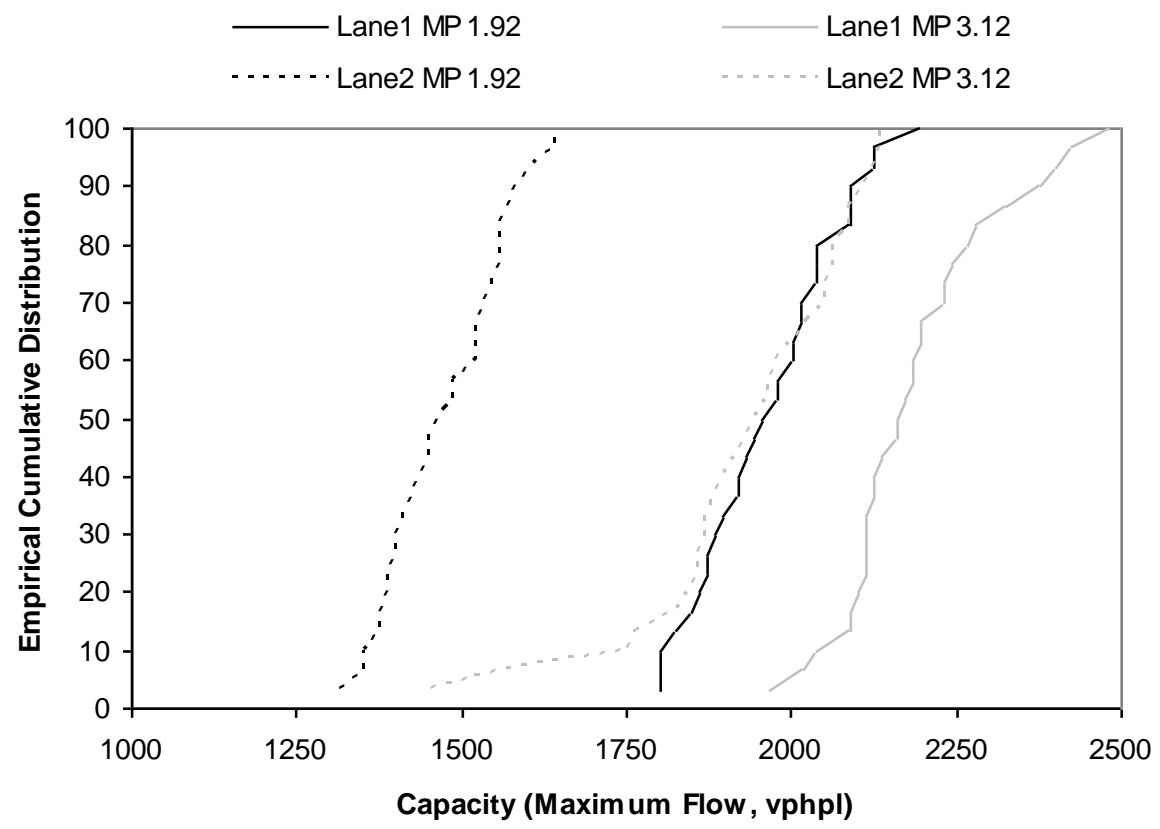

Figure 4.27. Empirical cumulative distribution of 5-minute maximum flows 
The difference observed between capacity distributions at milepost 1.92 and milepost 3.12 could be because of two possible reasons: 1) longer weaving segment upstream of the milepost 3.12 compared to the milepost 1.92 and 2) higher ramp flows at milepost 1.92 compared to milepost 3.12. Figure 4.28 shows the average flow of the on-ramp at milepost 1.92 and milepost 3.12. As can be seen, the average flow of the on-ramp at milepost 1.92 is larger than the average flow of the on-ramp at milepost 3.12 throughout the study time period. As mentioned earlier, this could be one of the reasons for lower capacity of the right lane at milepost 1.92 compared to the right lane at milepost 3.12.

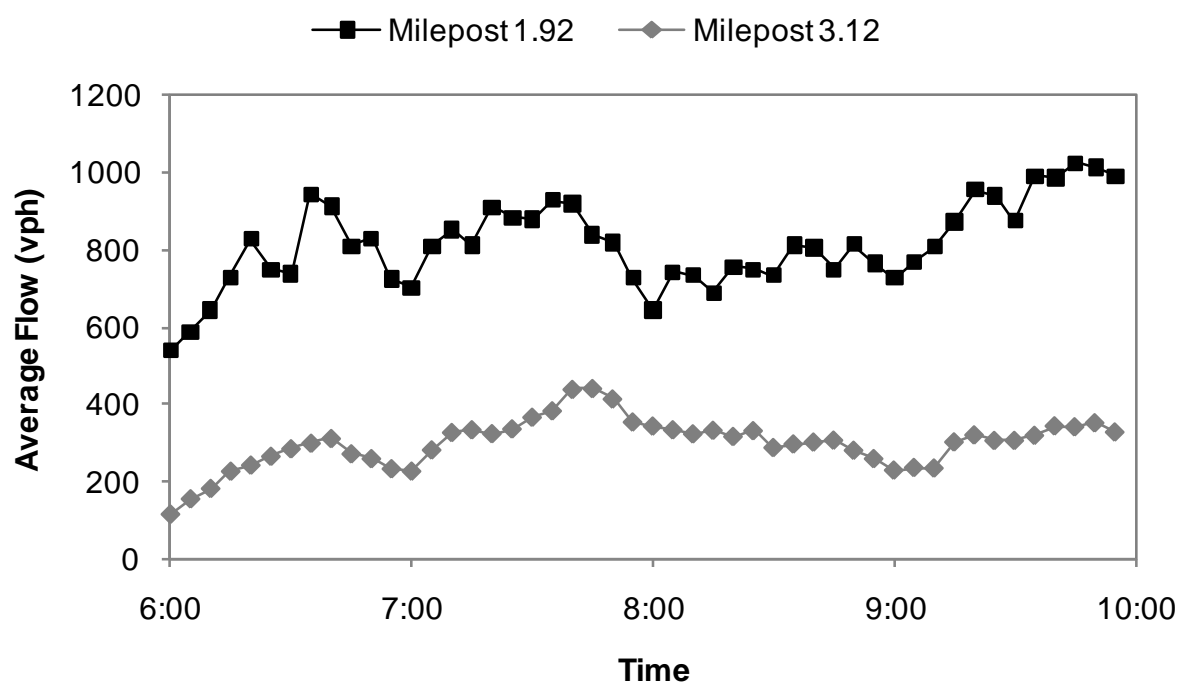

Figure 4.28. Average on-ramp volumes at study locations 
The 15-minute interval, which is typically used in capacity analyses,

is also selected for analysis because it provides an interesting comparison to the 5-minute interval. Figures 4.29 and 4.30 illustrate the empirical cumulative distribution of 15-minute breakdown flows and maximum flows for the selected 30 days. It is apparent from the figures that if the aggregation level is increased to 15 minute, the shape of the cumulative distribution does not differ considerably. As expected, the 15-minute cumulative distributions have shorter end tails compared to the 5-minute cumulative distributions. Table 4.4 shows the median values of measured breakdown flows and maximum flows using 5- and 15-minute aggregated data. The median value of measured 5minute breakdown flow is $1 \%-5 \%$ larger than the median value of measured 15-minute breakdown flow. The median value of measured 5-minute maximum flow is 5\%-9\% larger than the median value of measured 15 -minute maximum flow.

Table 4.4. Median measured breakdown flows and maximum flows

\begin{tabular}{lcccc}
\hline & \multicolumn{2}{c}{ Breakdown Flow (vphpl) } & \multicolumn{2}{c}{ Maximum Flow (vphpl) } \\
\cline { 2 - 5 } & $5 \mathrm{~min}$ & $15 \mathrm{~min}$ & $5 \mathrm{~min}$ & $15 \mathrm{~min}$ \\
\hline Left Lane, MP 1.92 & 1,818 & 1,782 & 1,968 & 1,794 \\
Right Lane, MP 1.92 & 1,368 & 1,346 & 1,476 & 1,358 \\
Left Lane, MP 3.12 & 2,076 & 2,048 & 2,166 & 2,054 \\
Right Lane, MP 3.12 & 1,896 & 1,806 & 1,956 & 1,806 \\
\hline
\end{tabular}


The results confirm literature that the magnitude of the freeway capacity is not a single, unchanging numerical value. Flow breakdown and maximum flow can occur over a wide range of flow rates. 


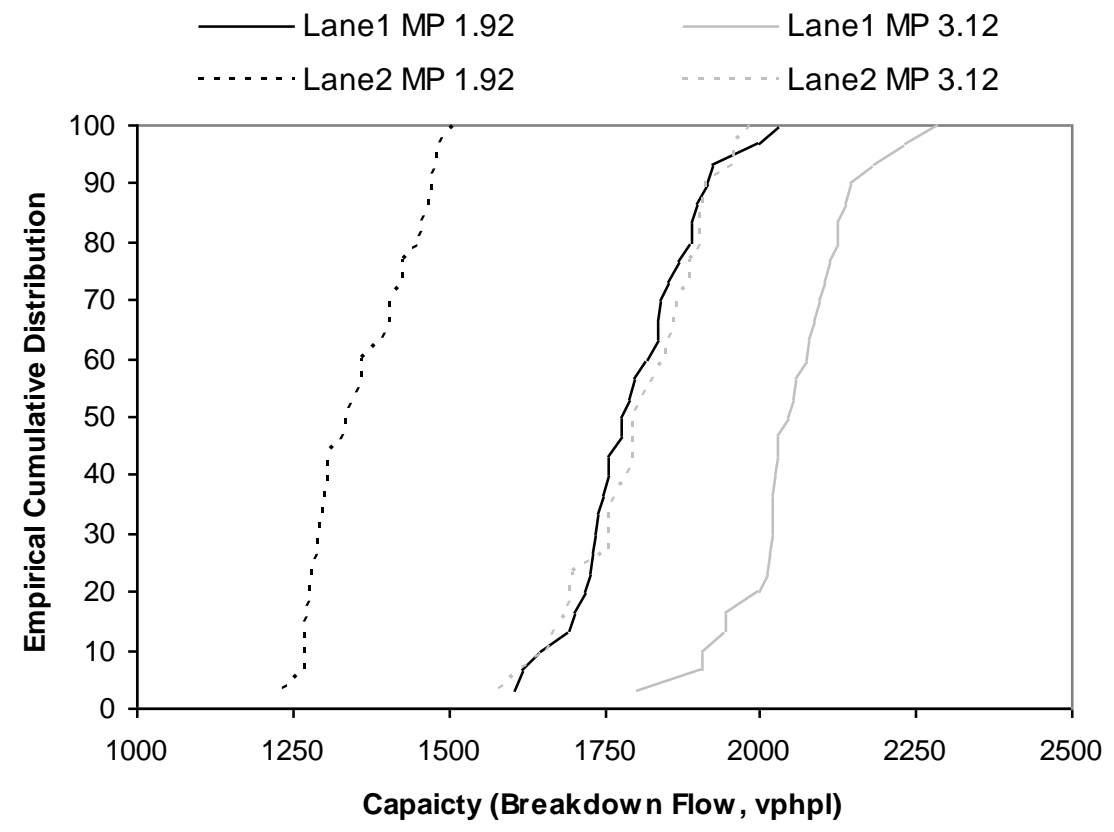

Figure 4.29. Empirical cumulative distribution of 15-minute breakdown flows

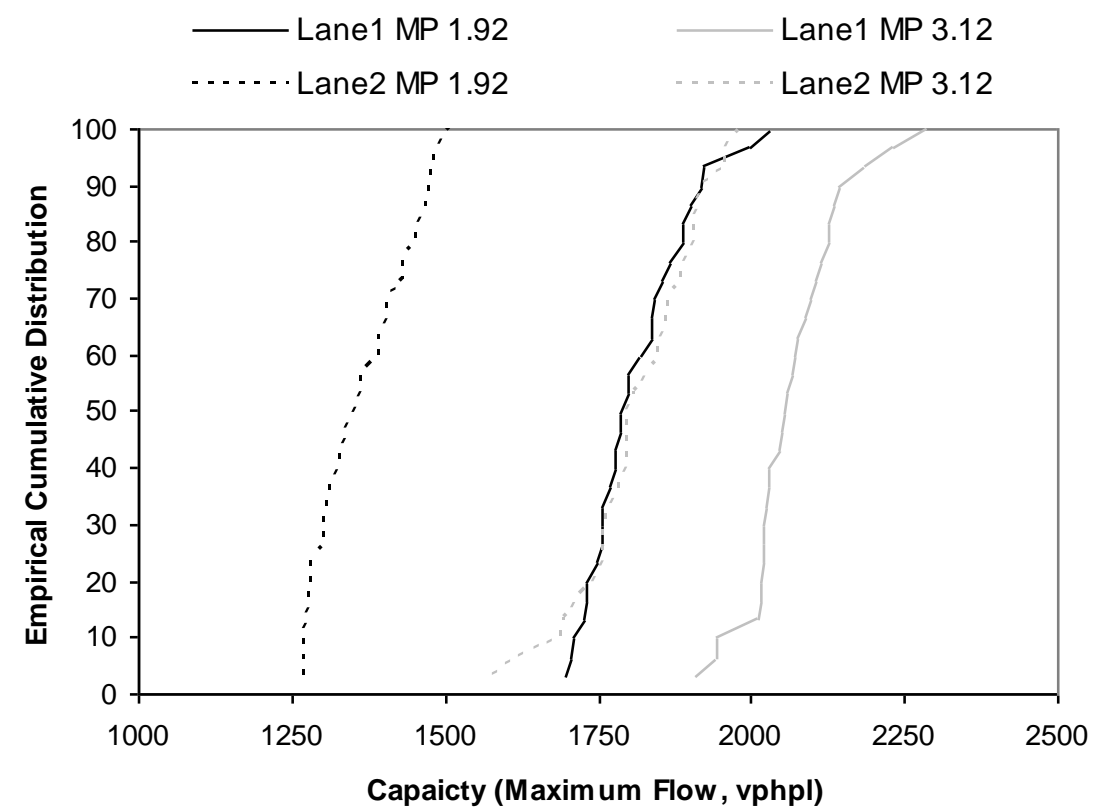

Figure 4.30. Empirical cumulative distribution of 15-minute maximum flows 


\subsection{Summary}

The analysis described in this chapter resulted in the following key conclusions:

- Results in section 4.1 show that lanes do not necessarily have similar characteristics. It is shown that free-flow speed, speed at capacity and capacity have different values in different lanes. Therefore, the approach of using relationships between speed and flow on individual lanes is beneficial because it makes it possible to model freeway segments at which the capacity for different reasons corresponds to fraction of lanes.

- The accuracy of the existing speed-flow models with default parameters are explored in section 4.2. The standard BPR and METRO updated functions overestimate travel times for v/c ratios close to 1 . The conical function highly overestimates travel times for $\mathrm{v} / \mathrm{c}<1$. The conical function assumes that the travel time at capacity is two times larger than the free-flow travel time which is not always true. The Akçelik and HCM 2000 models underestimate 
travel times for $\mathrm{v} / \mathrm{c}<0.9$ and overestimate travel times for $\mathrm{v} / \mathrm{c}$ close to 1.

- The results presented in section 4.3 show that for $\mathrm{v} / \mathrm{c}<1$, the BPR function has the best overall performance among models tested when calibrated.

- The results of the analysis described in section 4.4 suggest that capacity is not a deterministic measure. The results show that it is worthwhile to apply an alternative definition of capacity in traffic assignment in light of the probabilistic nature observed.

Similar analyses can be done using pooled data over the lanes to investigate the speed-flow relationships at link level and to find out the possible advantages of using lane specific speed-flow relationships. 


\subsection{MODIFICATIONS}

In this chapter some deterministic modifications, based on calibration results and queuing theory, will be proposed for the speed-flow relationship for both static and dynamic traffic assignment applications. A probabilistic modification will also be presented in order to make the proposed formulation capture variations in capacity. The probabilistic modification can be applied to any $\mathrm{v} / \mathrm{c}$ ratio based speed-flow model.

\subsection{Deterministic Modification}

Shortcomings of different speed-flow models have been discussed in previous chapters. Calibration results showed that the BPR function has the best overall performance for $\mathrm{v} / \mathrm{c}<1$, when calibrated. For $\mathrm{v} / \mathrm{c}>1$, it was shown that the Akçelik model and the HCM 2000 model, which is a modified version of the Akçelik model, have the highest consistency with queuing theory among tested models. However, estimated travel time by the Akçelik model for congested conditions is based on the average delay in the queue. As discussed in chapter 2, the average delay $\left(D_{A}\right)$ for stationary arrival and departure rates for oversaturated conditions can be expressed as: 


$$
D_{A}=\frac{1}{2} T(X-1)
$$

where $X$ is the $v / c$ ratio and $T$ is the time period which the demand rate persists.

The actual delay experienced by individual vehicles in a queue depends on the vehicle's departure time or the time when the vehicle joins the queue. Figure 5.1 illustrates a graphical representation of the delay experienced by vehicle $i$ in a queue with stationary arrival and departure rates where $t_{i}$ is the time when the vehicle joins the queue, $d_{i}$ is the delay experienced by the vehicle $i$, and $T$ is the time interval during which an average demand flow rate persists.

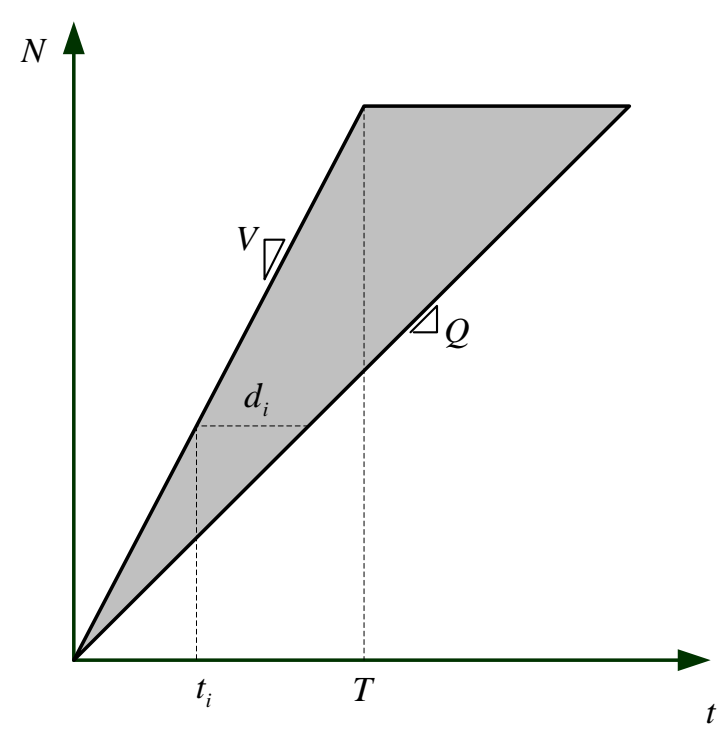

Figure 5.1. The delay experienced by vehicle $i$ in a queue with stationary arrival and departure rates 
Note that different graphical representations of delay in a queue can be plotted and therefore different modifications can be proposed. The delay experienced by vehicle $i$, as shown in figure 5.1., is calculated as follows:

$$
d_{i}=t_{i}\left(\frac{V}{Q}-1\right), 0 \leq t_{i} \leq T \text { and } i=1 \text { to } n
$$

Assuming the queue forms directly upstream of the bottleneck and takes considerable physical space, the actual delay experienced by vehicle $i$ is calculated as follows as explained in section 2.4:

$$
\begin{gathered}
d_{i}=\phi \times t_{i}\left(\frac{V}{Q}-1\right) \\
\phi=\frac{1}{\left(1-\frac{v_{q}}{v_{f}}\right)}
\end{gathered}
$$

where $v_{q}$ is the average speed of vehicles within the queue and $v_{f}$ is the average speed of vehicles approaching the queue (free-flow speed). The speed $v_{q}$ can be estimated using a rescaled cumulative curve. For example, in the rescaled cumulative curve of speed between 6:00 to 10:00 at milepost 4.35 on January 22, 2010 which was shown in figure 3.5, the interpolated lines with constant slope represent stationary traffic conditions. Between the bottleneck activation and deactivation times, the slope of the interpolated line represents 
the average speed within the queue which is $22 \mathrm{mph}$ in this example. Figure 5.2 shows the empirical cumulative distribution of vehicle's speed within the queue at the study locations. As can be seen, higher speeds within the queue are observed on the left lanes compared to the right lanes at both locations.

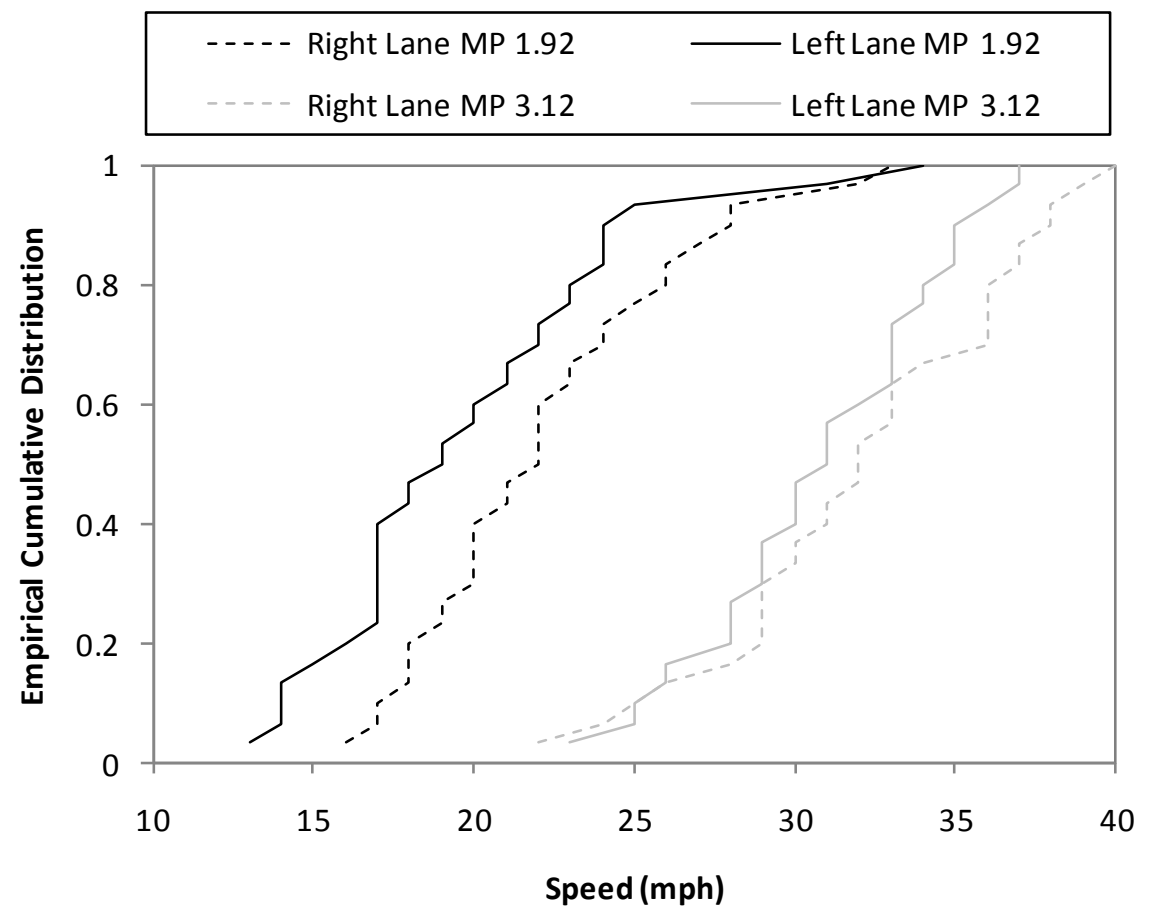

Figure 5.2. Empirical cumulative distribution of vehicles' speed within the queue. 
Travel time

(min/mile)

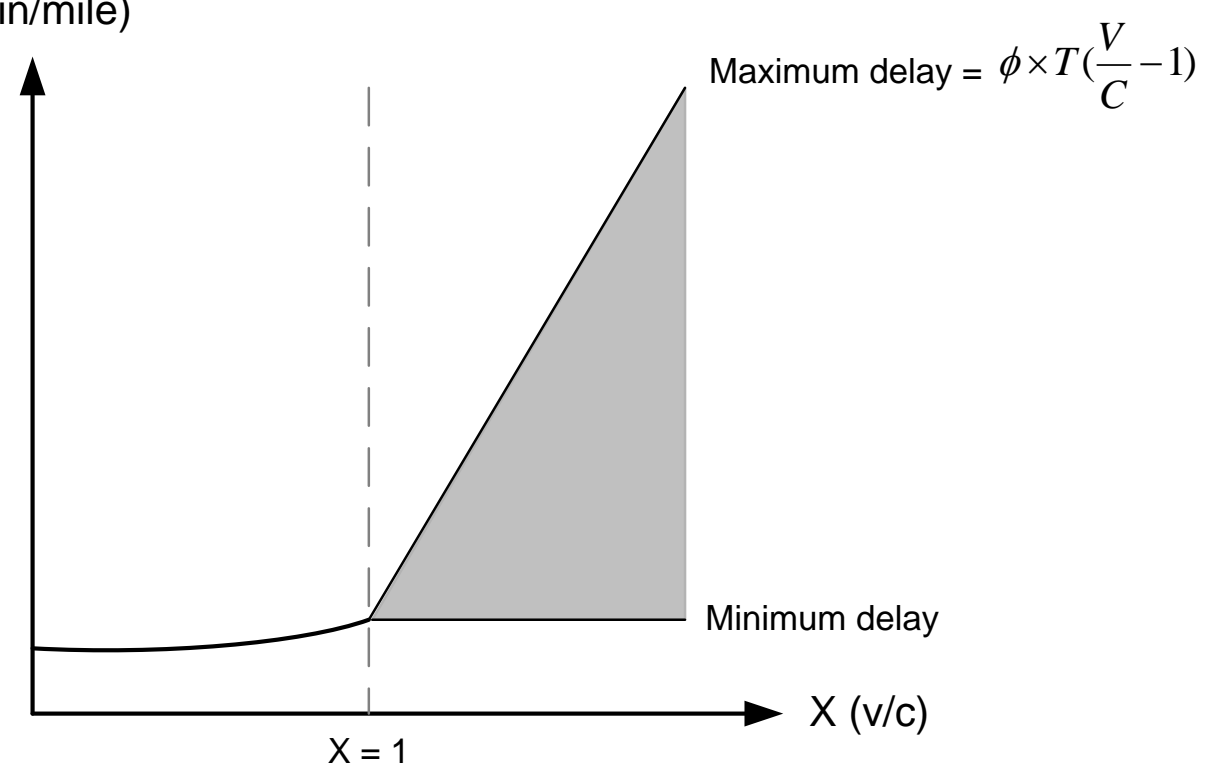

Figure 5.3. Time dependent travel time and v/c ratio relationship

Figure 5.3 illustrates the time dependent relationship between travel time and v/c ratio considering vehicle's arrival time at the queue. The first vehicle $\left(i=1, t_{i}=0\right)$ that joins the queue experiences a minimum delay of close to zero and the last vehicle $\left(i=\mathrm{n}, t_{i}=T\right)$ that joins the queue experiences a maximum delay equal to $\phi \times T\left(\frac{V}{C}-1\right)$ where $T$ is bounded by zero and bottleneck duration time as follows:

$$
0 \leq T<\text { bottleneck duration time }
$$


where the bottleneck duration time at each lane is defined as follows:

bottleneck duration time $=$ bottleneck deactivation time - bottleneck

activation time

\subsubsection{Speed-Flow Relationship at Link Level}

The analysis reported in chapter 4 concentrated on speed-flow relationships on individual lanes; similar analyses can be performed at the link level by simply pooling lane data. A previous study by Hurdle et al. (1997) showed that the speed-flow curve for a full roadway is significantly different from the curves for the individual lanes. Since traffic flow characteristics are not the same in different lanes as shown earlier, measuring free-flow speed, speed at capacity, and estimating total capacity of freeway over lanes may require a different approach that needs to be studied further. If $T$, as shown in figure 5.1 and defined in equation 5.5 , is being calculated over all the lanes to be used in speed-flow relationship at link level, bottleneck deactivation and activation times can be estimated as follows:

- bottleneck deactivation time $=\min \{$ bottleneck deactivation

time at lane 1 , bottleneck deactivation time at lane 2$\}$ 
- bottleneck activation time $=\max \{$ bottleneck activation time at

lane 1 , bottleneck activation time at lane 2$\}$

Figure 5.4 shows the empirical cumulative distributions of the bottleneck activation and deactivation times over all the lanes at milepost 1.92 and milepost 3.12 .

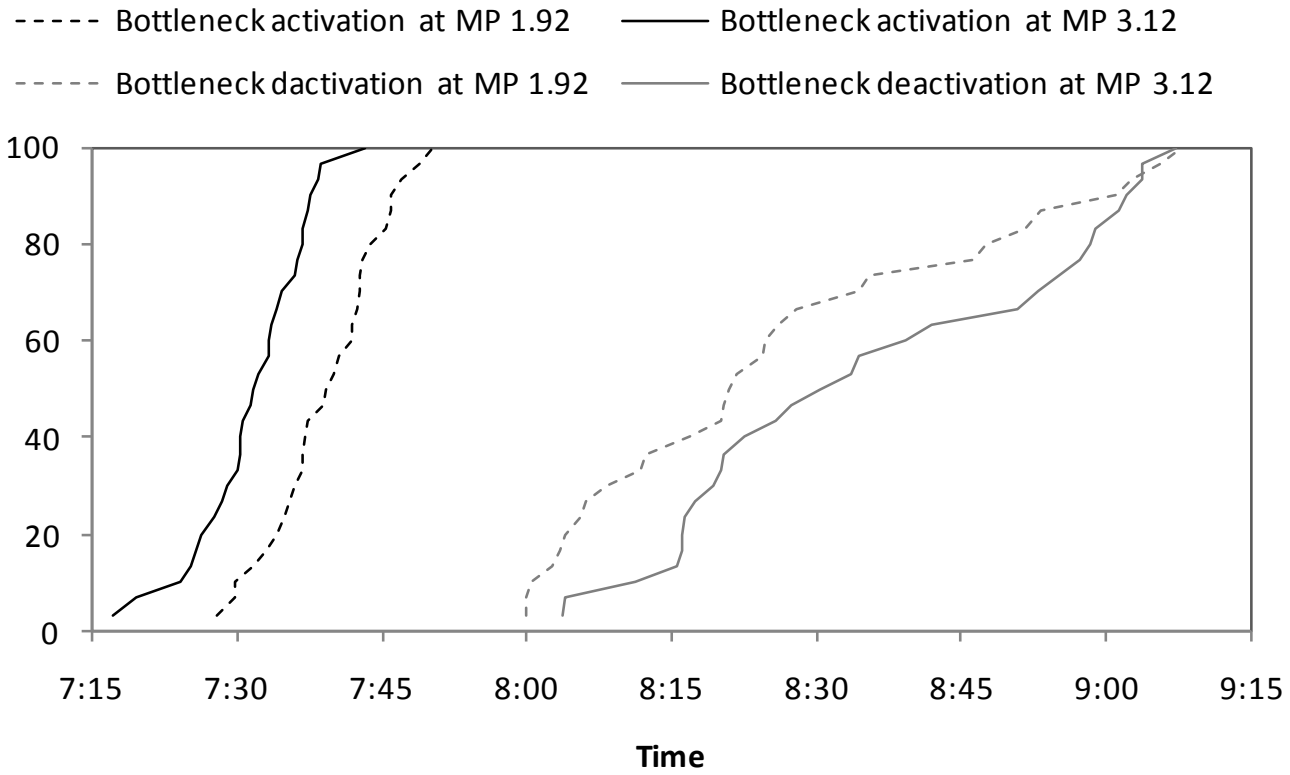

Figure 5.4. Empirical cumulative distributions of bottleneck activation and deactivation times over all the lanes at milepost 1.92 and milepost 3.12

Table 5.1 provides key statistical descriptors for the free-flow speed, speed at capacity, speed within the queue, breakdown flow, and maximum flow over the lanes at each location. Comparing these values with previously 
stated values of measured traffic parameters at each lane indicates that the traffic parameters may vary considerably among lanes and over the lanes.

Table 5.1. Statistical description of free-flow speed, speed at capacity, speed within the queue, breakdown flow, and maximum flow at link level

\begin{tabular}{lccccc}
\hline & min & mean & median & max & stdv \\
\cline { 2 - 6 } & \multicolumn{5}{c}{ Milepost 1.92 } \\
Free flow speed (mph) & 58 & 60 & 60 & 61 & 1 \\
Speed at capacity (mph) & 46 & 53 & 53 & 57 & 3 \\
Speed within the queue (mph) & 15 & 21 & 21 & 34 & 4 \\
Breakdown flow (vph) & 2,940 & 3,234 & 3,222 & 3,732 & 201 \\
Maximum flow (vph) & 3,192 & 3,429 & 3,444 & 3,732 & 164 \\
& \multicolumn{5}{c}{ Milepost 3.12 } \\
Free flow speed (mph) & 58 & 60 & 60 & 63 & 1 \\
Speed at capacity (mph) & 48 & 52 & 52 & 55 & 2 \\
Speed within the queue (mph) & 23 & 32 & 32 & 39 & 4 \\
Breakdown flow (vph) & 3,420 & 4,008 & 3,990 & 4,596 & 271 \\
Maximum flow (vph) & 3,720 & 4,151 & 4,140 & 4,596 & 191 \\
\hline
\end{tabular}

Figure 5.5 shows the calibrated BPR curves (for $\mathrm{v} / \mathrm{c}<1$ ) separately for the left lane, right lane, and over the lanes at each study location against pooled field data. As can be seen, at both locations, the calibrated BPR function for the left lane estimates higher travel times than the calibrated BPR 
function over the lanes while the calibrated BPR function for the right lane estimates lower travel times than the calibrated BPR function over the lanes.
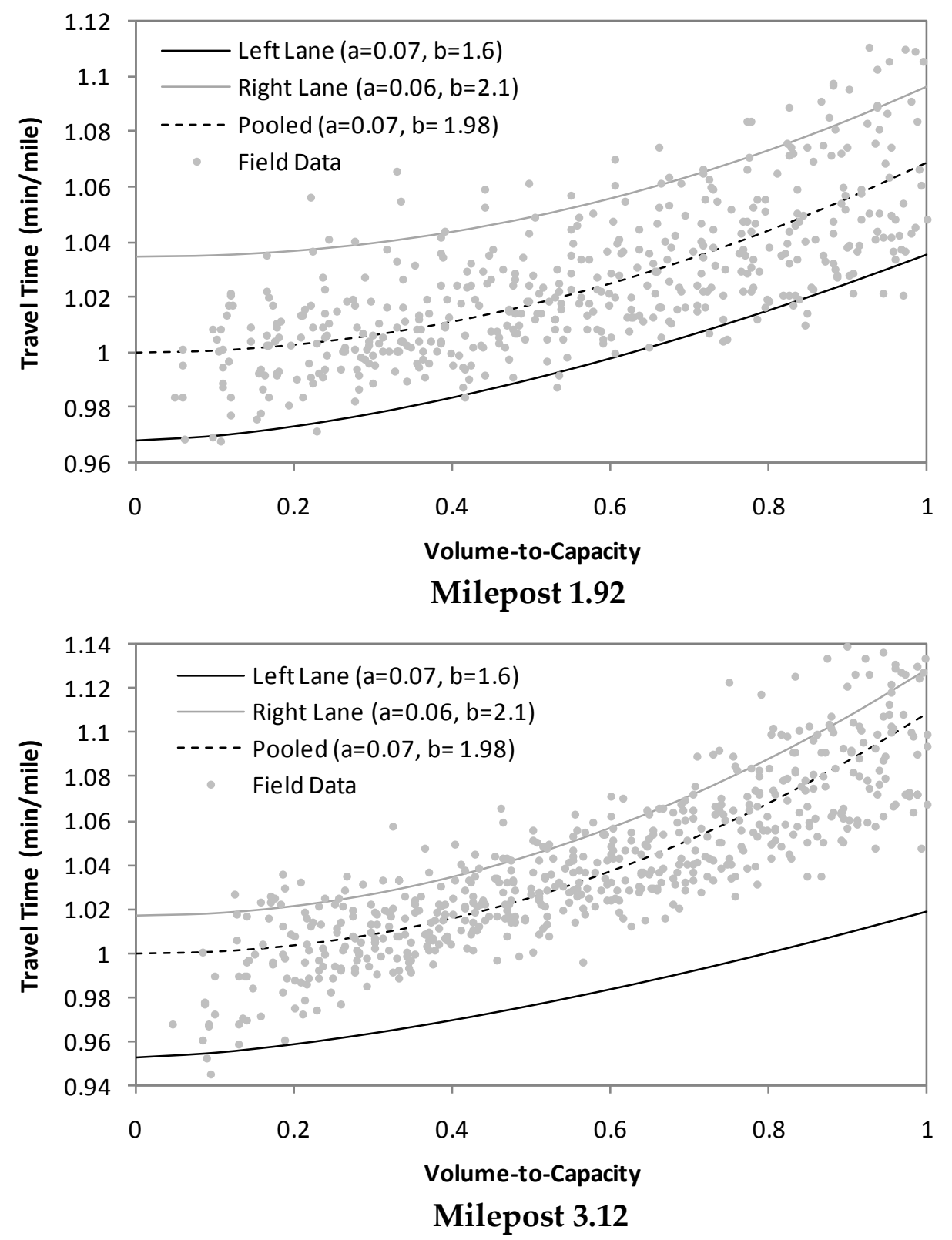

Figure 5.5. Comparison of the calibrated BPR functions for $v / c<1$ at lane and link levels 
Toward this end, the following speed-flow formulation is proposed.

Equation 5.3 can be used for v/c $>1$ to account for the vehicle's arrival time at the qeueu. For v/c $<1$, the calibrated BPR function is suggested:

$$
t=\left\{\begin{array}{cl}
t_{0}\left(1+a\left(\frac{v}{c}\right)^{b}\right) & \frac{v}{c} \leq 1 \\
t_{c}+\phi \times t_{i}\left(\frac{v}{c}-1\right) & \frac{v}{c} \geq 1
\end{array}\right.
$$

where:

$t$ = average travel time per unit distance,

$t_{0}=$ free-flow travel time per unit distance,

$t_{c}=$ travel time at capacity per unit distance,

$t_{i}=$ time when the vehicle $i$ joins the queue,

$\frac{v}{c}=$ volume-to-capacity ratio,

$a$ and $b$ are calibrated BPR parameters, and $\phi$ is as defined in equation 5.4. For a congested corridor consisted of two or more links, $t_{i}$ for each link can be calculated using shockwave speed and link length. For example, assume a 2 mile corridor, connecting A to B, including two 1-mile long links. A bottleneck which is located at the end of the downstream link is activated at 8:00 A.M. and a queue backs up with a constant shockwave speed of $3 \mathrm{mph}$. A vehicle 
joins the queue at 8:30 A.M. Therefore, $t_{i}$ for the whole corridor is 30 minutes. Since the shockwave travel time (link length divided by shockwave speed) for the downstream link is smaller than the $t_{i}$ for the whole corridor, this means the queue backs up to the upstream link. Therefore, $t_{i}$ for the downstream link is 20 minutes and $t_{i}$ for the upstream link is 10 minutes. Where $t_{i}$ information is not available or using the average delay suffices, equation 5.1 can be used for $v / c>1$. For $v / c<1$, the calibrated BPR function is suggested. Therefore, equation 5.4 can be expressed as:

$$
t=\left\{\begin{array}{cc}
t_{0}\left(1+a\left(\frac{v}{c}\right)^{b}\right) & \frac{v}{c} \leq 1 \\
t_{c}+\phi \times \frac{1}{2} T\left(\frac{v}{c}-1\right) & \frac{v}{c} \geq 1
\end{array}\right.
$$

where all the variables are as defined before.

The proposed formulations can be used for both lane-specific and link level purposes if required traffic parameters are obtained appropriately. However, it is worth mentioning that validating the proposed modifications requires further research. 


\subsection{Probabilistic Modification}

As discussed in section 2.6, capacity is treated as a constant value traditionally. However, as shown in section 4.4, it has rather a stochastic nature with a distribution. Also, as introduced earlier in section 3.5, a quantile function of a probability distribution is the inverse of its cumulative distribution function. In this section, the quantile function of capacity probability distribution will be used as a tool to take into account the probabilistic nature of capacity when modeling the travel time and volume to capacity ratio relationship. Toward this end, the quantile function of capacity probability distribution is replaced with the constant value of capacity in the v/c ratio in the proposed speed-flow formulation in the previous section. Therefore the probabilistic version of the equation 5.9, accounting for vehicle's arrival time at the queue, can be expressed as:

$$
t=\left\{\begin{array}{cc}
t_{0}\left(1+a\left(\frac{v}{F_{c}^{-1}(p)}\right)^{b}\right) & \frac{v}{F_{c}^{-1}(p)} \leq 1 \\
t_{c}+\phi \times t_{i}\left(\frac{v}{F_{c}^{-1}(p)}-1\right) & \frac{v}{F_{c}^{-1}(p)} \geq 1
\end{array}\right.
$$

where $F_{c}^{-1}(p)$ is the quantile function of capacity probability distribution and the rest of variables are as defined before.

Similarly, the probabilistic version of the equation 5.10 is as follows: 


$$
t=\left\{\begin{array}{cc}
t_{0}\left(1+a\left(\frac{v}{F_{c}^{-1}(p)}\right)^{b}\right) & \frac{v}{F_{c}^{-1}(p)} \leq 1 \\
t_{c}+\phi \times \frac{1}{2} T\left(\frac{v}{F_{c}^{-1}(p)}-1\right) & \frac{v}{F_{c}^{-1}(p)} \geq 1
\end{array}\right.
$$

where all the variables are as defined before.

It is worth mentioning that all the variables used in the proposed formulations including $t_{0}, t_{c}, t_{i}$, and $\phi$ are random variables. Bottleneck duration is also a random variable. Table 5.2 provides key statistical descriptors for the bottleneck activation, deactivation, and duration time distributions at link level.

Table 5.2. Statistical description of bottleneck activation, deactivation, and duration time at link level

\begin{tabular}{lccccc}
\hline & min & mean & median & max & stdv \\
\cline { 2 - 6 } & \multicolumn{5}{c}{ Milepost 1.92 } \\
\cline { 2 - 6 } Bottleneck activation & $7: 28: 00$ & $7: 39: 22$ & $7: 39: 40$ & $7: 50: 20$ & $0: 05: 45$ \\
Bottleneck deactivation & $8: 00: 00$ & $8: 26: 06$ & $8: 21: 20$ & $9: 07: 40$ & $0: 21: 17$ \\
Bottleneck duration & $0: 15: 40$ & $0: 46: 44$ & $0: 38: 30$ & $1: 31: 00$ & $0: 20: 54$ \\
& \multicolumn{5}{c}{ Milepost 3.12 } \\
Bottleneck activation & $7: 17: 00$ & $7: 30: 42$ & $7: 30: 50$ & $7: 43: 20$ & $0: 05: 52$ \\
Bottleneck deactivation & $8: 03: 40$ & $8: 33: 40$ & $8: 26: 30$ & $9: 07: 20$ & $0: 20: 40$ \\
Bottleneck duration & $0: 33: 40$ & $1: 02: 58$ & $0: 51: 30$ & $1: 37: 20$ & $0: 19: 20$ \\
\hline
\end{tabular}


The values of $T$ that can be applied to equation 5.12 are bounded by zero and the bottleneck duration time. Hence, Table 5.1 can be used to provide an indication of the appropriate values of $T$.
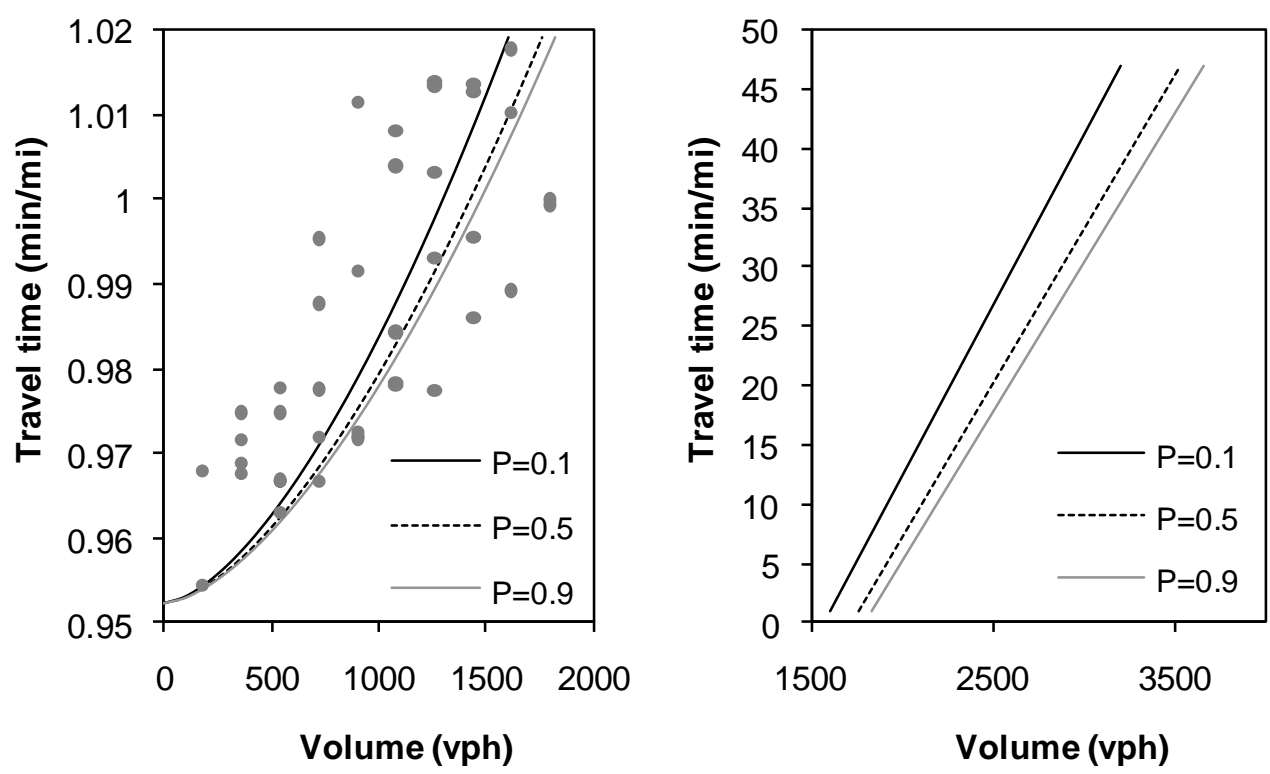

Figure 5.6. Probabilistic volume vs. travel time relationship for the left lane at milepost 1.92 against field data from 5 days

Figure 5.6 shows the volume vs. travel time relationship using equation 5.6 for the left lane at milepost 1.92 where $a=0.07, b=1.6, T=60 \mathrm{~min}, \quad t_{0}=$ $0.9524 \mathrm{~min} / \mathrm{mi}\left(v_{f}=63 \mathrm{mph}\right), t_{c}=1.0190 \mathrm{~min} / \mathrm{mi}\left(v_{c}=58.88 \mathrm{mph}\right)$, and $\quad v_{q}=$ $21.8 \mathrm{mph}(\phi=1.53)$ against field data from 5 days. The empirical quantile function of capacity of the selected lane is used with probabilities of $0.1,0.5$, and 0.9 to plot different curves. 


\subsection{CONCLUSIONS}

This final chapter summarizes the findings of this thesis and proposes possible future work.

\subsection{Summary of Findings}

Different existing speed-flow models for traffic assignment applications were evaluated empirically and theoretically. It was found that for $\mathrm{v} / \mathrm{c}<1$, the calibrated BPR function has the best overall performance among tested models for studied locations. Results showed that for v/c $>1$, the Akçelik and the HCM 2000 models were found to be the most consistent models with queuing theory. It was also found that different freeway lanes and segments may have substantially different traffic characteristics and parameters such as free-flow speed, speed at capacity, and capacity. Therefore, the approach of using speed-flow relationship on individual lanes can be beneficial because it makes it possible to model freeway segments at which the capacity for different reasons corresponds to fraction of lanes.

Results also showed that capacity is a probabilistic measure rather than a constant value. Thus, it is worthwhile to apply an alternative definition of capacity in traffic assignment in light of the probabilistic nature observed. 
Based on the results found, some modifications to speed-flow relationships were proposed. A calibrated probabilistic BPR function was suggested for $\mathrm{v} / \mathrm{c}<1$. While for $\mathrm{v} / \mathrm{c}>1$, a probabilistic speed-flow relationship based on new concepts of queuing theory were proposed. A probabilistic time dependent speed-flow relationship was also proposed for $\mathrm{v} / \mathrm{c}>1$ to account for the vehicle's arrival time at the queue. The probabilistic modification proposed can be used in any v/c ratio based speed-flow model and is not only limited to the proposed speed-flow relationship.

\subsection{Future Work}

There are several research directions in which the ideas presented in this thesis can be continued. The empirical evaluation presented in this thesis used field data only for $\mathrm{v} / \mathrm{c}<1$ conditions and a theoretical approach was taken for $\mathrm{v} / \mathrm{c}>1$. Field data for congested conditions can be obtained from appropriate sources to empirically evaluate the speed-flow models for v/c $>1$. Note that the data must include demand flow and not detected flow. Therefore, data from probe vehicles, vehicle trajectories, or series of loop detectors can be used. Also, simulation is helpful when real field data are not available. Further research is required to validate the proposed modifications. 
In this thesis, only two locations (including four lanes) were studied. To better understand the effects of freeway lane and segment characteristics on traffic measures, more locations from different sites can be studied. Further study should be done on differences between lane-specific and full roadway speed-flow relationships.

This thesis used empirical cumulative distribution of capacity. Using statistical tests such as Kolmogorov-Smirnov test, the observed distributions can be assessed to find out whether a known statistical distribution (e.g. normal, exponential, and etc) can fit the data. Then, when empirical distributions are not available, the empirical quantile function used in the probabilistically modified model can be replaced by the quantile function of the statistical distribution.

Also appropriate mathematical methods such as coordinate transformation method can be used to combine the proposed two equations to develop a single-equation relationship. Also, it will be necessary to test proposed speed-flow relationship in different traffic assignment programs to evaluate the performance of the proposed formulation. 


\subsection{REFERENCES}

Agarwal, M., R.R. Souleyrette, and T. H. Maze (2006). The weather and its impact on urban freeway traffic operations. In Proceedings of the 85nd annual meeting of the Transportation Research Board, Washington, D.C.

Agyemang-Duah K. and F.L. Hall (1991). Some issues regarding the numerical value of capacity. In the Proceedings of International Symposium on Highway Capacity, pp. 1-15, Balkema, Germany.

Ahn, S., R.L. Bertini, B. Auffray, J. Ross (2007). Evaluating the Benefits of a System-Wide Adaptive Ramp-Metering Strategy in Portland, Oregon. Transportation Research Record No. 2012, TRB, National Research Council, Washington, D.C., pp. 47-56.

Akçelik, R. (1991). Travel Time Functions for Transport Planning Purposes: Davidson's Function, its Time-Dependent Form and an Alternative Travel Time Function. Australian Road Research, vol. 21(3), pp 49-59.

Banks J.H. (1990). Flow processes at a freeway bottleneck. Transportation Research Record No. 1287, TRB, National Research Council, Washington, D.C., pp. 20-28.

Banks, J. H. (1991). The Two-Capacity Phenomenon: Some Theoretical Issues. Transportation Research Record No. 1320, TRB, National Research Council, Washington, D.C., pp. 234-241.

Ben-Akiva, M., M. Bierlaire, H. Koutsopoulos, R. Mishalani. (1998) DynaMIT: a simulation-based system for traffic prediction. Presented at the DACCORD Short Term Forecasting Workshop, Delft, Netherlands.

Bertini, R.L., S. Hansen, A. Byrd, and T. Yin (2005). Experience implementing a user service for archived intelligent transportation systems data. In the Transportation Research Record No. 1917, TRB, National Research Council, Washington D.C., pp. 90-99. 
Branston, D. (1976). Link Capacity Functions: A Review. Transportation Research, Vol. 10, pp. 223-236.

Brilon, W., J. Geistefeldt, H. Zurlinden (2007). Implementing the Concept of Reliability for Highway Capacity Analysis. Transportation Research Record No. 2027, TRB, National Research Council, Washington D.C., pp. 1-8.

Carey, M. (1986). A Constraint Qualification for a Dynamic Traffic Assignment Model. Transportation Science, Vol. 20, pp. 55-58.

Carey, M. (1987). Optimal Time Varying Flows on Congested Networks. Operations Research, Vol. 35, No. 1, pp. 58-69.

Cassidy, M.J. and J.R. Windover (1995). Methodology for assessing the dynamics of freeway traffic flow. Transportation Research Record, TRB, National Research Council, Washington D.C., pp. 73-79.

Cassidy M.J. and R.L. Bertini (1999). Some traffic features at freeway bottlenecks. Transportation Research Part B, Vol. 33, pp. 25-42.

Cassidy, M. J. and M. Mauch (2001). An Observed Traffic Pattern in Long Freeway Queues. Transportation Research Part A, Vol. 35, pp.143-156

Chen, H. and C.F. Hsueh (1998). A Model and An Algorithm for the Dynamic User-Optimal Route Choice Problem. Transportation Research Part B, Vol. 32, pp. 219-234.

Chen, C., A. Skabardonis and P. Varaiya (2004). Systematic Identification of Freeway Bottleneck. In the Proceedings of 83rd Transportation Research Board Annual Meeting, Washington, D.C.

Daganzo, C.F. (1994). The cell transmission model: a simple dynamic representation of highway traffic. Transportation Research Part B, Vol. 28, pp. 269-287.

Daganzo, C.F. (2007). Fundamentals of Transportation and Traffic Operations, Emerald Group Publishing Limited. 
Davidson, K.B. (1966). A Flow-Travel Time Relationship for Use in Transportation Planning. In the proceedings of the 3rd ARRB Conference, Vol. 3, No. 1, pp. 183-194.

Davidson, K.B (1978). The theoretical basis of a flow-travel time relationship for use in transportation planning. Australian Road Research, Vol. 8, No. 1, pp. 32-35.

Dong, J. and H.S. Mahmassani (2009). Flow Breakdown and Travel Time Reliability. In the proceedings of 88th Transportation Research Board Annual Meeting, Washington, D.C.

Dowling, R., and A. Skabardonis (1993). Improving the Average Travel Speeds Estimated by Planning Models. In Transportation Research Record No. 1360. TRB, National Research Council, Washington D.C., pp. 68-74.

Dowling, R. G., W. Kittelson, A. Skabardonis, and J. Zegeer (1997). NCHRP Report 387: Techniques for Estimating Speed and Service Volumes for Planning Applications, TRB, National Research Council, Washington, D.C.

Dowling, R., A. Skabardonis, M. Carroll, Z. Wang (2004). Methodology for Measuring Recurrent and Non-recurrent Traffic Congestion. Transportation Research Record 1867, TRB, National Research Council, Washington, D.C., pp. 60-68.

Dowling, R.G. and A. Skabardonis (2008). Urban Arterial Speed-Flow Equations for Travel Demand Models. Innovations in Travel Demand Modeling Conference Proceedings 42, Transportation Research Board, Washington, D.C., Vol. 2, pp. 109-113.

Elefteriadou, L, R.P. Roess, W.R. McShane (1995). Probabilistic nature of breakdown at freeway merge junctions. Transportation Research Record No. 1484, TRB, National Research Council, Washington, D.C., pp. 80-89.

Evans, J. L., L. Elefteriadou, N. Gautam (2001). Probability of breakdown at freeway merges using Markov chains. Transportation Research Part B, Vo. 35, pp. 237-254. 
Geistefelft, J. (2008). Empirical Relation Between Stochastic Capacities and Capacities Obtained from the Speed-Flow Diagram. Presented at the Symposium on the Fundamental Diagram: 75 years, Woods Hole, Massachusetts.

Golding, S. (1978). On Davidson's Flow-Travel Time Relationship for Use in Transportation Planning. Australian Road Research, Vol. 8, No. 3, pp. 36-37.

Hansen, S., A. Byrd, A. Delcambre, A. Rodriguez, S., R.L. Bertini (2005). Using Archived ITS Data to Improve Regional Performance Measurement and Travel Demand Forecasting, 2005 CITE Quad/WCTA Regional Conference Vancouver, BC, Canada.

Highway Capacity Manual (1965). TRB, National Research Council, Washington, D.C.

Highway Capacity Manual (2000). TRB, National Research Council, Washington, D.C.

Hurdle, V.F., M. I. Merlo, D. Robertson (1997). Study of Speed-Flow Relationships on Individual Freeway Lanes. Transportation Research Record No. 1591, TRB, National Research Council, Washington, D.C., pp. 7-13.

Janson, B.N. (1991). Convergent Algorithm for Dynamic Traffic Assignment. Transportation Research Record No. 1328, TRB, National Research Council, Washington, D.C., pp. 69-80.

Kerner, B. S. (2000). Theory of breakdown phenomenon at highway bottlenecks. Transportation Research Record No. 1710, TRB, National Research Council, Washington, D.C., pp. 136-144.

Kerner, B. S., H. Rehborn, M. Aleksic, A. Haug (2004). Recognition and Tracing of Spatial-Temporal Congested Traffic Patterns on Freeways. Transportation Research Part C, Vol. 12, pp.369-400.

Kurth, D.L., A. van Den Hout, B. Ives (1996). Implementation of Highway Capacity Manual-Based Volume-Delay Functions in Regional Traffic 
Assignment Process. Transportation Research Record No. 1556, TRB, National Research Council, Washington, D.C., pp. 27-36.

Kyte, M., Z. Khatib, P. Shannon, and F. Kitchener (2001). Effect of weather on free-flow speed. Transportation Research Record: Journal of the Transportation Research Board, No. 1776, National Research Council, Washington D.C., pp. 60-68.

Li, H., R.L. Bertini (2010). A Comparison of Algorithms for Systematically Tracking Congested Traffic Patterns on Freeways in Portland, Oregon. In the Proceedings of 89th Annual Meeting of the Transportation Research Board, Washington, D.C.

Lindgren, R., R.L. Bertini, D. Helbing, M. Schönhof (2006). Toward Demonstrating the Predictability of Bottleneck Activation on a German Autobahn. Transportation Research Record No. 1965, TRB, National Research Council, Washington, D.C., pp. 12-22.

Lorenz, M.R., and L. Elefteriadou (2001). Defining Freeway Capacity as Function of Breakdown Probability. Transportation Research Record No. 1776. TRB, National Research Council, Washington D.C., pp. 43-51.

Mahmassani, H. S. (2001). Dynamic Network Traffic Assignment and Simulation Methodology for Advanced System Management Applications. Networks and Spatial Economics, Vol. 1, No. 2, pp. 267-292.

May, A.D. (1990). Traffic Flow Fundamentals. Prentice-Hall Inc.

Merchant, DK. and G.L. Nemhauser. (1978) A Model and An Algorthm for the Dynamic Traffic Assignment Problems. Transportation Science, Vol. 12, No. 3, pp. 183-199.

Merchant, D.K. and G.L. Nemhauser. (1978) Optimality Conditions for a Dynamic Traffic Assignment Model. Transportation Science, Vol. 12, No. 3, pp. 200-207. 
Minderhoud, M.M., H. Botma, P.H.L. Bovy (1997). Assessment of Roadway Capacity Estimation Methods. Transportation Research Record No. 1572, TRB, National Research Council, Washington D.C., pp. 59-67.

Munoz, J.C. and C. F. Daganzo (2002). The bottleneck mechanism of a freeway diverge. Transportation Research Part A: Policy and Practice, Vol. 36, pp.483505.

O'Leath, J. L. (1998). Determination of the Probability of Breakdown on a Freeway Based on Zonal Merging Probabilities. M.S. thesis. Department of Civil and Environmental Engineering, Pennsylvania State University, State College.

Olstam, J.J., C. Rydergren, P. Matstoms (2008). Estimation of volume delay functions for urban environments based on an analytical intersection model. In the Proceedings of 87th Transportation Research Board Annual Meeting, Washington, D.C.

Ortuzar, J. De D. and L.G. Willumsen (2001). Modelling Transport, John Wiley \& Sons.

Persaud, B., S. Yagar, R. Brownlee (1998). Exploration of the Breakdown Phenomenon in Freeway Traffic. Transportation Research Record No. 1634. TRB, National Research Council, Washington D.C., pp. 64-69.

Rakha, H., M. Farzaneh, M. Arafeh, and E. Sterzin (2008). Inclement Weather Impacts on Freeway Traffic Stream Behavior. Transportation Research Record: Journal of the Transportation Research Board, No. 2071, National Research Council, Washington D.C., pp. 8-18.

Ran, B., R.W. Hall, D.E. Boyce. (1996) A Link-based Variational Inequality Model for Dynamic Departure Time/Route Choice. Transportation Research Part B, Vol. 30, No. 1, pp. 31-46.

Saberi, M. and R.L. Bertini (2010). Empirical Analysis of the Effects of Rain on Measured Freeway Traffic Parameters. Proceedings of the 89th Annual Meeting of the Transportation Research Board, Washington, D.C. 
Singh, R (1995). Beyond the BPR Curve: Updating Speed-Flow and SpeedCapacity Relationships in Traffic Assignment. Presented at 5th Conference on Transportation Planning Methods Applications, Seattle, Washington.

Singh, R (1999). Improved Speed-Flow Relationships: Application to Transportation Planning Models. Presented at the 7th TRB Conference on Application of Transportation Planning Methods, Boston, Massachusetts.

Skabardonis, A. and R. G. Dowling (1997). Improved Speed-Flow Relationships for Planning Applications. Transportation Research Record No. 1572, TRB, National Research Council, Washington, D.C., pp. 18-23.

Special Report 209: Highway Capacity Manual (1985 and 1994). TRB, National Research Council, Washington, D.C.

Spiess, H. (1990). Conical Volume-Delay Functions. In Transportation Science, Vol. 24, No. 2, 1990.

Tarko, A. and Z. Tian (2003). Example Analysis and Handling of Uncertainty in HCM with Consideration of Traffic Diversion. Transportation Research Record No. 1852, TRB, National Research Council, Washington, D.C., pp. 4046.

Taylor, M.A.P. (1984). A Note on Using Davidson's Function in Equilibrium Assignment. In Transportation Research Part B, Vol. 18, No. 3, pp. 181-199.

Traffic Assignment Manual (1964). Bureau of Public Roads, U.S. Department of Commerce.

Transportation Research Board (1975). Traffic Flow Theory: A Monograph. Special Report 165, National Research Council, Washington, D.C.

Tu, H., H. van Lint, H. J. van Zuylen (2007). Impact of Traffic Flow on Travel Time Variability of Freeway Corridors. Transportation Research Record No. 1993, TRB, National Research Council, Washington, D.C., pp. 59-66. 
Viti, F. and H. J. van Zuylen (2010). Probabilistic models for queues at fixed control signals. Transportation Research Part B, Vol. 44, pp. 120-135.

Waller, S.T., J.L. Schofer, A.K. Ziliaskopoulos (2001). Evaluation with Traffic Assignment Under Demand Uncertainty. Transportation Research Record No. 1771, TRB, National Research Council, Washington, D.C., pp. 69-74.

Wieczorek, J., R. Fernández-Moctezuma, R.L. Bertini (2010). Techniques for Validating an Automatic Bottleneck Detection Tool Using Archived Freeway Sensor Data. In the Proceedings of 89th Annual Meeting of the Transportation Research Board, Washington, D.C.

Zhang L. and D. Levinson (2010). Ramp Metering and Freeway Bottleneck Capacity. Transportation Research Part A, Vol. 44, pp. 218-235. 


\section{APPENDIX A: DAILY CAPACITY AND FREE-FLOW SPEED}

Tables A.1 to A.4 show a list of selected days with their measured free-

flow speed and capacity for each location for each day.

Table A.1. Measured capacity and free-flow speed for left lane at milepost 1.92 on each day.

\begin{tabular}{|c|c|c|c|}
\hline \multirow{4}{*}{ Days } & \multicolumn{3}{|c|}{ Left Lane } \\
\hline & \multicolumn{2}{|c|}{ Capacity } & \multirow{2}{*}{ Free-Flow Speed } \\
\hline & Maximum Flow & Breakdown Flow & \\
\hline & veh/h & veh/h & $\mathrm{mph}$ \\
\hline September 3, 2009 & 1,920 & 1,860 & 63 \\
\hline September 10, 2009 & 1,980 & 1,824 & 63 \\
\hline September 17, 2009 & 2,088 & 1,836 & 63 \\
\hline September 21, 2009 & 1,848 & 1,728 & 63 \\
\hline September 24, 2009 & 2,088 & 2,052 & 63 \\
\hline October 1, 2009 & 1,956 & 1,656 & 62 \\
\hline October 5, 2009 & 1,980 & 1,980 & 63 \\
\hline October 6, 2009 & 1,920 & 1,800 & 62 \\
\hline October 7, 2009 & 2,016 & 2,016 & 63 \\
\hline October 8, 2009 & 1,884 & 1,812 & 63 \\
\hline October 9, 2009 & 1,800 & 1,800 & 62 \\
\hline October 12, 2009 & 2,040 & 1,752 & 62 \\
\hline October 15, 2009 & 2,040 & 2,040 & 61 \\
\hline October 20, 2009 & 2,124 & 2,124 & 61 \\
\hline October 30, 2009 & 1,932 & 1,764 & 62 \\
\hline November 4, 2009 & 2,004 & 2,004 & 64 \\
\hline November 10, 2009 & 1,824 & 1,824 & 62 \\
\hline November 12, 2009 & 1,800 & 1,800 & 61 \\
\hline December 3, 2009 & 2,016 & 2,016 & 63 \\
\hline December 7, 2009 & 2,196 & 1,764 & 62 \\
\hline December 10, 2009 & 1,944 & 1,536 & 63 \\
\hline December 14, 2009 & 1,896 & 1,680 & 62 \\
\hline January 6, 2010 & 2,040 & 1,896 & 61 \\
\hline January 14, 2010 & 1,872 & 1,848 & 61 \\
\hline January 20, 2010 & 2,088 & 1,728 & 62 \\
\hline January 22, 2010 & 1,800 & 1,736 & 62 \\
\hline January 25, 2010 & 2,004 & 2,004 & 61 \\
\hline February 17, 2010 & 2,124 & 2,004 & 62 \\
\hline February 18, 2010 & 1,872 & 1,584 & 62 \\
\hline February 19, 2010 & 1,860 & 1,620 & 62 \\
\hline
\end{tabular}


Table A.2. Measured capacity and free-flow speed for right lane at milepost 1.92 on each day.

\begin{tabular}{|c|c|c|c|}
\hline \multirow{4}{*}{ Days } & \multicolumn{3}{|c|}{ Right Lane } \\
\hline & \multicolumn{2}{|c|}{ Capacity } & \multirow{2}{*}{ Free-Flow Speed } \\
\hline & Maximum Flow & Breakdown Flow & \\
\hline & veh/h & veh/h & $\mathrm{mph}$ \\
\hline September 3, 2009 & 1,536 & 1,476 & 60 \\
\hline September 10, 2009 & 1,524 & 1,404 & 59 \\
\hline September 17, 2009 & 1,644 & 1,332 & 58 \\
\hline September 21, 2009 & 1,416 & 1,248 & 60 \\
\hline September 24, 2009 & 1,608 & 1,608 & 58 \\
\hline October 1, 2009 & 1,560 & 1,356 & 59 \\
\hline October 5, 2009 & 1,560 & 1,548 & 58 \\
\hline October 6, 2009 & 1,644 & 1,428 & 58 \\
\hline October 7, 2009 & 1,548 & 1,284 & 59 \\
\hline October 8, 2009 & 1,392 & 1,344 & 60 \\
\hline October 9, 2009 & 1,392 & 1,392 & 60 \\
\hline October 12, 2009 & 1,584 & 1,392 & 58 \\
\hline October 15, 2009 & 1,452 & 1,452 & 59 \\
\hline October 20, 2009 & 1,380 & 1,140 & 57 \\
\hline October 30, 2009 & 1,380 & 1,176 & 57 \\
\hline November 4, 2009 & 1,452 & 1,452 & 59 \\
\hline November 10, 2009 & 1,560 & 1,368 & 58 \\
\hline November 12, 2009 & 1,428 & 1,428 & 58 \\
\hline December 3, 2009 & 1,404 & 1,380 & 58 \\
\hline December 7, 2009 & 1,488 & 1,260 & 59 \\
\hline December 10, 2009 & 1,404 & 1,332 & 58 \\
\hline December 14, 2009 & 1,464 & 1,464 & 57 \\
\hline January 6, 2010 & 1,524 & 1,404 & 55 \\
\hline January 14, 2010 & 1,356 & 1,356 & 57 \\
\hline January 20, 2010 & 1,572 & 1,332 & 58 \\
\hline January 22, 2010 & 1,316 & 1,292 & 59 \\
\hline January 25, 2010 & 1,440 & 1,440 & 57 \\
\hline February 17, 2010 & 1,524 & 1,368 & 58 \\
\hline February 18, 2010 & 1,488 & 1,032 & 58 \\
\hline February 19, 2010 & 1,356 & 1,308 & 59 \\
\hline
\end{tabular}


Table A.3. Measured capacity and free-flow speed for left lane at milepost 3.12 on each day.

\begin{tabular}{|c|c|c|c|}
\hline \multirow{4}{*}{ Days } & \multicolumn{3}{|c|}{ Left Lane } \\
\hline & \multicolumn{2}{|c|}{ Capacity } & \multirow{2}{*}{ Free-Flow Speed } \\
\hline & Maximum Flow & Breakdown Flow & \\
\hline & $\mathrm{veh} / \mathrm{h}$ & veh/h & $\mathrm{mph}$ \\
\hline September 3, 2009 & 2,100 & 2,100 & 63 \\
\hline September 10, 2009 & 2,172 & 2,172 & 62 \\
\hline September 17, 2009 & 2,232 & 2,208 & 62 \\
\hline September 21, 2009 & 2,400 & 2,160 & 63 \\
\hline September 24, 2009 & 2,376 & 2,376 & 63 \\
\hline October 1, 2009 & 2,088 & 2,004 & 62 \\
\hline October 5, 2009 & 2,484 & 2,484 & 62 \\
\hline October 6, 2009 & 2,160 & 2,160 & 63 \\
\hline October 7, 2009 & 2,020 & 2,020 & 63 \\
\hline October 8, 2009 & 2,328 & 2,196 & 63 \\
\hline October 9, 2009 & 2,136 & 1,908 & 61 \\
\hline October 12, 2009 & 2,040 & 2,040 & 61 \\
\hline October 15, 2009 & 2,124 & 2,124 & 62 \\
\hline October 20, 2009 & 2,184 & 2,028 & 63 \\
\hline October 30, 2009 & 1,968 & 1,968 & 62 \\
\hline November 4, 2009 & 2,196 & 2,160 & 63 \\
\hline November 10, 2009 & 2,268 & 1,956 & 62 \\
\hline November 12, 2009 & 2,112 & 1,788 & 62 \\
\hline December 3, 2009 & 2,088 & 2,088 & 63 \\
\hline December 7, 2009 & 2,112 & 2,112 & 63 \\
\hline December 10, 2009 & 2,112 & 2,064 & 63 \\
\hline December 14, 2009 & 2,196 & 2,064 & 62 \\
\hline January 6, 2010 & 2,184 & 2,124 & 63 \\
\hline January 14, 2010 & 2,124 & 1,860 & 62 \\
\hline January 20, 2010 & 2,424 & 2,268 & 63 \\
\hline January 22, 2010 & 2,160 & 1,932 & 62 \\
\hline January 25, 2010 & 2,112 & 1,932 & 61 \\
\hline February 17, 2010 & 2,280 & 2,064 & 63 \\
\hline February 18, 2010 & 2,232 & 2,208 & 62 \\
\hline February 19, 2010 & 2,244 & 1,824 & 63 \\
\hline
\end{tabular}


Table A.4. Measured capacity and free-flow speed for right lane at milepost 3.12 on each day.

\begin{tabular}{|c|c|c|c|}
\hline \multirow{4}{*}{ Days } & \multicolumn{3}{|c|}{ Right Lane } \\
\hline & \multicolumn{2}{|c|}{ Capacity } & \multirow{2}{*}{ Free-Flow Speed } \\
\hline & Maximum Flow & Breakdown Flow & \\
\hline & veh/h & veh/h & $\mathrm{mph}$ \\
\hline September 3, 2009 & 2,136 & 2,136 & 60 \\
\hline September 10, 2009 & 2,052 & 2,052 & 58 \\
\hline September 17, 2009 & 1,980 & 1,980 & 59 \\
\hline September 21, 2009 & 2,088 & 1,944 & 60 \\
\hline September 24, 2009 & 2,124 & 2,124 & 59 \\
\hline October 1, 2009 & 2,088 & 2,004 & 57 \\
\hline October 5, 2009 & 2,112 & 2,112 & 59 \\
\hline October 6, 2009 & 1,968 & 1,968 & 58 \\
\hline October 7, 2009 & 1,920 & 1,920 & 60 \\
\hline October 8, 2009 & 2,004 & 1,896 & 58 \\
\hline October 9, 2009 & 2,136 & 1,896 & 59 \\
\hline October 12, 2009 & 1,452 & 1,452 & 59 \\
\hline October 15, 2009 & 2,064 & 2,064 & 58 \\
\hline October 20, 2009 & 1,860 & 1,704 & 58 \\
\hline October 30, 2009 & 1,572 & 1,572 & 59 \\
\hline November 4, 2009 & 1,872 & 1,824 & 61 \\
\hline November 10, 2009 & 2,064 & 1,980 & 57 \\
\hline November 12, 2009 & 1,872 & 1,788 & 58 \\
\hline December 3, 2009 & 1,752 & 1,740 & 61 \\
\hline December 7, 2009 & 2,028 & 2,028 & 57 \\
\hline December 10, 2009 & 1,932 & 1,908 & 60 \\
\hline December 14, 2009 & 1,896 & 1,812 & 56 \\
\hline January 6, 2010 & 1,968 & 1,812 & 58 \\
\hline January 14, 2010 & 1,764 & 1,764 & 57 \\
\hline January 20, 2010 & 2,052 & 2,052 & 56 \\
\hline January 22, 2010 & 1,884 & 1,560 & 59 \\
\hline January 25, 2010 & 1,848 & 1,848 & 57 \\
\hline February 17, 2010 & 1,860 & 1,716 & 58 \\
\hline February 18, 2010 & 1,824 & 1,824 & 58 \\
\hline February 19, 2010 & 1,944 & 1,800 & 61 \\
\hline
\end{tabular}




\section{APPENDIX B: CAPACITY DISTRIBUTIONS}

Figures B.1 to B.4 illustrate the distribution of capacity at each location.
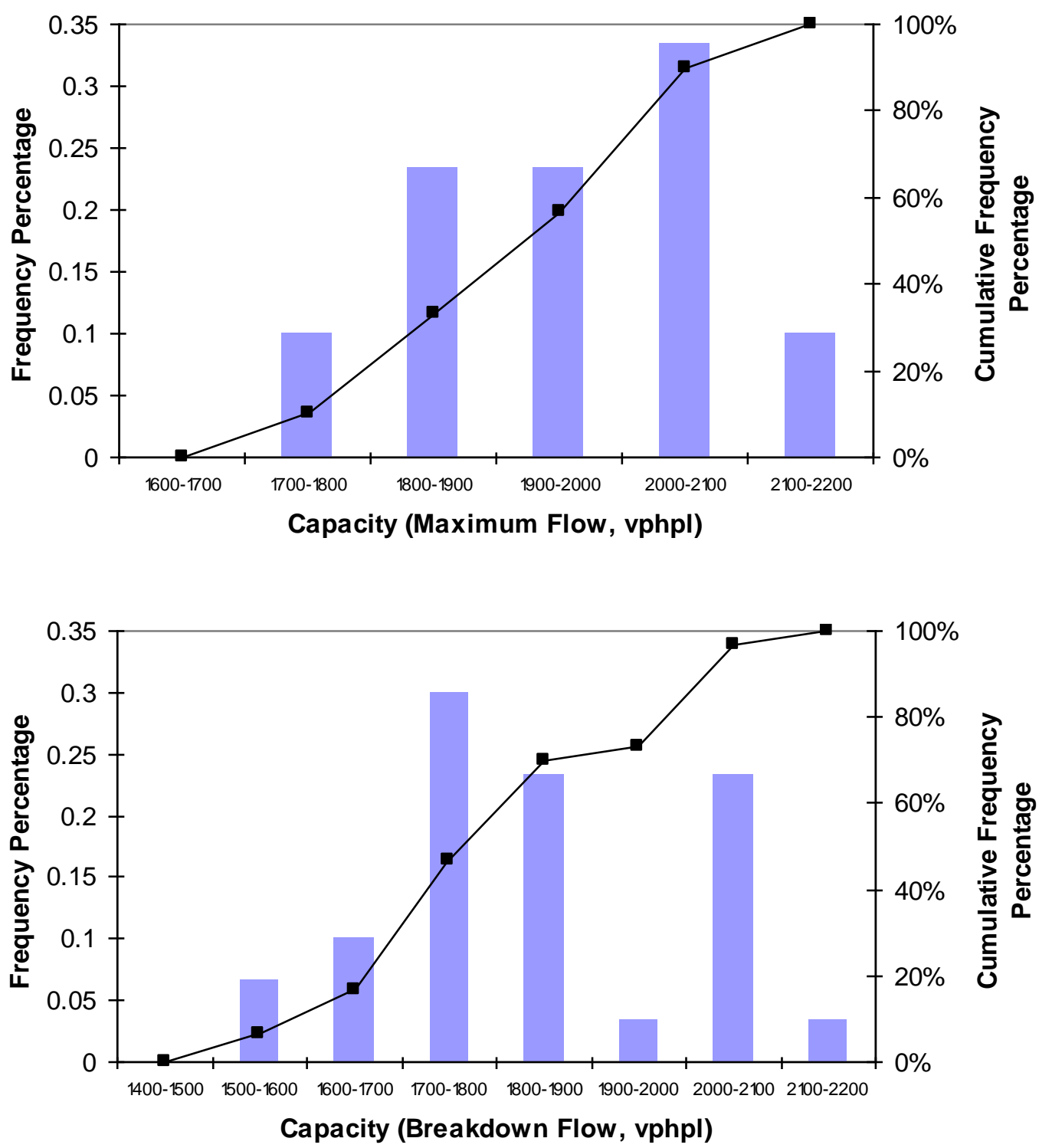

Figure B.1. Capacity distribution for the left lane at milepost 1.92 

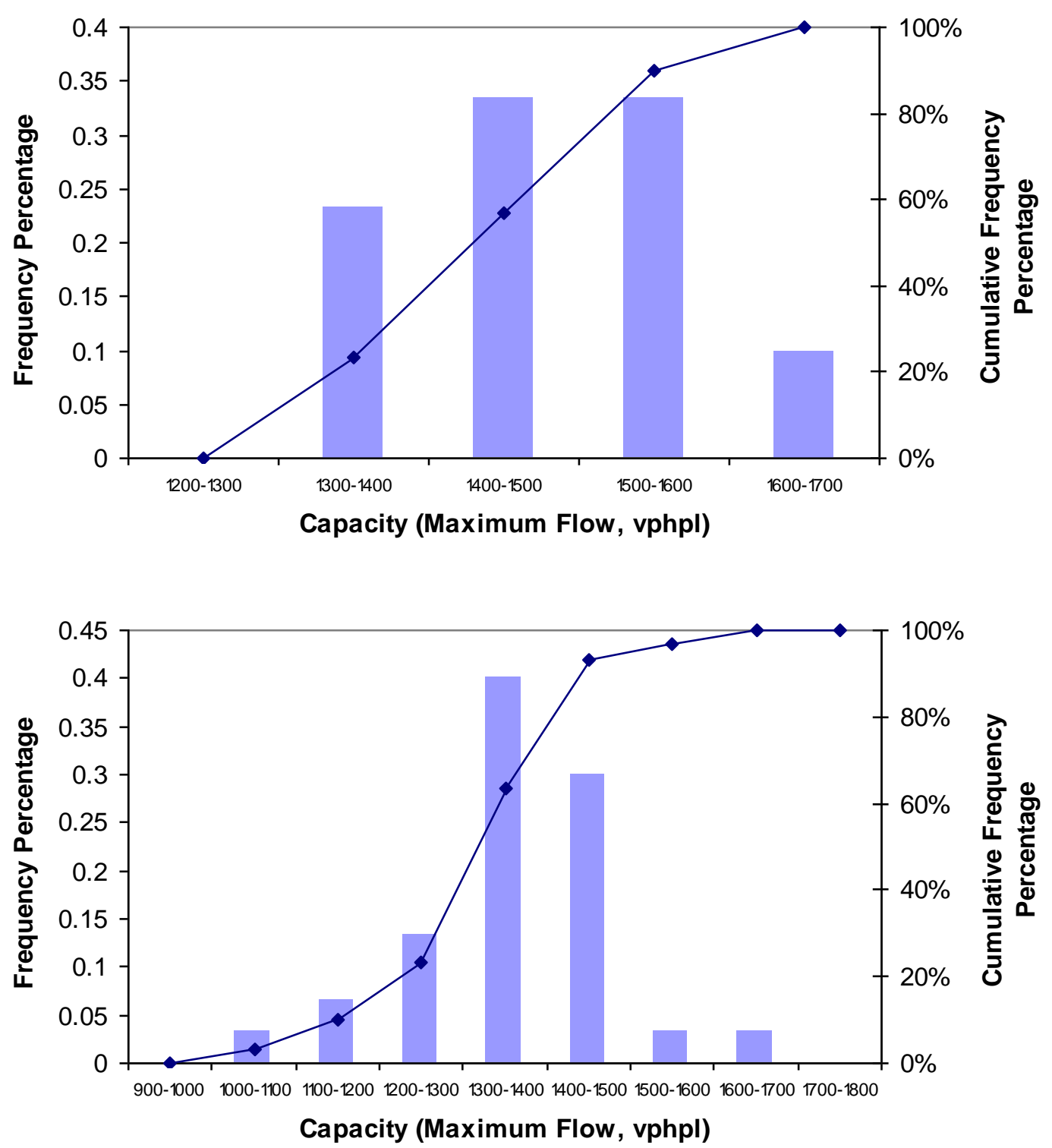

Figure B.2. Capacity distribution for the right lane at milepost 1.92 

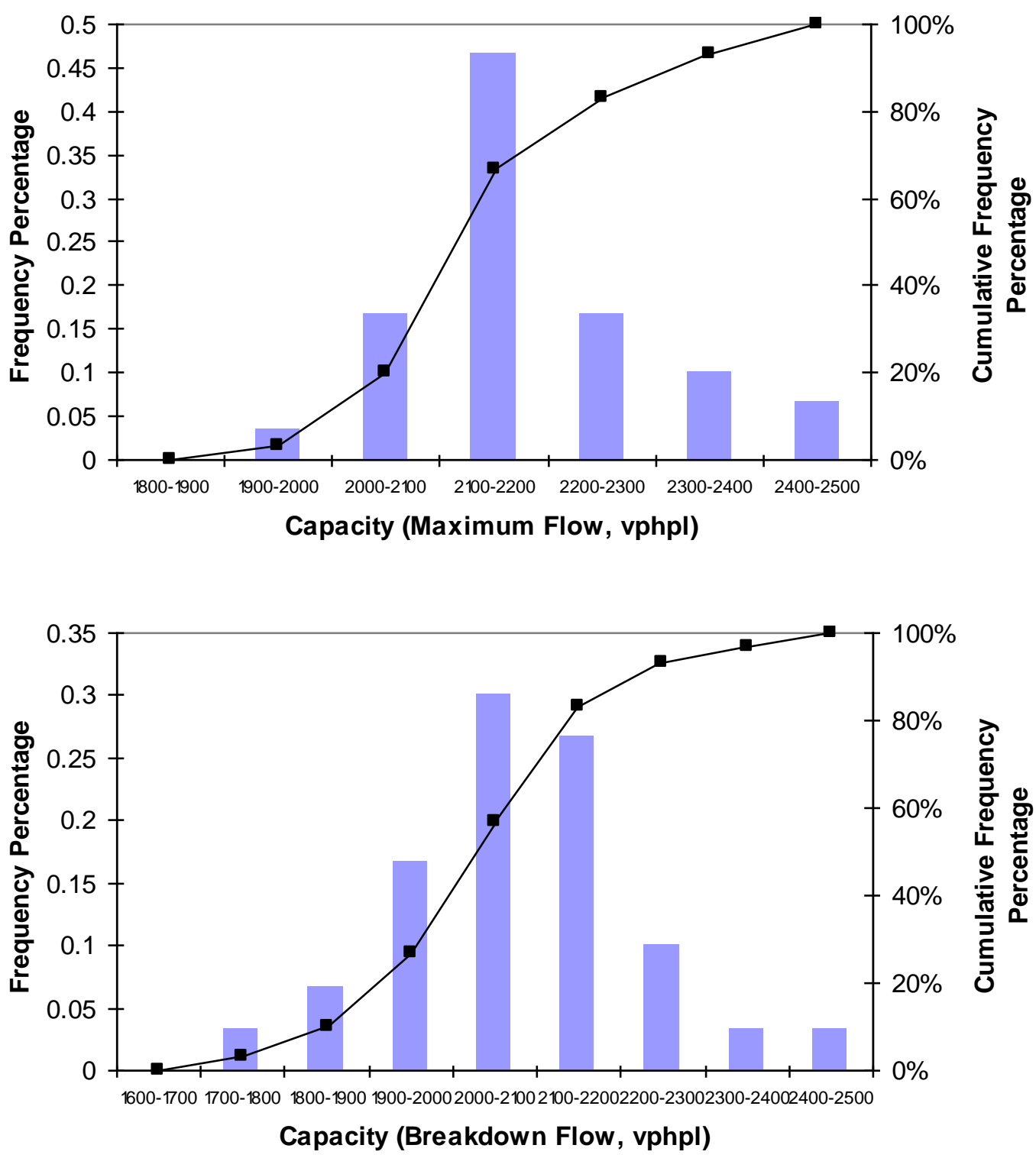

Figure B.3. Capacity distribution for the left lane at milepost 3.12 


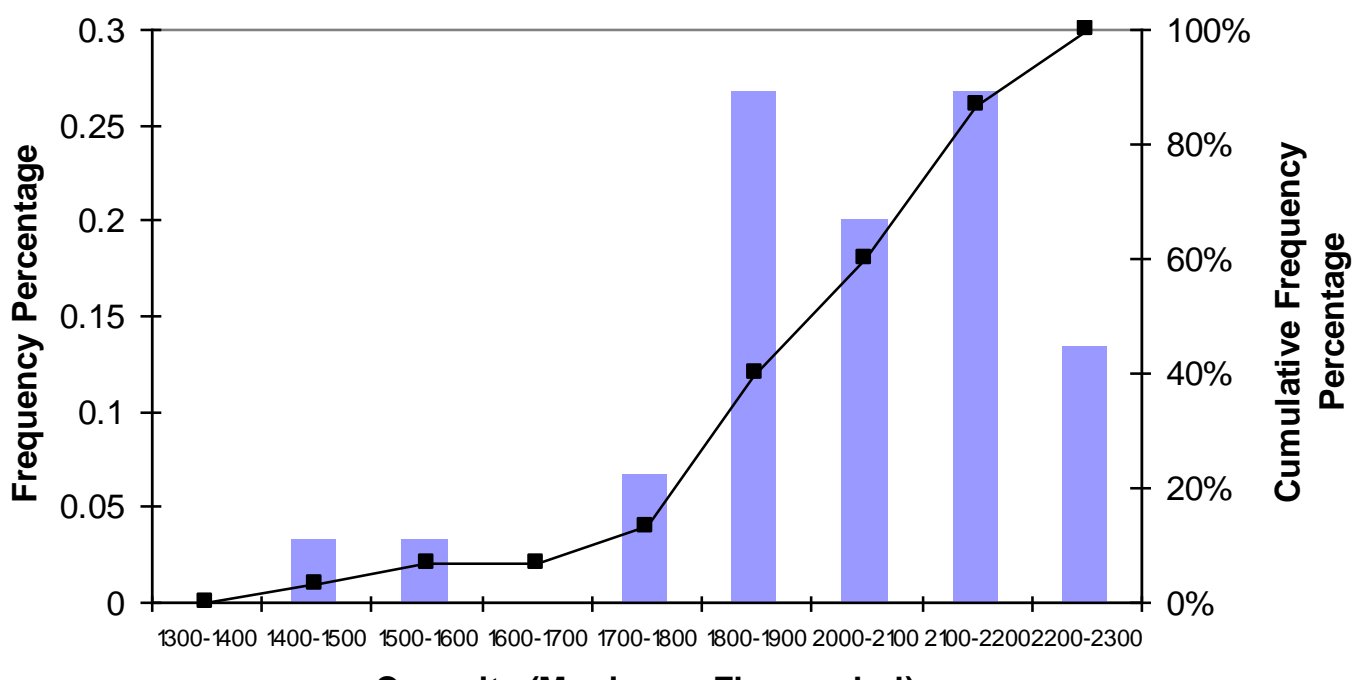

Capacity (Maximum Flow, vphpl)

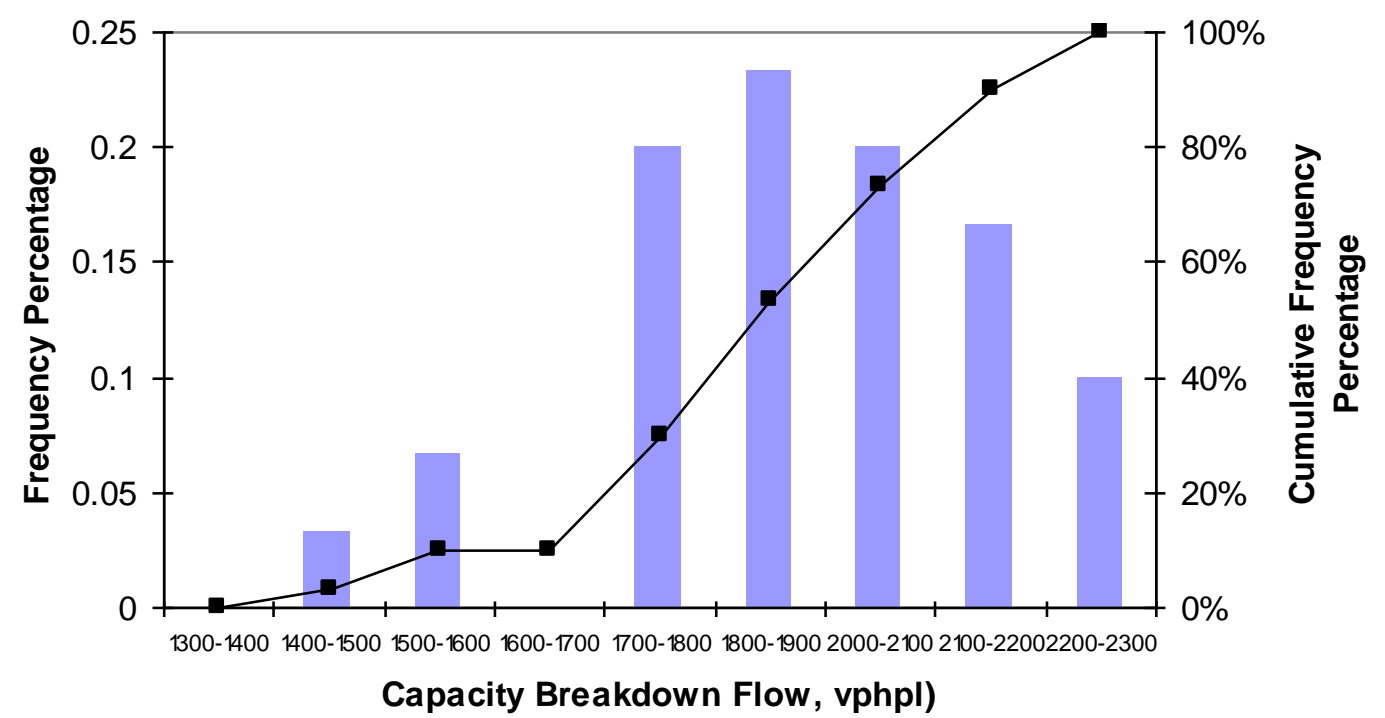

Figure B.4. Capacity distribution for the right lane at milepost 3.12 


\section{APPENDIX C: TRAVEL TIME DISTRIBUTIONS}

Figures C.1 illustrates the distributions of travel time for left lane at milepost 4.35, on January 22th, 2010 plotted in figure 3.7.

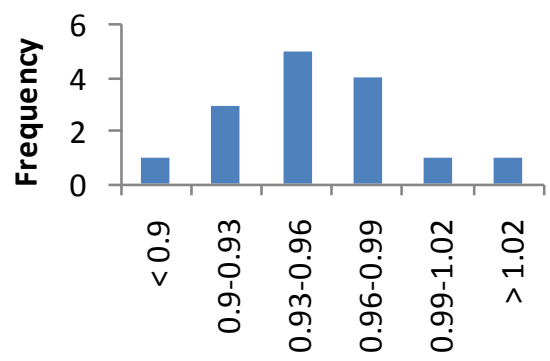

Flow $=180 \mathrm{vph} \quad$ Travel time

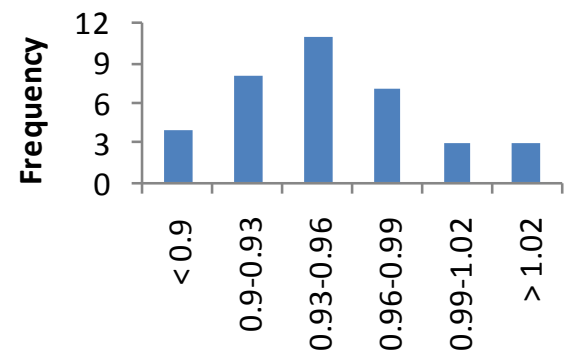

Flow $=540 \mathrm{vph}$

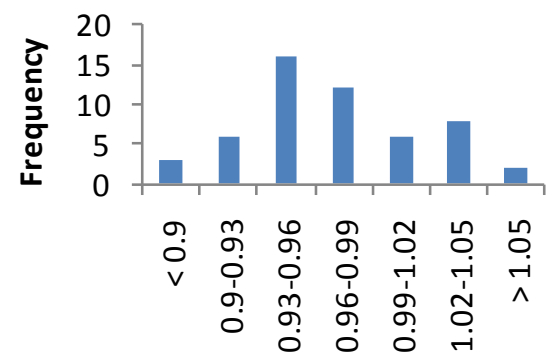

Flow $=900 \mathrm{vph}$
Travel time

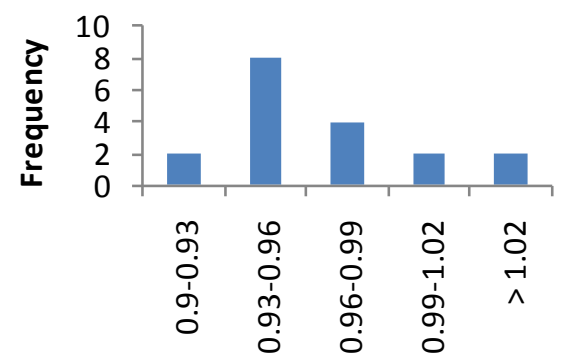

Flow $=360$ vph Travel time

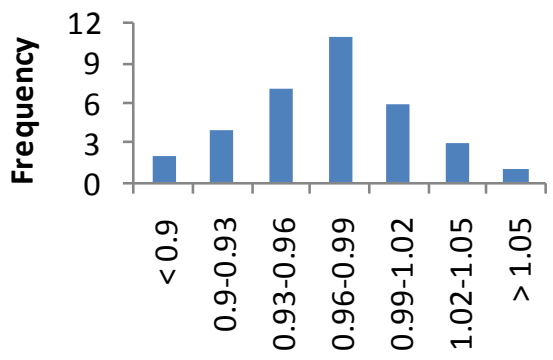

Flow $=720 \mathrm{mph}$

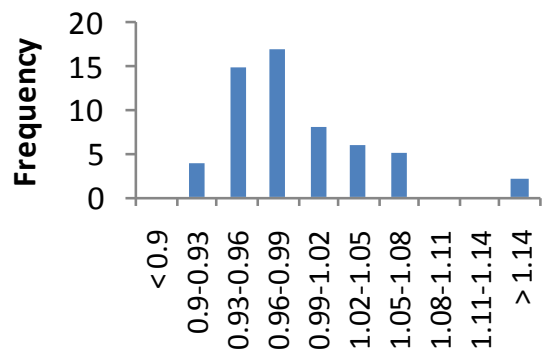

Flow $=1080 \mathrm{vph}$
Travel time

Cont. 


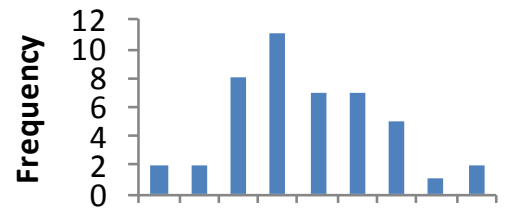

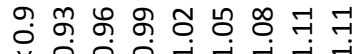

v o 0 o

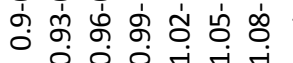

Flow $=1260 \mathrm{vph}$

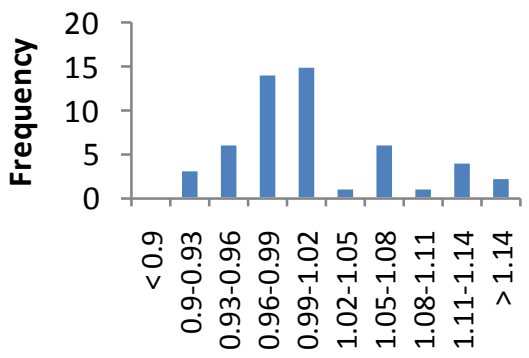

Travel time

Travel time

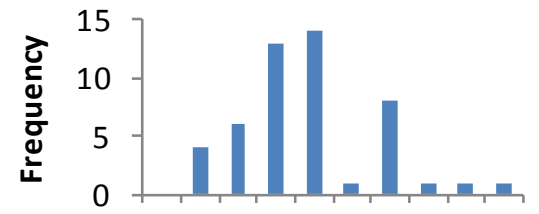

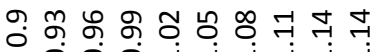

v

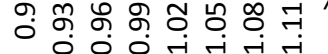

Travel time

Flow $=1620 \mathrm{vph}$

Flow $=1440 \mathrm{vph}$

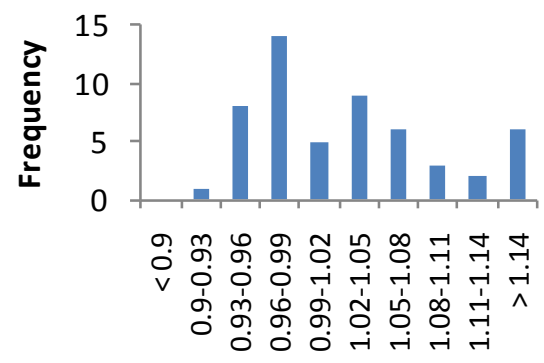

Flow $=1800 \mathrm{vph}$
Travel time

Figure C.1. Travel time distributions for the for left lane at milepost 4.35, on January 22th, 2010 